\title{
Magnetostatic Modeling for Microfluidic Device Design
}

\author{
A Thesis \\ Presented to \\ The Faculty of California Polytechnic State University, \\ San Luis Obispo
}

In Partial Fulfillment

of the Requirements for the Degree

Master of Science in Biomedical Engineering

By

Kirsten Marie Jackson

June 2011 
(C) 2011

Kirsten Marie Jackson

ALL RIGHTS RESERVED 


\section{Committee Approval}

TITLE: Magnetostatic Modeling for Microfluidic Device Design

AUTHOR: Kirsten Marie Jackson

DATE SUBMITTED: June 2011

COMMITTEE CHAIR:

David Clague, Associate Professor of Biomedical Engineering

COMMITTEE MEMBER:

Dan Walsh, Professor of Biomedical Engineering

COMMITTEE MEMBER:

Scott Hazelwood, Associate Professor of Biomedical Engineering 


\section{Abstract}

Magnetostatic Modeling for Microfluidic Device Design

\section{Kirsten Marie Jackson}

For several years, biologists have used superparamagnetic beads to facilitate biological separations. More recently, researchers have adopted this approach in microfluidic devices [1-

3]. This recent development and use of superparamagnetic particles in biomedical and biological applications have resulted in a necessity for methods that enable the understanding and prediction of their properties and actions during use. Typically, such methods would involve simple experimentation prior to in vitro experimentation, animal testing, and finally clinical testing. To better understand and unleash this technology, $\mathrm{COMSOL}^{\circledR}$, which is a finite element analysis and multiphysics simulation software, has recently been used to model superparamagnetic particles in several applications.

Two $\mathrm{COMSOL}^{\circledR}$ models were created based on a magnetic trapping system consisting of a stationary permanent magnet and a microfluidics channel. The first model, known as the Particle Motion Model, simulates the movement of an individual superparamagnetic particle flowing through a fluidic channel beneath a permanent magnet by coupling the Moving Mesh Arbitrary Lagrange-Eulerian (ALE), Incompressible Navier-Stokes, Plane Strain, and Magnetostatics physics modes. The second model, which will be referred to as the Mixture Model, simulates the bulk flow of a superparamagnetic particle fluid in an identical system using the Mixture and Magnetostatics physics modes. The Mixture Model was then applied to the optimization of the design of the magnetic trapping system using a defined geometry and velocity. 


\section{Acknowledgements}

I would like to thank Dr. David Clague for numerous useful discussions of my project. In particular, he helped on the Mixture Model and magnetophoresis. I also appreciate his continual support and encouragement during my project.

Thanks to the Naval Research Laboratory in Washington, DC, for exposing me to experimental applications of microflow cytometry and microfluidic devices.

Finally, I would like to thank my family for always supporting and encouraging me. I would not be where I am without them. 


\section{Table of Contents}

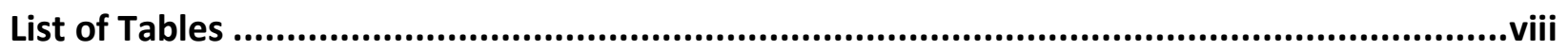

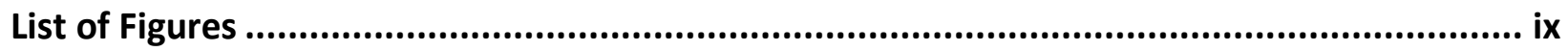

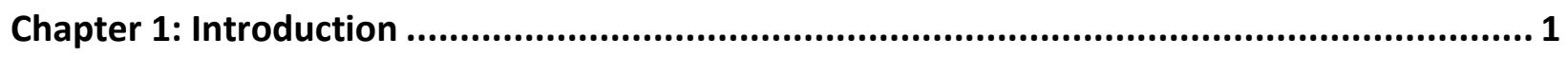

1.1 Drug Delivery.

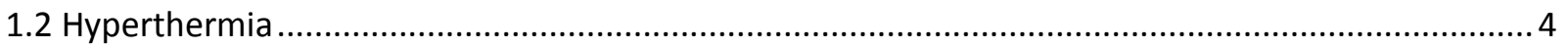

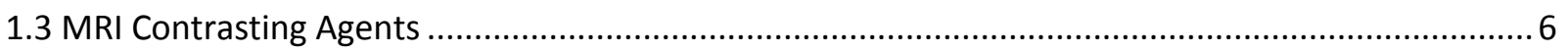

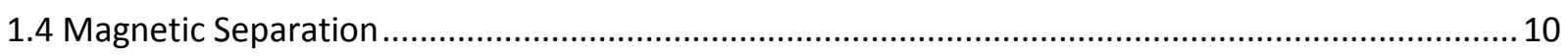

1.5 Modeling of Superparamagnetic Particles................................................................................ 12

1.6 The Magnetic Separator ....................................................................................................... 17

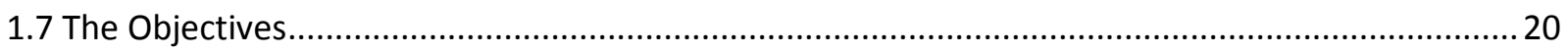

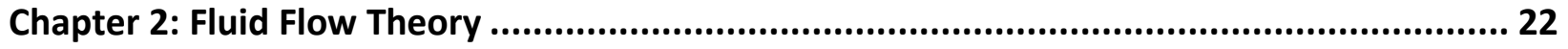

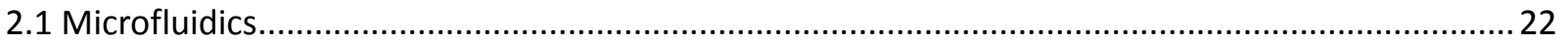

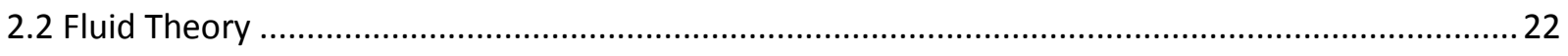

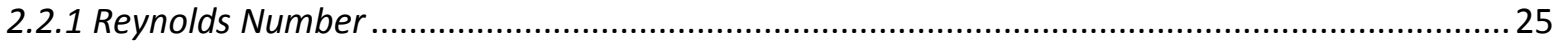

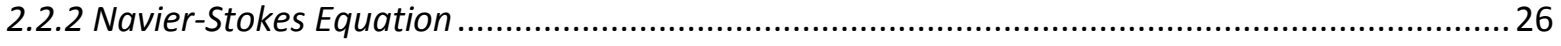

Chapter 3: Magnetic Theory .................................................................................................. 29

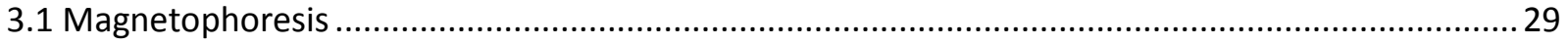

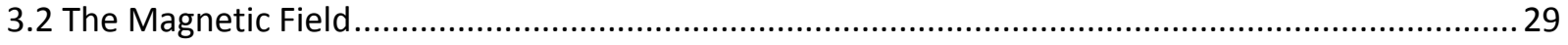

3.3 Magnetic Field Background ................................................................................................... 31

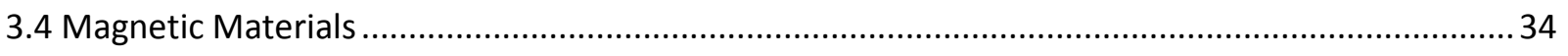

3.4.1 Diamagnetic Materials......................................................................................................

3.4.2 Paramagnetic Materials .............................................................................................

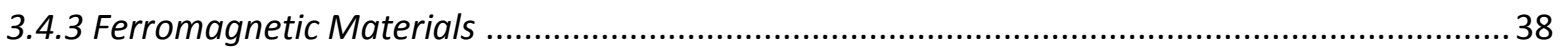

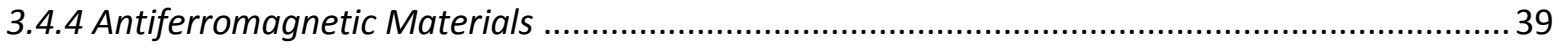

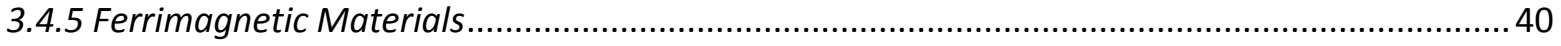

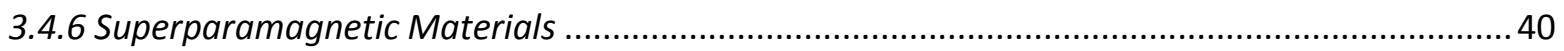

3.5 Magnetophoresis of a Superparamagnetic Bead ……………................................................. 42

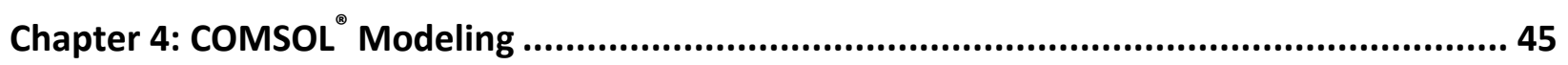

4.1 Implementing the Particle Motion Model ..................................................................................... 45 


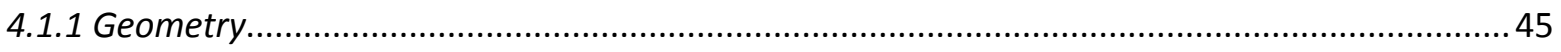

4.1.2 The Moving Mesh ALE Mode ................................................................................................... 46

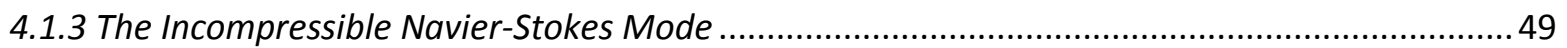

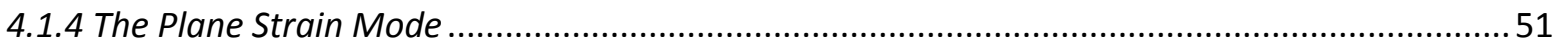

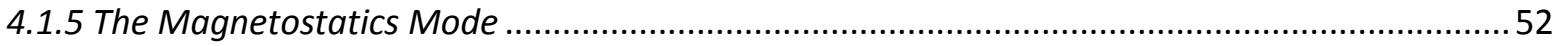

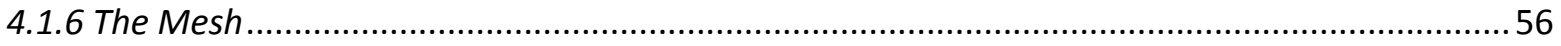

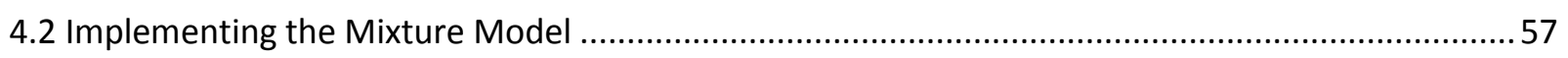

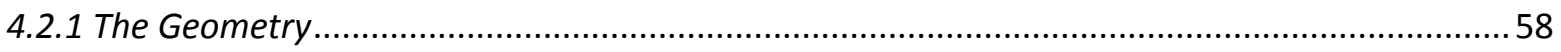

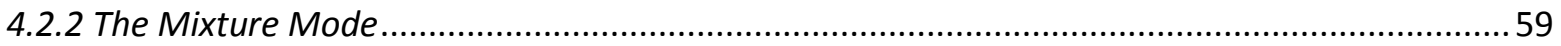

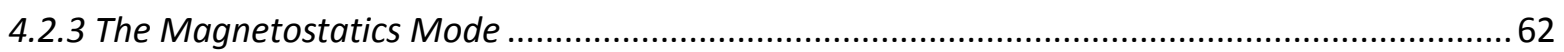

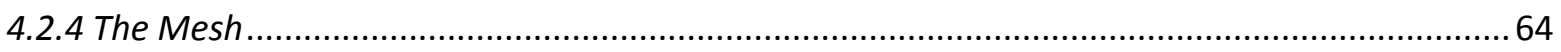

Chapter 5: Modeling Results and Future Directions ................................................... 66

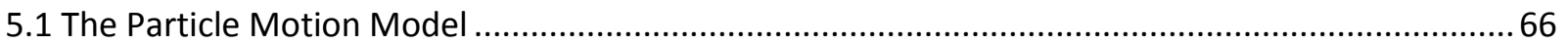

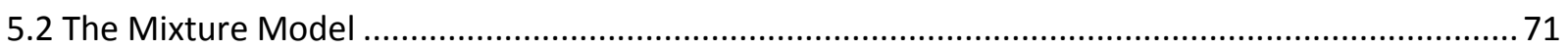

5.2.1 Design Optimization Using the Mixture Model...................................................................... 78

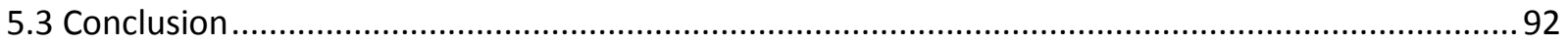

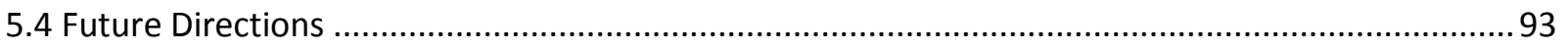

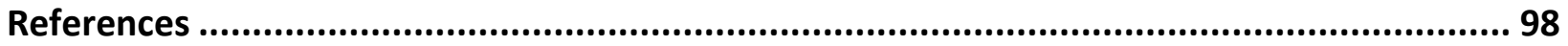

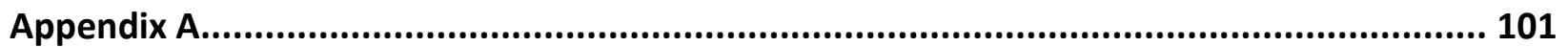

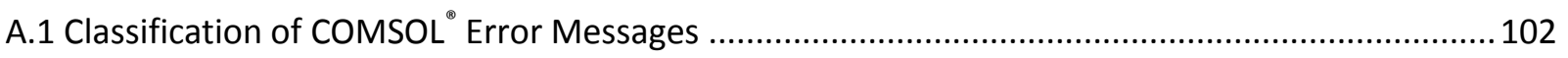

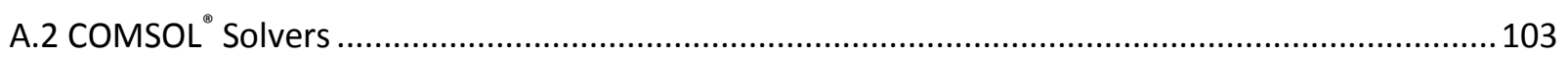

A.3 Linear System Solvers in COMSOL 


\section{List of Tables}

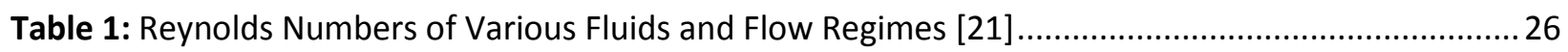

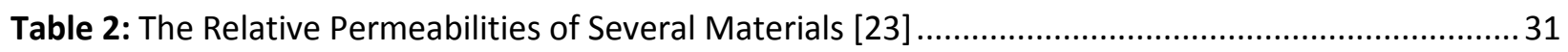

Table 3: The Magnetic Susceptibility of Various Materials [25] ......................................................... 34

Table 4: A Table Summary of the Classification of Magnetic Materials [26].......................................... 36

Table 5: The Variable Definitions for the Forces Exerted on the Particle Motion Model ......................... 52

Table 6: The Volumetric Forces Implemented on the Mixture Model ..................................................60

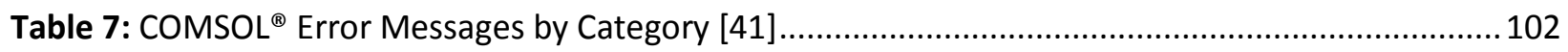

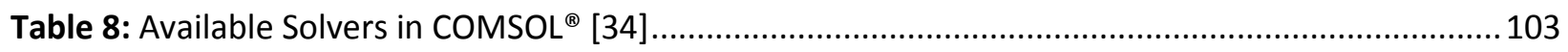

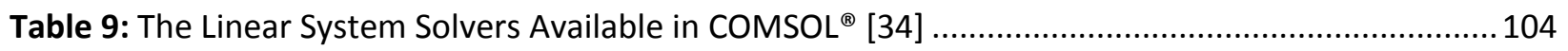




\section{List of Figures}

Figure 1: Representation of a Functionalized Superparamagnetic Particle [3] ...................................... 1

Figure 2: Illustration of Targeted Delivery Using Magnetostatics [3] ................................................... 3

Figure 3: Temperature Results from Hyperthermia Treatment in Rabbits [3] ...................................... 4

Figure 4: Scans of Glioblastoma Treatment Using Magnetic Particles [4] ........................................... 5

Figure 5: Superparamagnetic Particles as an MRI Contrasting Agent in a Rat Lymph Node Tumor

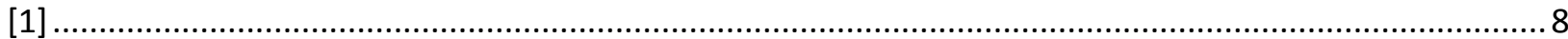

Figure 6: Representation of Functionalized Nanoparticles Used as an MRI Contrasting Agent and Drug Delivery Method [7]

Figure 7: Scans of Cancerous Tissue Before and After the Injection of Functionalized Superparamagnetic Nanoparticles [8]

Figure 8: Possible Magnetic Separator Designs [3]

Figure 9: Micromixer and Magnetic Separator Used for HIV Type 1 Virion Detection [9] 12

Figure 10: $\mathrm{COMSOL}^{\circledR}$ Model of Ferrofluid in Bloodstream [10]

Figure 11: Previous $\mathrm{COMSOL}^{\circledR}$ Modeling of Individual Superparamagnetic Beads [11] ........................ 15

Figure 12: The Magnetic Trapping System Used for Toxic Particle Detection [12] ............................... 16

Figure 13: $\mathrm{COMSOL}^{\circledR}$ Model of Magnetic Trapping System Used for Toxic Particle Detection [12] .......... 17

Figure 14: Graphic Depiction of the Magnetic Trapping System ..................................................... 18

Figure 15: $\mathrm{COMSOL}^{\circledR}$ Modeling of the Magnetic Separator ............................................................... 19

Figure 16: A Larger View of the Microfluidic Channel Beneath the Stationary Magnet ...........................20

Figure 17: The Forces Exerted on a Superparamagnetic Bead in a Microfluidic Channel.........................27

Figure 18: The Magnetic Field of a Permanent Magnet as a Result of Loose Iron Filings [30] .................30

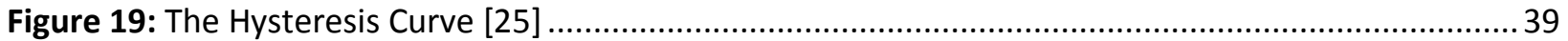

Figure 20: An Illustration of the Alignment of Magnetic Domains in a Magnetic Field [30] ..................... 40

Figure 21: Magnetic Field Intensity Plot for Superparamagnetic Materials [31] .................................. 41 
Figure 22: Magnetic Field Intensity Plot for Superparamagnetic Materials for a Range of -3.5 to $3.5 \mathrm{mT}[31]$.....

Figure 23: The Geometry of the Particle Motion Model Consisting of a Rectangular Magnet, Thin Fluidic Channel, a Small Particle, and Surrounding Air.... 46

Figure 24: Illustration of the Normal Mesh Velocity Boundary Condition 47

Figure 25: An Illustration of the Moving Mesh ALE Mode Properties for the Particle Motion Model

Figure 26: The Particle Boundary Conditions for the Incompressible Navier-Stokes Mode 50

Figure 27: The Magnetostatics Mode Subdomain Settings for the Surrounding Air .53

Figure 28: The Magnetostatics Mode Subdomain Settings for Water in the Particle Motion Model

Figure 29: The Magnetostatics Mode Subdomain Settings for Permanent Magnet in the Particle Motion Model .55

Figure 30: The Magnetostatics Mode Subdomain Settings for the Particle in the Particle Motion Model .56

Figure 31: The Mesh of the Particle Motion Model .57

Figure 32: The Geometry of the Mixture Model Consisting of a Rectangular Magnet, Thin Fluidic Channel, a Small Circular Inlet, and Surrounding Air .58

Figure 33: The Mixture Model Boundary Conditions for the Inlet ..... 61

Figure 34: The Magnetostatics Mode Subdomain Settings for the Channel in the Mixture Model 62

Figure 35: The Magnetostatics Mode Subdomain Settings for the Inlet of the Mixture Model 63

Figure 36: The Mesh for the Mixture Model 64

Figure 37: The Convergence Study for the Mixture Model 65

Figure 38: The Fluid Velocity Surface Plot and Magnetic Potential Contour Plot of the Particle Motion Model.... 66

Figure 39: The Magnetic Potential of the Particle Motion Model. .67

Figure 40: The Magnetic Field of the Particle Motion Model. 68

Figure 41: An Illustration of the Particle's Movement 69

Figure 42: Particle Motion at $0.01 \mathrm{~s}$ (Left) and 0.02s (Right) 70 


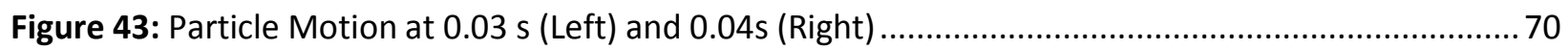

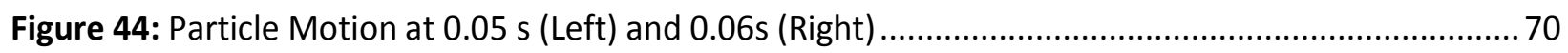

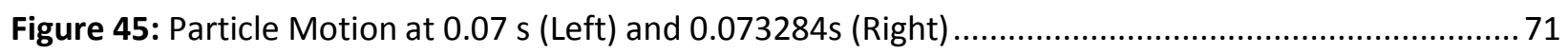

Figure 46: Surface Plot of the Superparamagnetic Fluid Volume Fraction and Magnetic Potential Contour Plot for the Mixture Model at $0.33 \mathrm{~s}$

Figure 47: Superparamagnetic Particle Fluid Movement at $0 \mathrm{~s}$ (Left) and $0.05 \mathrm{~s}$ (Right) for the Mixture Model.....

Figure 48: Superparamagnetic Particle Fluid Movement at $0.10 \mathrm{~s}$ (Left) and $0.15 \mathrm{~s}$ (Right) for the Mixture Model

Figure 49: Superparamagnetic Particle Fluid Movement at $0.20 \mathrm{~s}$ (Left) and $0.25 \mathrm{~s}$ (Right) for the Mixture Model.....

Figure 50: Superparamagnetic Particle Fluid Movement at $0.30 \mathrm{~s}$ (Left) and $0.35 \mathrm{~s}$ (Right) for the Mixture Model .

Figure 51: Superparamagnetic Particle Fluid Movement at $0.40 \mathrm{~s}$ (Left) and $0.45 \mathrm{~s}$ (Right) for the Mixture Model .

Figure 52: Superparamagnetic Particle Fluid Movement at $0.50 \mathrm{~s}$ (Left) and $0.55 \mathrm{~s}$ (Right) for the Mixture Model

Figure 53: Superparamagnetic Particle Fluid Movement at $0.60 \mathrm{~s}$ (Left) and $0.65 \mathrm{~s}$ (Right) for the Mixture Model .....

Figure 54: Superparamagnetic Particle Fluid Movement at $0.70 \mathrm{~s}$ (Left) and $0.75 \mathrm{~s}$ (Right) for the Mixture Model . 75

Figure 55: Superparamagnetic Particle Fluid Movement at $0.80 \mathrm{~s}$ (Left) and $0.85 \mathrm{~s}$ (Right) for the Mixture Model....

Figure 56: Volume Fraction of the Superparamagnetic Particle Fluid beneath the Left Side of the Magnet.

Figure 57: Volume Fraction of the Superparamagnetic Particle Fluid beneath the Middle of the Magnet.

Figure 58: Volume Fraction of the Superparamagnetic Particle Fluid beneath the Right Side of the Magnet.

Figure 59: The Volume Fraction Surface Plot and Magnetic Potential Contour Plot for a Mixture Model that Includes Inertial Effects.

Figure 60: The Volume Fraction Plot for the Inertial Mixture Model beneath the Left Side of the Magnet. 
Figure 61: The Volume Fraction Plot for the Inertial Mixture Model beneath the Middle of the Magnet.

Figure 62: The Volume Fraction Plot for the Inertial Mixture Model beneath the Right Side of the Magnet......

Figure 63: The Volume Fraction Surface Plot and Magnetic Potential Contour Plot of a Mixture Model with Reverse Orientation of the Channel and Magnet

Figure 64: The Volume Fraction Plot for the Reversed Orientation Mixture Model above the Left Side of the Magnet.

Figure 65: The Volume Fraction Plot for the Reversed Orientation Mixture Model above the Middle of the Magnet.

Figure 66: The Volume Fraction Plot for the Reversed Orientation Mixture Model above the Right Side of the Magnet .....

Figure 67: The Volume Fraction Surface Plot and Magnetic Potential Contour Plot for a Reversed Orientation Mixture Model with an Imposed Magnetic Field of $1 \mathrm{~T}$.

Figure 68: The Volume Fraction of the Mixture Model with the Increased Magnetic Field above the Left Side of the Magnet

Figure 69: The Volume Fraction of the Mixture Model with the Increased Magnetic Field above the Middle of the Magnet.

Figure 70: The Volume Fraction of the Mixture Model with the Increased Magnetic Field above the Right Side of the Magnet

Figure 71: The Volume Fraction Surface Plot and Magnetic Potential Contour for a Model with a Decreased Magnetic Field.....

Figure 72: The Volume Fraction of the Mixture Model with the Decreased Magnetic Field above the Left Side of the Magnet.

Figure 73: The Volume Fraction of the Mixture Model with the Decreased Magnetic Field above the Middle of the Magnet.

Figure 74: The Volume Fraction of the Mixture Model with the Decreased Magnetic Field above the Right Side of the Magnet

Figure 75: The Velocity Fields for the Channel Height of the System for Stagnant (Left) and Regular (Right) Flow Conditions for a Magnetic Field Strength of 0.25 T.

Figure 76: The Velocity Fields for the Channel Height of the System for Stagnant (Left) and Regular (Right) Flow Conditions for a Magnetic Field Strength of $0.5 \mathrm{~T}$. 
Figure 77: The Velocity Fields for the Channel Height of the System for Stagnant (Left) and

Regular (Right) Flow Conditions for a Magnetic Field Strength of $1 \mathrm{~T}$.

Figure 78: Particle Motion in the Particle Phase Field Mode at $0 \mathrm{~s}$ (Left) and $0.01 \mathrm{~s}$ (Right) ................... 95

Figure 79: Particle Motion in the Particle Phase Field Mode at $0.02 \mathrm{~s}$ (Left) and $0.03 \mathrm{~s}$ (Right) ............... 96

Figure 80: Particle Motion in the Particle Phase Field Mode at $0.04 \mathrm{~s}$ (Left) and $0.05 \mathrm{~s}$ (Right) ............... 96

Figure 81: Particle Motion in the Particle Phase Field Mode at $0.06 \mathrm{~s}$ (Left) and $0.07 \mathrm{~s}$ (Right) ...............96

Figure 82: Particle Motion in the Particle Phase Field Mode at 0.08 s (Left) and 0.09 s (Right) ............... 97

Figure 83: Particle Motion in the Particle Phase Field Mode at $0.095 \mathrm{~s}$ (Left) and $0.1 \mathrm{~s}$ (Right) .............. 97 


\section{Chapter 1: Introduction}

The field of medical diagnostics is constantly evolving to treat and prevent illnesses and diseases through innovation and technology. One relatively new technique is the application of functionalized superparamagnetic particles to the detection and treatment of diseased tissue. All living organisms are affected by magnetism; for example, a species of bacteria, known as magnetotactic bacteria, orient themselves with the magnetic field of the earth. Even in the human body, hemoglobin has a magnetic nature because of its iron complex [1]. However, in regard to healthcare, magnetism has commonly been used for the extraction of metallic objects within a body, and iron oxides are frequently used as iron supplements [2].

Superparamagnetic particles offer unique properties beneficial to the health care field. Consisting of inorganic ferromagnetic particles surrounded by an organic and dielectric medium, these particles are magnetic in the presence of a magnetic field and can be easily manipulated. For example, an organic medium can be tagged with functionalized particles for targeting, and the size of the functionalized particles can range from a few nanometers to microns, allowing close interaction with cells and other biomolecules. An illustration of a functionalized superparamagnetic particle with a ferrite core, silicon dioxide $\left(\mathrm{SiO}_{2}\right)$ shell, and functional groups represented by the letter $\mathrm{F}$ can be seen in Figure $1[3]$.

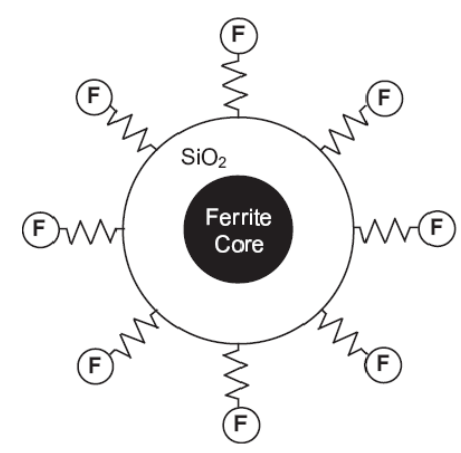

Figure 1: Representation of a Functionalized Superparamagnetic Particle [3] 
As a result of this approach, superparamagnetic particles have been successfully applied to drug delivery, hyperthermia treatment, MRI imaging as a contrasting agent, and magnetic separation. Each application of these particles will be described in this section, but the use of superparamagnetic particles for magnetic separation will be the primary focus of further sections.

\subsection{Drug Delivery}

The most common method of treating cancer involves the introduction of cytotoxic drug therapies through non-specific means such as chemotherapy and radiation therapy, resulting in negative side effects and the exposure of adjacent healthy cells. In addition, the drugs often have to travel through many tissues and cells of the body, lessening the dose that reaches the cancerous cells. Having identified the unique properties and abilities of superparamagnetic nanoparticles, researchers have been investigating their application as a method of targeted drug delivery for cancer treatment since the 1970s. By attaching the cytotoxic drug to the nanoparticles, injecting the nanoparticles, using a magnetic field to concentrate the nanoparticles to a specific site, and releasing the drugs for up-take by the cancerous cells, researchers hope to be able to reduce the side effects and amount of drug exposed to normal and healthy cells. Figure 2 depicts this targeted delivery through the bloodstream; the cytotoxic drug-attached nanoparticles are frequently introduced intravenously as a ferrofluid, or fluidic suspension of superparamagnetic nanoparticles [3]. 


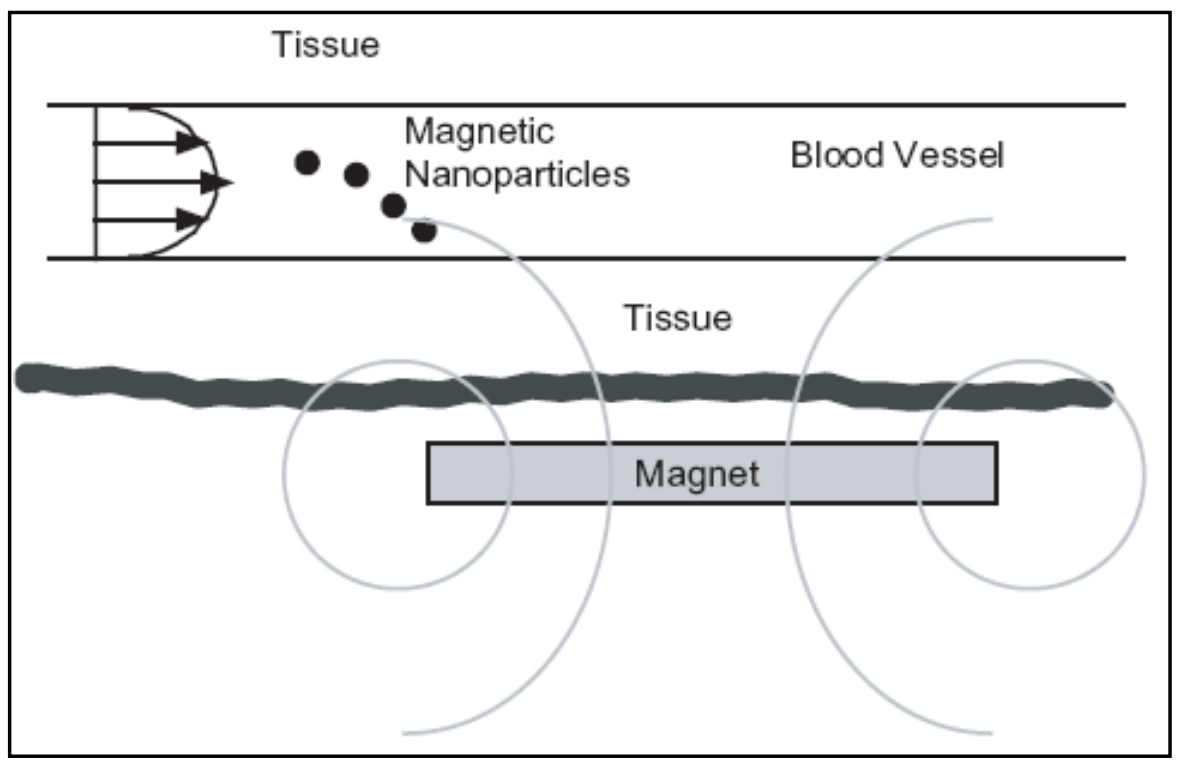

Figure 2: Illustration of Targeted Delivery Using Magnetostatics [3]

Targeted drug delivery has proven to be successful in animal testing. In 1983, targeted doxorubicin delivery was applied to sarcoma tumors in rat tails. The results showed that all rats were in remission following targeted delivery compared to no rats in remission when only the drug was given to the rats. In a similar study, permanent magnets were implanted at osteosarcoma sites of hamsters, and cytotoxic drugs were targeted to the location. As a result, drug delivery increased four-fold over typical intravenous drug targeting, and weight-loss as a side effect was eliminated [3].

The application of targeted drug delivery has many issues in regards to application in humans.

These issues include: the possibility of a blood vessel embolism if nanoparticle accumulation occurs, the difficulty in scaling up for humans, determining a method of nanoparticle removal once drugs are released, and ensuring no toxic effect occurs in response to the nanoparticles. As a result of these challenges, not much clinical testing of targeted drug therapy has occurred, but the available studies have shown successful progress. As of 2002, ferrofluids have been successfully tolerated by 14 people in a hepatocellular carcinoma clinical trial. In addition, this same study was able to direct the ferrofluid toward the targeted area [3]. 


\subsection{Hyperthermia}

Since 1957, researchers have been investigating the use of superparamagnetic particles in exposing diseased cells to hyperthermia conditions to destroy those cells. This endeavor is achieved by injecting the particles into the targeted tissue and applying an AC magnetic field that will cause the particles to selectively heat the diseased tissue to above $42^{\circ} \mathrm{C}$ for over 30 minutes, killing them. Figure 3 shows the achieved temperature results for an animal study in rabbits. As it illustrates, hyperthermia conditions were achieved in the tumor edge and center (top two lines) while sparing any adjacent healthy tissue (bottom three lines) [3].

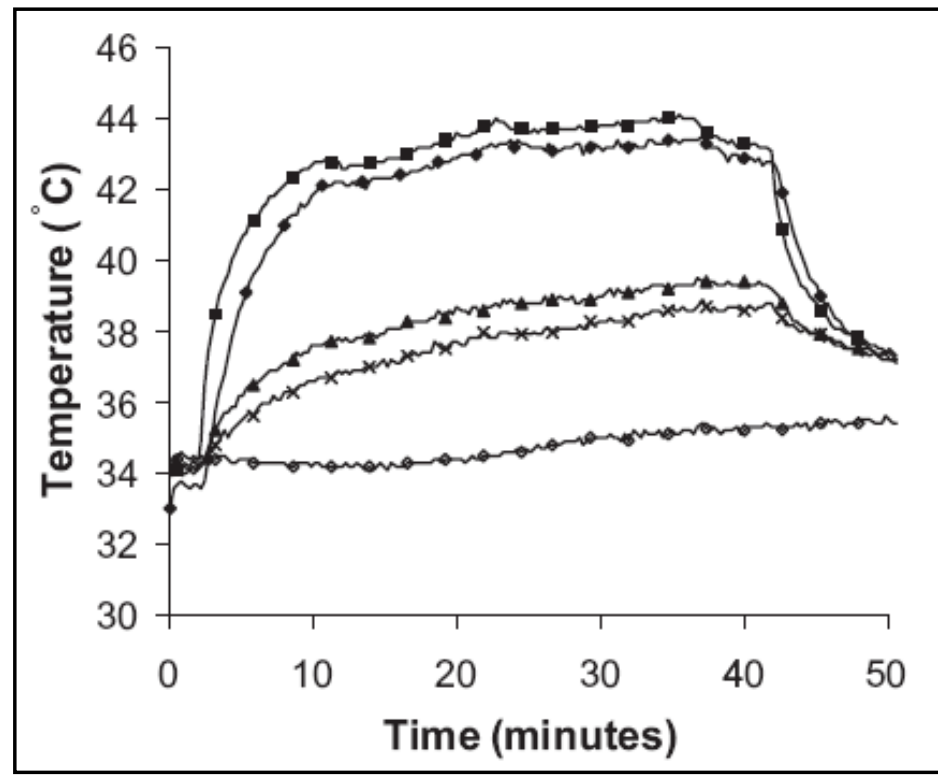

Figure 3: Temperature Results from Hyperthermia Treatment in Rabbits [3]

Studies have shown that hyperthermia treatment is effective in animals; however, little progress has been made in clinical trials because the required frequency and amplitude to create a magnetic field large enough for ideal hyperthermia conditions is beyond safe limits for humans. In 2003, a clinical feasibility study was conducted using injected superparamagnetic nanoparticles to treat glioblastoma, a form of brain cancer. Each of the fourteen patients included in this study received multiple one hour sessions with an average ferrofluid injection volume of 3 milliliters. The results of this study showed that 
maximum temperatures of 42.4 to $49.5^{\circ} \mathrm{C}$ could be achieved with only minor observable side effects. Ninety percent of the tumor reached temperatures between 39.3 to $45.5^{\circ} \mathrm{C}$ for an average of 7.7 minutes. Figure 4 shows scans of a patient's glioblastoma with injected magnetic particles [4].
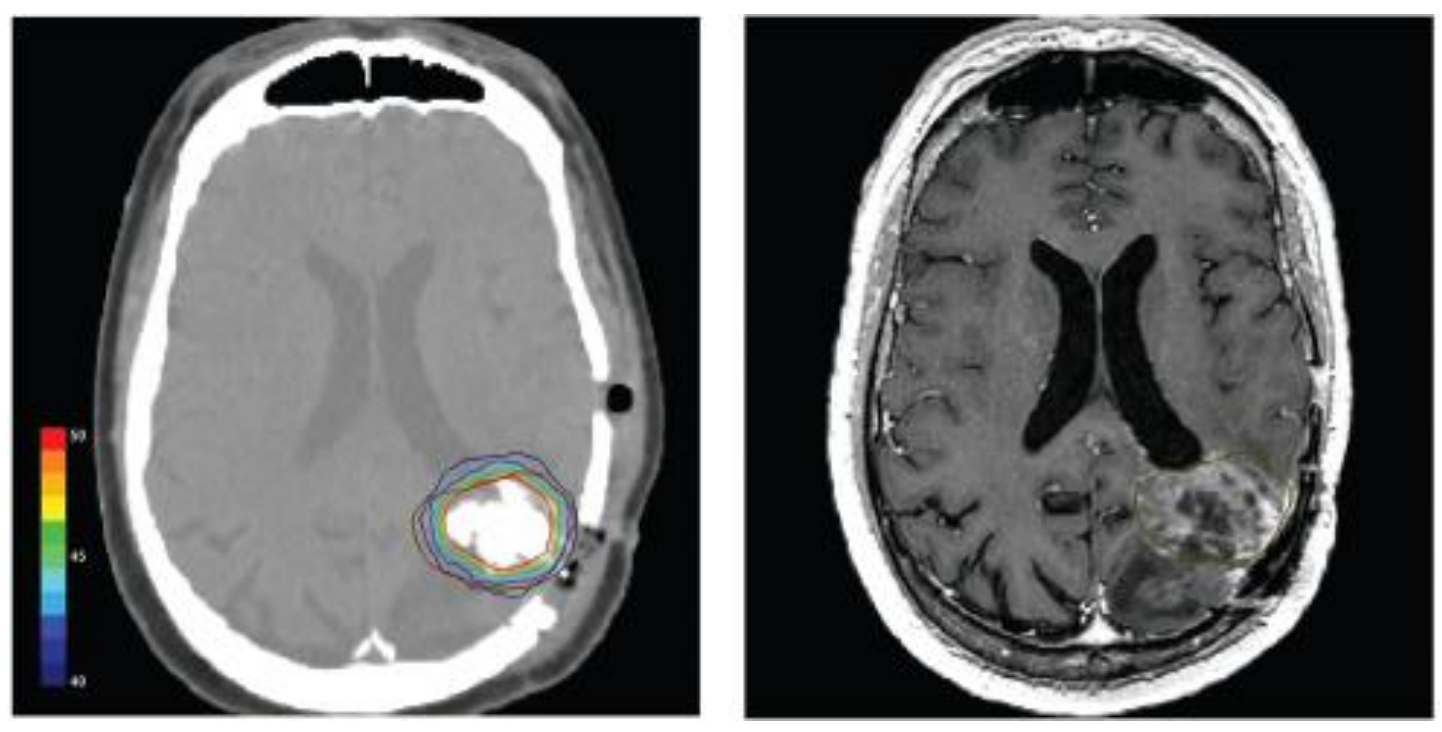

Figure 4: Scans of Glioblastoma Treatment Using Magnetic Particles [4]

Similar results were observed in two studies of magnetic particles applied to prostate cancer. In a 2004 study, magnetic particles were combined with other treatment methods for prostate cancer patients. Results indicated that although additional treatments occasionally provided slight resistance to the magnetic particles, the researchers were able to observe an average heat treatment time of 10.5 minutes. During the second prostate cancer study, researchers at the Department of Urology of the Charité at the University Medicine in Berlin were able to achieve a maximum heat treatment temperature of $55^{\circ} \mathrm{C}$ and an average dose of $43^{\circ} \mathrm{C}$ for 7.8 minutes. Following the treatment, the superparamagnetic particles were left at the treatment site. No toxicity was observed 17.5 months following injection [4]. Further testing will need to be conducted to determine the efficiency of these particles in treating cancer in humans. 


\subsection{MRI Contrasting Agents}

At the atomic level, protons will produce a small magnetic moment and align with an applied magnetic field. Since water molecules are abundant in the human body, the magnetic response of protons becomes the basis for magnetic resonance imaging (MRI). A subject in an MRI scanner will experience an alternating magnetic field through radio frequency. Water molecules within the body will absorb energy from the magnetic field and transition between energy states. When the magnetic field is turned off, the molecules will return to their original energy state, emitting energy at a frequency that is detected and converted into an image by the scanner $[1,3]$. The concentration of water molecules within various tissues results in the observed image intensities of different parts of the body.

Contrasting agents such as superparamagnetic iron oxide nanoparticles can be used to enhance MRI images by reducing the time it takes for molecules to return to their normal energy states.

With an organic coating, superparamagnetic iron oxide nanoparticles are biocompatible and travel through the body through the bloodstream. They are absorbed into the tissue by first being taken up by the reticuloendothelial system, which consists of cells lining the blood vessels that remove foreign material from the bloodstream. The reticuloendothelial system for tumor or unhealthy cells does not take up contrasting agents, thus enabling their identification. The activity of the nanoparticles in the body is dependent upon their size. Nanoparticles with diameters less than $10 \mathrm{~nm}$ are undetectable in the body and are easily taken up by the reticuloendothelial system. However, nanoparticles larger than $30 \mathrm{~nm}$ are rapidly collected by the liver and spleen and removed from the body [3]. Any nanoparticles that are not removed from the body will be naturally metabolized into iron and oxygen, both of which are used by the body.

The superparamagnetic nanoparticles used as contrasting agents are separated into two groups based on size: superparamagnetic iron oxides (SPIOs) and ultrasmall superparamagnetic iron oxides 
(USPIOs) or long-circulating dextran-coated iron oxides (LCDIOs). SPIOs have diameters greater than 50 $\mathrm{nm}$ and are currently commercially available by the product names Lumirem and Endorem. Lumirem particles, which have a diameter of $300 \mathrm{~nm}$, are used for gastro-intestinal tract imaging and function as typical contrasting agents. In comparison, Endorem particles with a diameter of $150 \mathrm{~nm}$ detect liver and spleen diseases by being taken up by healthy Kupffer cells, providing a visual contrast been healthy and diseased cells. USPIOs and LCDIOs have diameters less than $50 \mathrm{~nm}[5,6]$. Sinerem is a commercially available USPIO with a diameter of $30 \mathrm{~nm}$ and is used for blood and tumor imaging of the vascular system [5].

Superparamagnetic nanoparticles as MRI contrasting agents have been proven to be effective in both animal and clinical testing. In 2000, LCDIOs were used in rats with malignant brain neoplasms. The nanoparticles were actively taken up by tumor cells, providing clearer MRI images of the tumor. In fact, micrometastases in other locations of the body were identifiable on MRI images after taking up the nanoparticles [5]. In a clinical application of Sinerem nanoparticles to human brain tumors, the nanoparticles provided better identification of tumor cells compared to other contrasting agents such as gadolinium chelate. Due to the small size of USPIOs, these nanoparticles traverse the bloodstream longer than SPIOs. As a result, USPIOs can be used to identify lymph node diseases; however, experimental results indicate that on occasion normal and diseased tissue of the lymph node become indistinguishable as a result of rapid uptake of nanoparticles by all cells [5]. A successful application of USPIOs to a tumor of the lymph node of a rat can be seen in Figure 5 [1]. The image on the left side is prior to the injection of the USPIO contrasting agents, and the right image was taken following injection. As these images illustrate, the use of the USPIOs cause the diseased cells to become more discernable. 

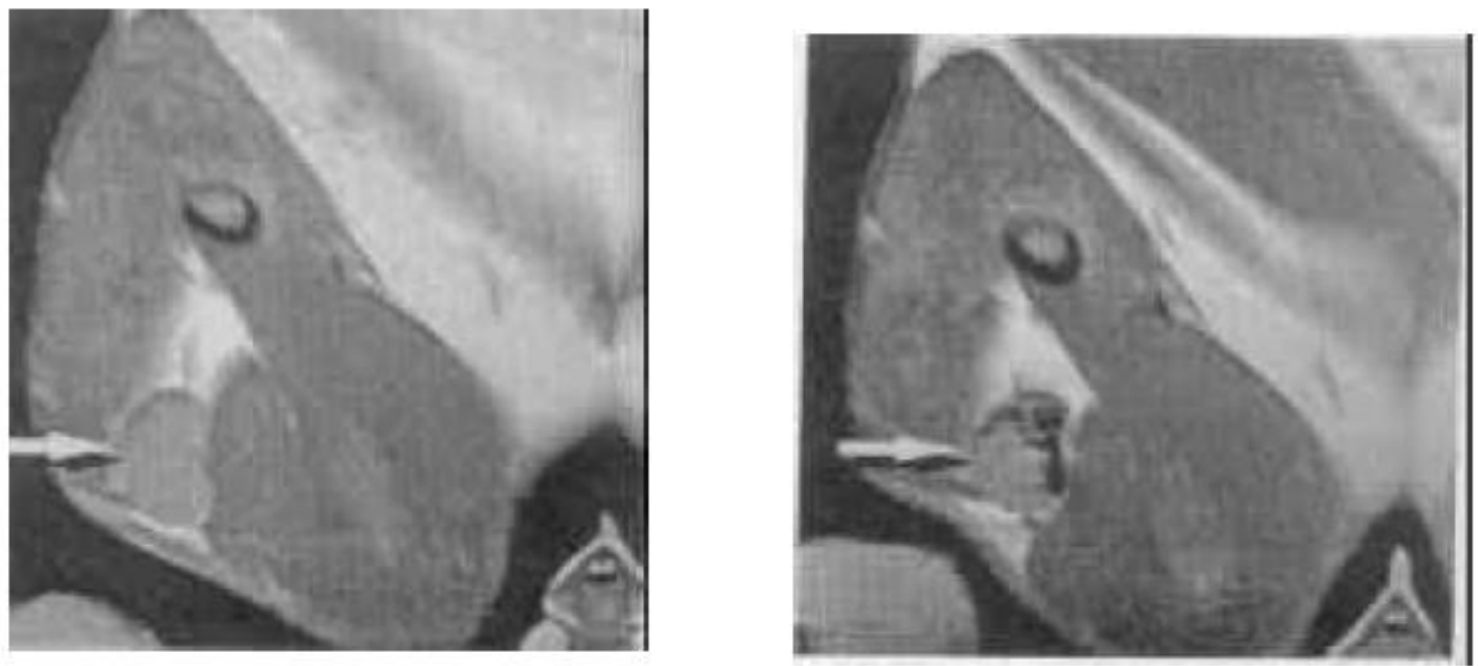

Figure 5: Superparamagnetic Particles as an MRI Contrasting Agent in a Rat Lymph Node Tumor [1]

Compared to traditional MRI contrasts such as gadolinium chelate ions, superparamagnetic nanoparticles only increase the image contrast by an order of magnitude from $10^{-5}$ moles per liter (moles/L) to $10^{-6}$ moles/L [6]. By specifically targeting diseased cells through methods such as antibodies, nucleotides, or receptor ligands, the magnitude of the contrast can be further increased, enhancing MRI images. In 2009, a study was conducted using the antibody Herceptin coated to superparamagnetic nanoparticles to target the HER2/neu receptors associated with breast cancer. In addition to successfully targeting breast cancer cells, the nanoparticles also decreased the enhancement of the cancerous cells [6].

The magnetic properties of the nanoparticles have enabled controlled release of encapsulated drugs and provided hyperthermia treatment of cancerous cells. In a 2008 study conducted by H.S. Hu et al, liposome encapsulated drugs and nanoparticles were controllably released by the application of a magnetic field while sparing any effects to healthy tissue [6]. Ji Eun Lee and colleagues experimented with the coupling of superparamagnetic nanoparticles as both an MRI contrasting agent and drug delivery method in a 2009 study by using mesoporous dye-doped silica nanoparticles decorated with 
magnetite nanocrystals and pores filled with the anti-cancer drug doxorubicin. A visual representation of these nanoparticles can be seen in Figure 6 [7].

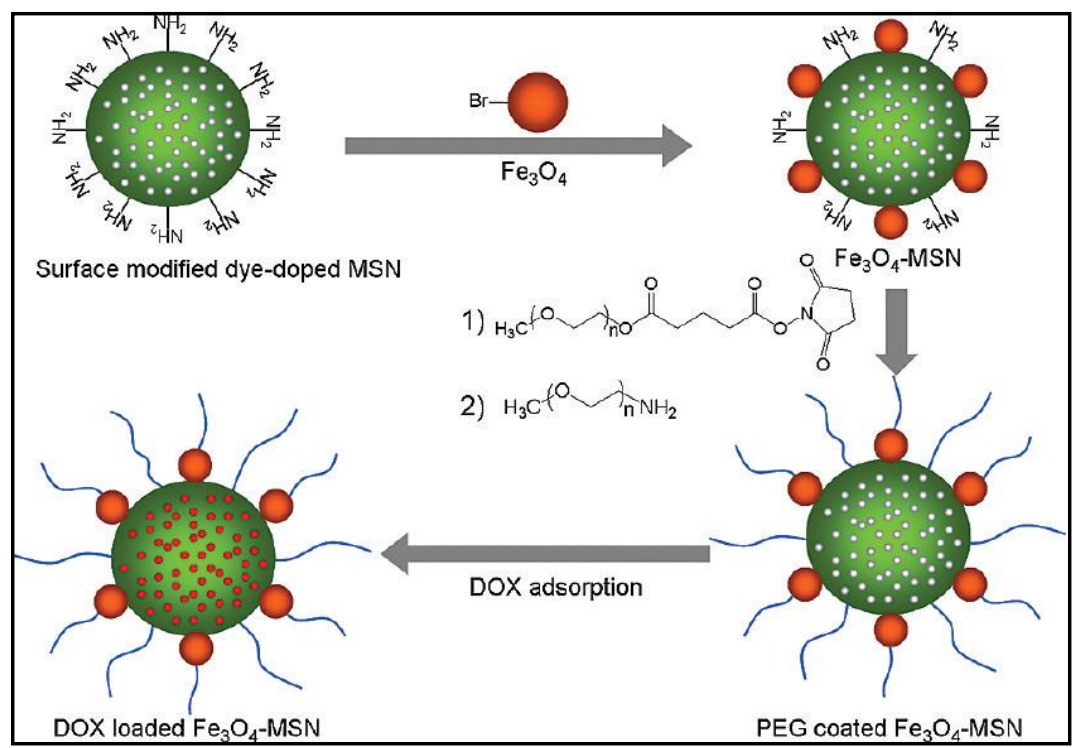

Figure 6: Representation of Functionalized Nanoparticles Used as an MRI Contrasting Agent and Drug Delivery Method [7]

The results from the study indicated that the nanoparticles were taken up via endocytosis by the tumor cells at a large concentration and were effective in being cytotoxic to the tumor cells. As Figure 7 indicates, the nanoparticles were also successful as an MRI contrasting agent, providing a method of observing diseased tissue before and after the injection [7]. 

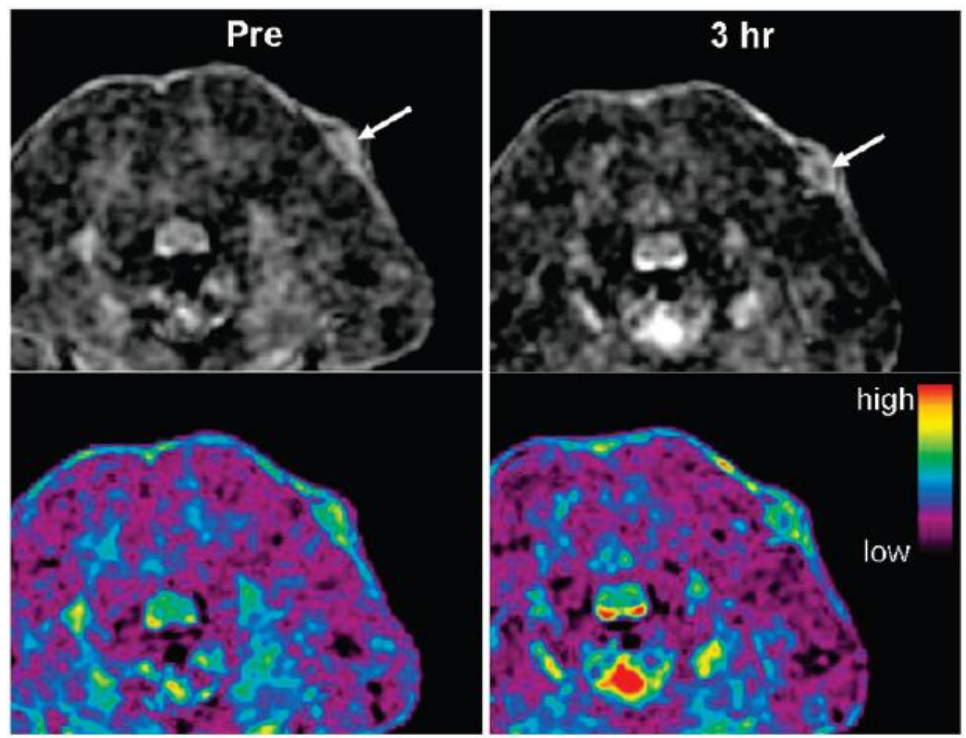

Figure 7: Scans of Cancerous Tissue Before and After the Injection of Functionalized Superparamagnetic Nanoparticles [8]

\subsection{Magnetic Separation}

The most common application of superparamagnetic particles is magnetic separation to isolate desired species. Magnetic separation is accomplished by first labeling the magnetic particles so that targeting can occur and using a fluid-based device exposed to a magnetic field gradient to separate the targeted species. Magnetic particle labeling entails coating the particle with biocompatible molecules such as dextran, polyvinyl alcohol, and phospholipids that provide a link for the binding of targeted species [3]. Once introduced into the magnetic separator, an exposed magnetic field gradient will manipulate or immobilize the magnetic particles, enabling the removal of unwanted species as fluid is continuously flowed through the separator. Figure 8 illustrates two possible magnetic separator designs [3]. The top depiction shows a separator with a magnet attached to the channel, attracting the targeted species as other species continue to flow through the channel. When the magnetic field is removed, these isolated species can then be collected. In the bottom image, steel wool is placed in the channel to aid in the capture of targeted species. 


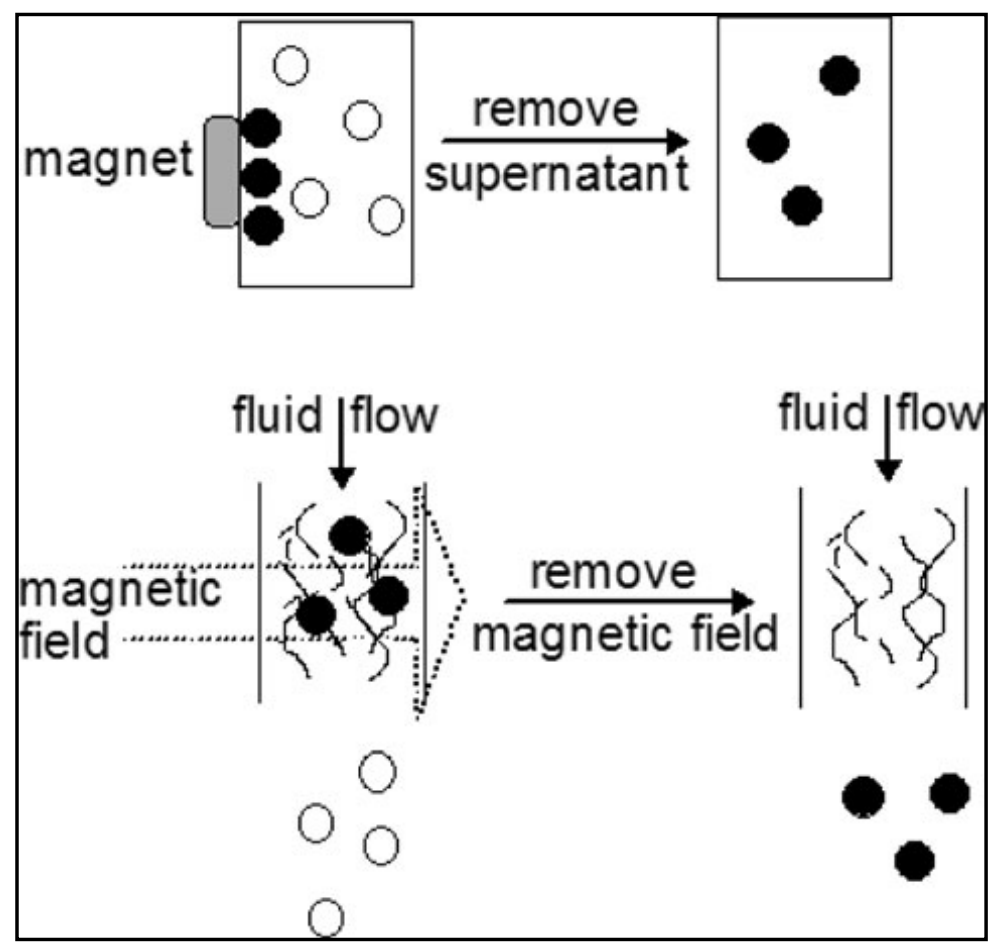

Figure 8: Possible Magnetic Separator Designs [3]

Although magnetic separation was originally applied to the isolation of magnetic color

impurities in kaolin clay and ferromagnetic impurities in boiling water, it has been successfully applied to biomolecules such as enzymes, DNA, RNA, antibodies, and magnetotactic bacteria [1]. In a 2007 study, Nicholas J. Parham et al were able to use 1.05 micron polystyrene superparamagnetic beads coated with oligonucleotides to detect 1000 genome copies of purified Group B streptococci DNA in vaginal/anal swab samples, which is responsible for sepsis and meningitis in newborns. By increasing the mer length of the oligonucleotides, they were able to improve detection. Compared to traditional screening methods, which are $87 \%$ effective for women, the use of superparamagnetic beads will provide a rapid and effective detection method that is currently being adapted for point-of-care testing [8].

A 2010 study illustrated the effective application of superparamagnetic particles to the detection and manipulation of viruses. In this study, superparamagnetic nanoparticles extracted $62 \%$ and $45 \%$ of human immunodeficiency virus type 1 virions at channel flow rates of 10 and $30 \mu \mathrm{L} / \mathrm{min}$, 
respectively. As seen in Figure 9, the nanoparticles and virion samples are introduced through the two inlets located on one end of the microfluidic device before mixing in a passive mixer to provide binding of functionalized nanoparticles with the viral proteins. From the micromixer, the sample reaches the magnetic separator which consists of randomly placed iron particles trapped in the microfluidic chamber and a neodymium (NdFeB) magnet outside the device. The iron particles serve as magnetic field gradient concentrators that produce areas of high magnetic field gradients due to irregular shapes and angles, thus providing areas for bound nanoparticles to become trapped by the embedded iron particles [9].

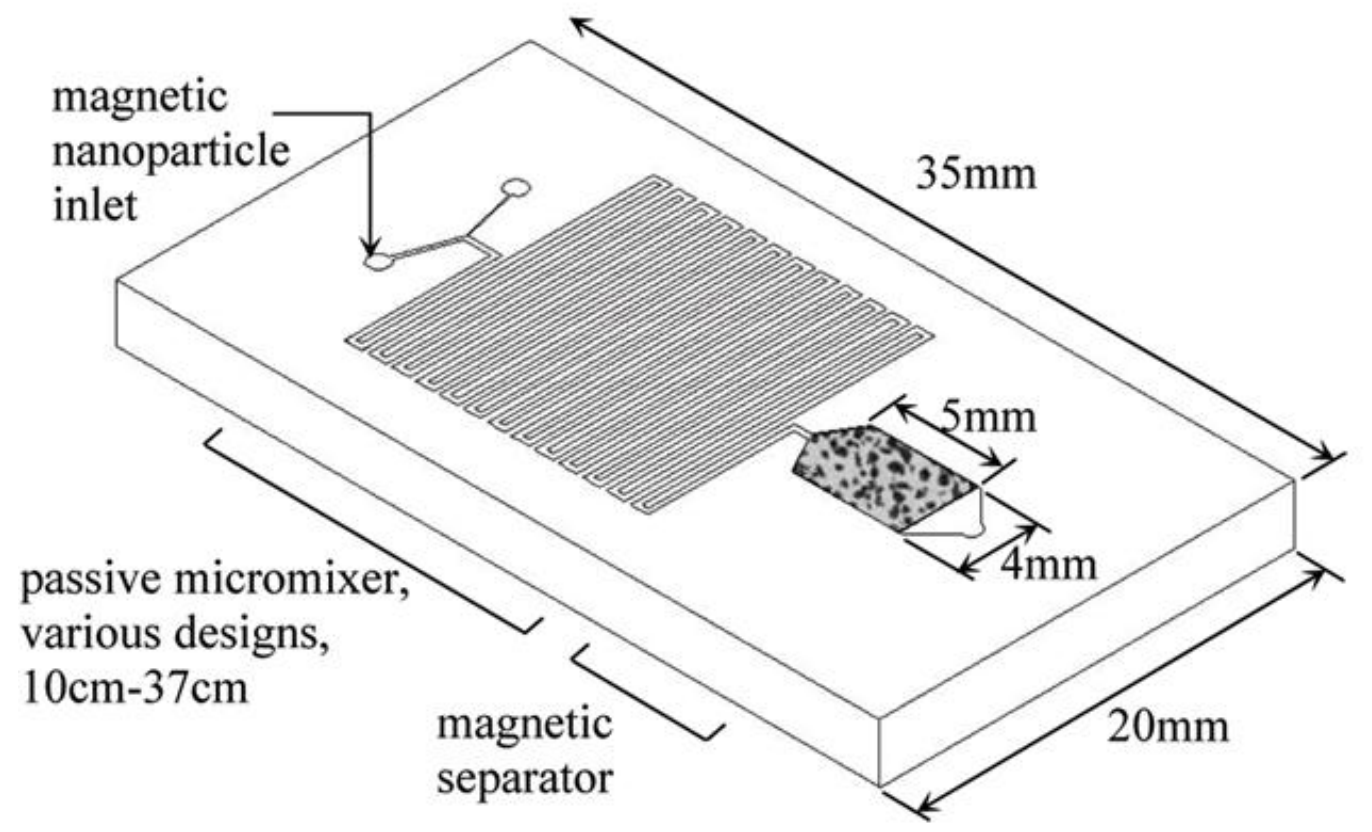

Figure 9: Micromixer and Magnetic Separator Used for HIV Type 1 Virion Detection [9]

\subsection{Modeling of Superparamagnetic Particles}

To aid with the study and application of superparamagnetic particles to the above applications, computer modeling of the particles using the finite element analysis and multiphysics simulation software $\mathrm{COMSOL}^{\circledR}$ has become a common practice. $\mathrm{COMSOL}^{\circledR}$ provides an excellent starting point towards understanding the movement of superparamagnetic particles under various controllable settings before moving towards experimental studies. 
Since targeted drug delivery is such a promising area of research, $\mathrm{COMSOL}^{\circledR}$ has created a twodimensional library model illustrating the physics of ferrofluids in the presence of a magnetic field. In this model, which is illustrated in Figure 10, the ferrofluid flows through a blood vessel located between two layers of human tissue at a velocity corresponding to the pumping of blood through the vessel. A permanent magnet rests upon the top layer of human tissue [10].

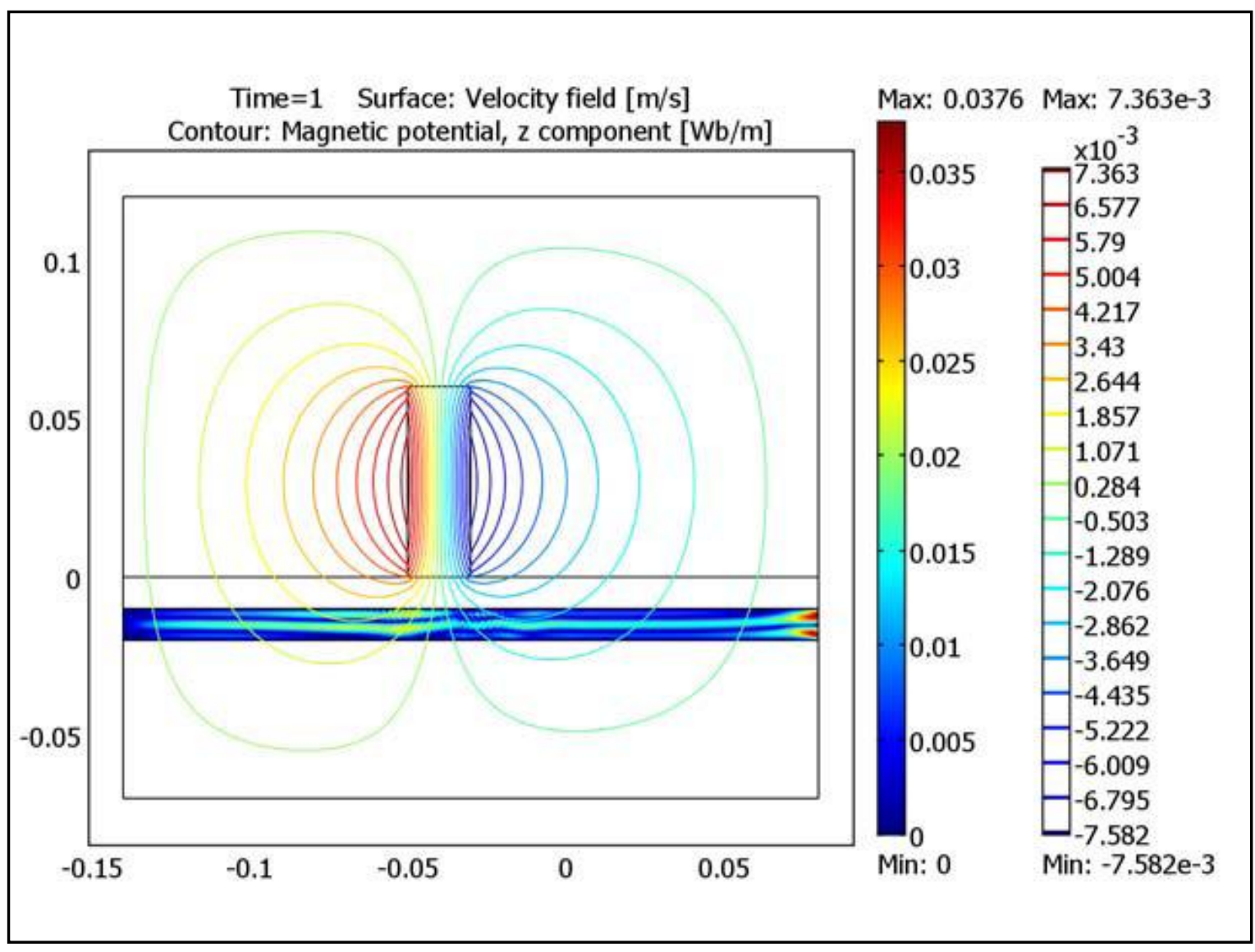

Figure 10: $\mathrm{COMSOL}^{\circledR}$ Model of Ferrofluid in Bloodstream [10]

The above model is accomplished by coupling the Incompressible Navier-Stokes multiphysics mode with the Magnetostatics mode. Internally, $\mathrm{COMSOL}^{\circledR}$ computes the magnetic field of the model based on the parameters of the permanent magnet, and this magnetic field affects the fluid flow in the vessel through a resultant magnetic volume force [10]. 
The Department of Chemistry at West Virginia University has also developed a model of superparamagnetic particles. In this simulation, the group sought to model the movement of individual particles instead of a ferrofluid by coupling the Magnetostatics, Incompressible Navier-Stokes, and Moving Mesh Arbitrary Lagrange-Eulerian (ALE) modes. As with the $\mathrm{COMSOL}^{\circledR}$ ferrofluid model, the Magnetostatics mode calculated the magnetic field produced by the permanent magnet. However, the Incompressible Navier-Stokes mode was also necessary to provide the Moving Mesh ALE mode with information necessary to track the movement of the superparamagnetic particles towards the permanent magnet. Unlike the model of the ferrofluid, the particles in this model did not endure a velocity field as a result of moving fluid; instead, the Incompressible Navier-Stokes mode calculated the velocity of the particles as a result of their movement in response to the volumetric magnetic force and the opposing viscous drag force exerted on them by the magnetic field and surrounding fluidic environment, respectively. An illustration of this model can be seen in Figure 11 [11]. 


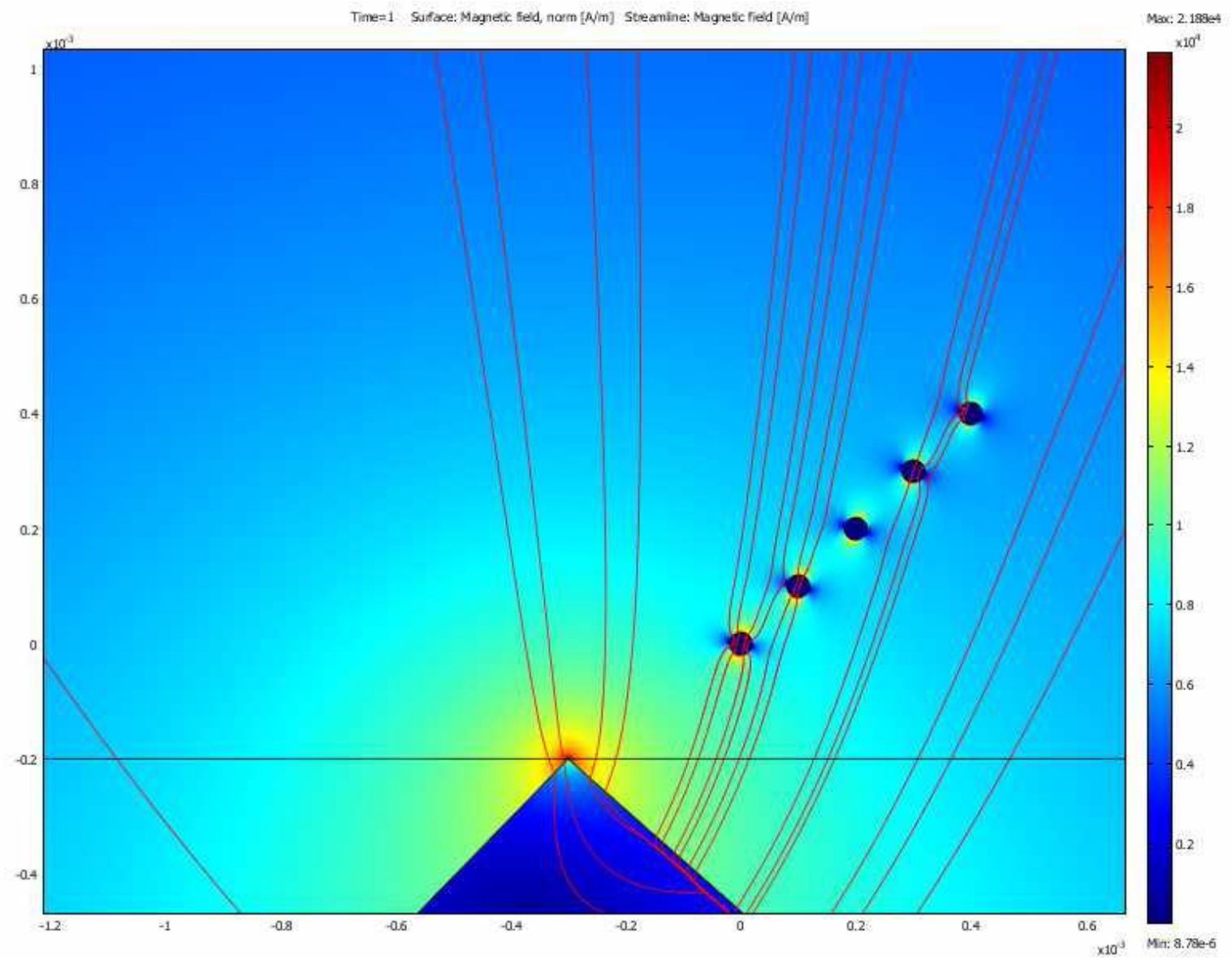

Figure 11: Previous $\mathrm{COMSOL}^{\circledR}$ Modeling of Individual Superparamagnetic Beads [11]

As the above image illustrates, the West Virginia University group was successful in depicting the movement of individual beads in the presence of a magnetic field. In Figure 11, the bright coloring at the apex of the permanent magnet represents the location of the highest magnetic field. Corresponding coloring on the five superparamagnetic beads identifies the resultant magnetization that each bead exhibits in response to the presence of other superparamagnetic beads. The semi-vertical streamlines represent the magnetic field produced by the permanent magnet with the streamlines aligning with the beads and highlighting their path towards the magnet.

For the detection of toxic particles in the bloodstream, Michael Mayer et al developed a magnetic trapping system and created an associated $\mathrm{COMSOL}^{\circledR}$ model. Their system consists of fluorescently labeled superparamagnetic particles immersed with toxic particles within a filter channel 
for the purification of blood. If the filter breaks and releases toxic particles into the bloodstream, the presence of the superparamagnetic particles enables the detection of the toxic particles through optical imaging. A magnetic trapping system consisting of two magnets, as seen in Figure 12, enables the isolation of the superparamagnetic particles for optical detection [12].

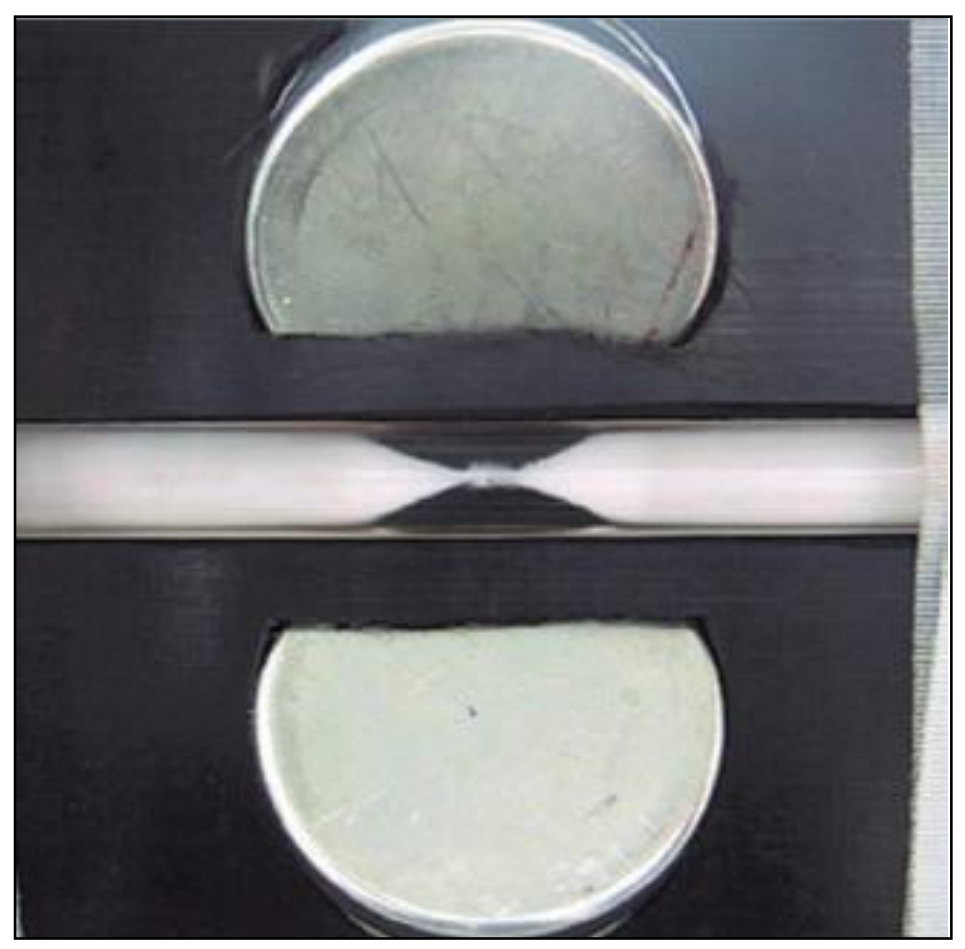

Figure 12: The Magnetic Trapping System Used for Toxic Particle Detection [12]

Similar to the model of the ferrofluid, the $\mathrm{COMSOL}^{\circledR}$ model for the magnetic trapping system uses the coupling of the Incompressible Navier-Stokes and Magnetostatics modes to simulate the trapping of the superparamagnetic particles. Unlike previous models, lift of the particles is included in the resultant forces of the system. As can be seen by Figure 13, the model showed results similar to those observed experimentally in Figure 12 with the magnetic particles accumulating in the channel between the permanent magnets [12]. 


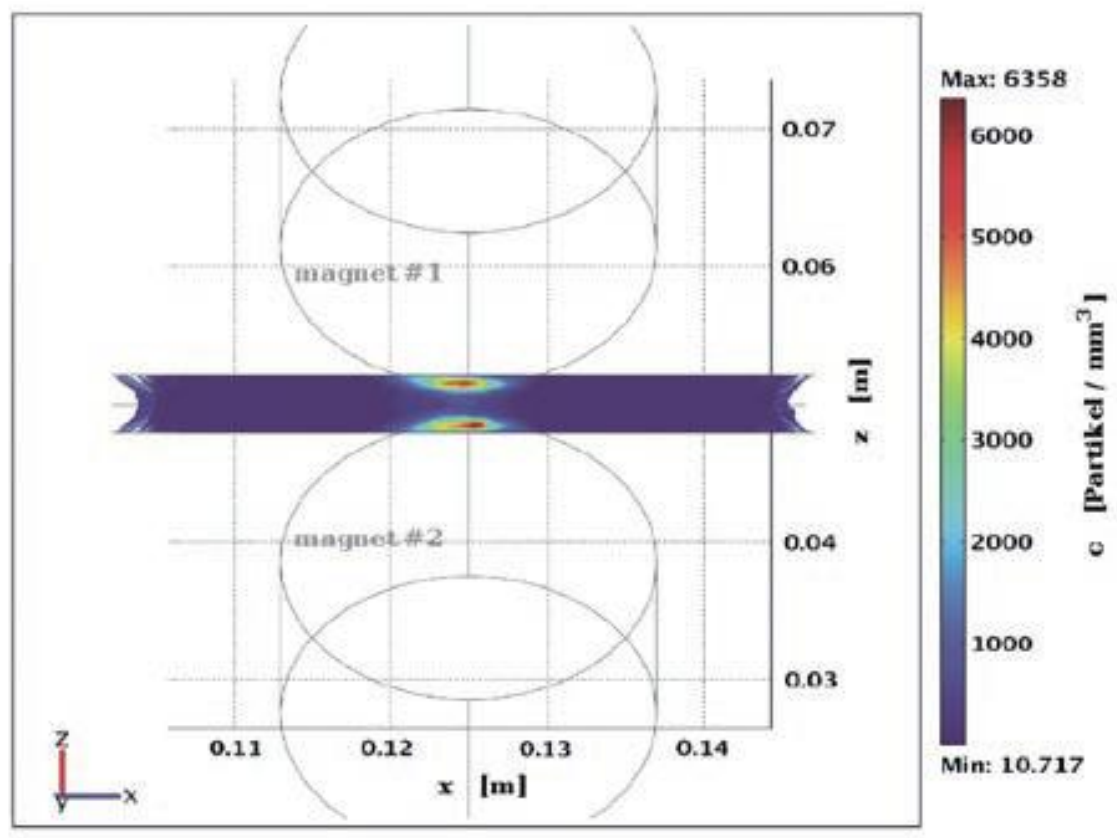

Figure 13: $\mathrm{COMSOL}^{\circledR}$ Model of Magnetic Trapping System Used for Toxic Particle Detection [12]

For magnetic trapping and separation, the coupling of the Incompressible Navier-Stokes and Magnetostatics modes provides the foundation for the modeling of magnetic separators by illustrating the movement of a ferrofluid through a channel, and the incorporation of the Moving Mesh ALE mode enables individual identification and observation of superparamagnetic beads. However, a $\mathrm{COMSOL}^{\circledR}$ model that allows the quantifiable efficiency of a magnetic separator has yet to be introduced. In the following sections, the creation of such a model will be developed and introduced by first identifying the design for the magnetic separator and the physics behind the model.

\subsection{The Magnetic Separator}

A simple magnetic trapping system designed for diagnostics is used as the basis for the $\mathrm{COMSOL}^{\circledR}$ models. This system, which can be seen graphically in Figure 14 , consists of a microfluidic channel beneath a stationary permanent magnet. Functionalized superparamagnetic beads are introduced into the microfluidic channel through the inlet and are attracted towards the permanent magnet at the top of the channel. As the beads are trapped by the magnetic field, fluorescent tracer 
reagents are introduced to bind to the beads. The bound beads are then released from the system by removing the permanent magnet and analyzed using optical detection to determine the capture and release efficiencies of the system.

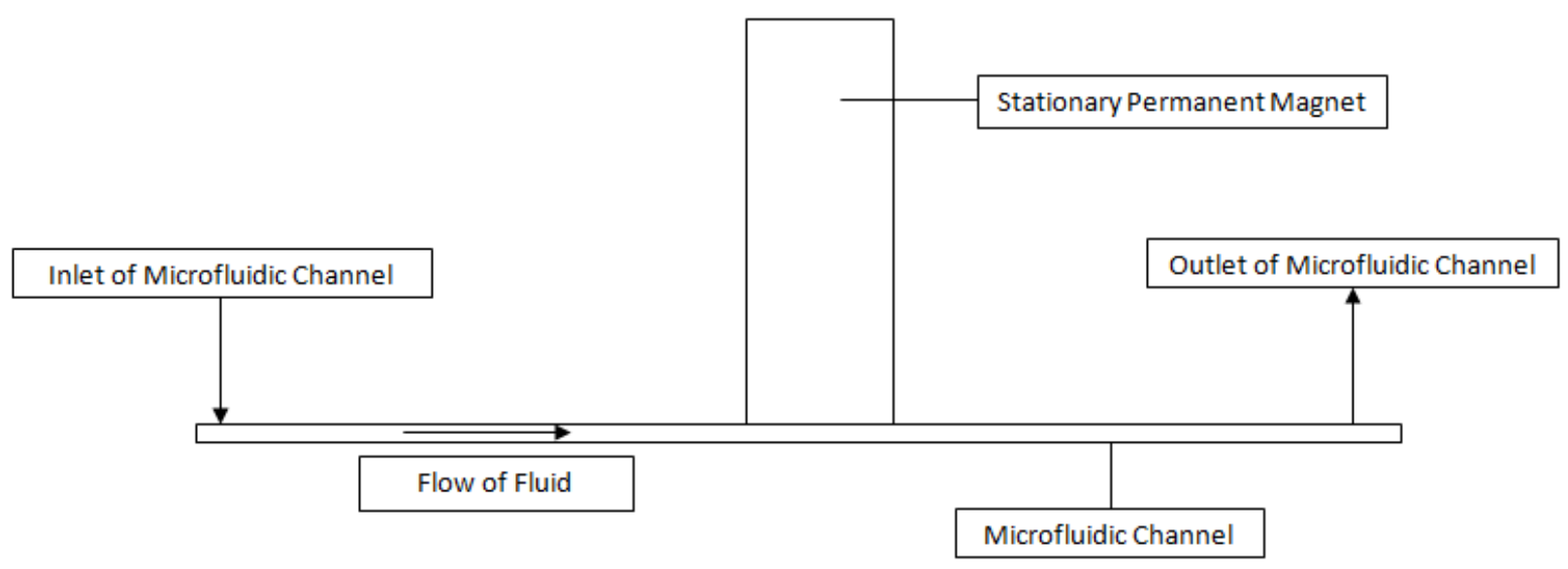

Figure 14: Graphic Depiction of the Magnetic Trapping System

Experimentally, determining the capture and release efficiencies of the system would be a timeconsuming process, involving the manipulation of several parameters. In an attempt to understand the system prior to experimentation, $\mathrm{COMSOL}^{\circledR}$ modeling of this system was conducted based on the ferrofluid library model with the coupling of the Incompressible Navier-Stokes and Magnetostatics modes. In this model, a thin channel is placed beneath a stationary magnet with parameters representative of the experimental system. The results from this model can be seen in Figure 15 where the magnetic potential of the magnet and velocity field of the fluid are depicted. 


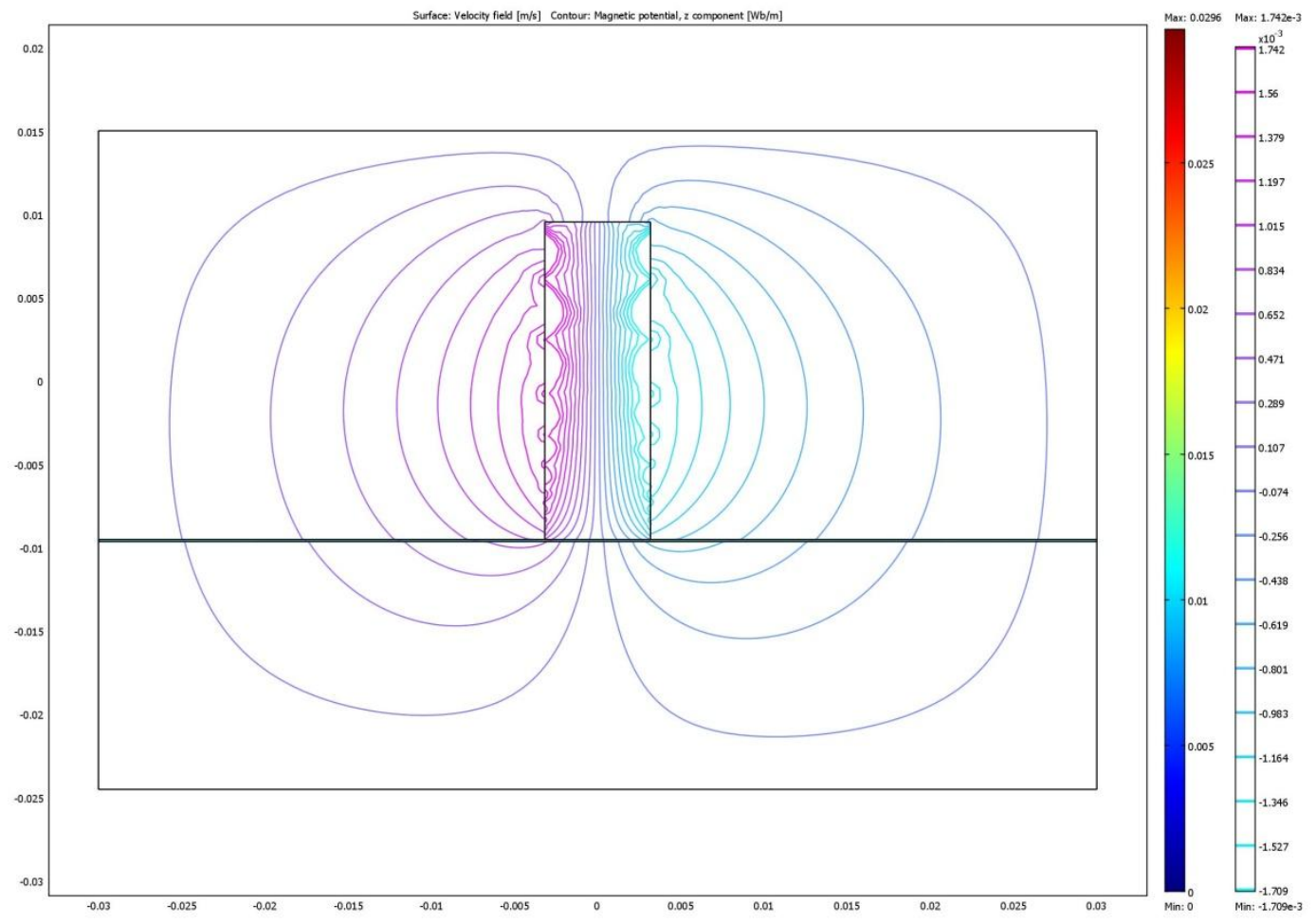

Figure 15: $\mathrm{COMSOL}^{\circledR}$ Modeling of the Magnetic Separator

Such a model is useful in illustrating the magnetic potential and velocity of the system but did not provide much information on the movement of the beads in the system aside from disturbances in the velocity field. A closer look of the microfluidic channel beneath the permanent magnet can be seen in Figure 16. 


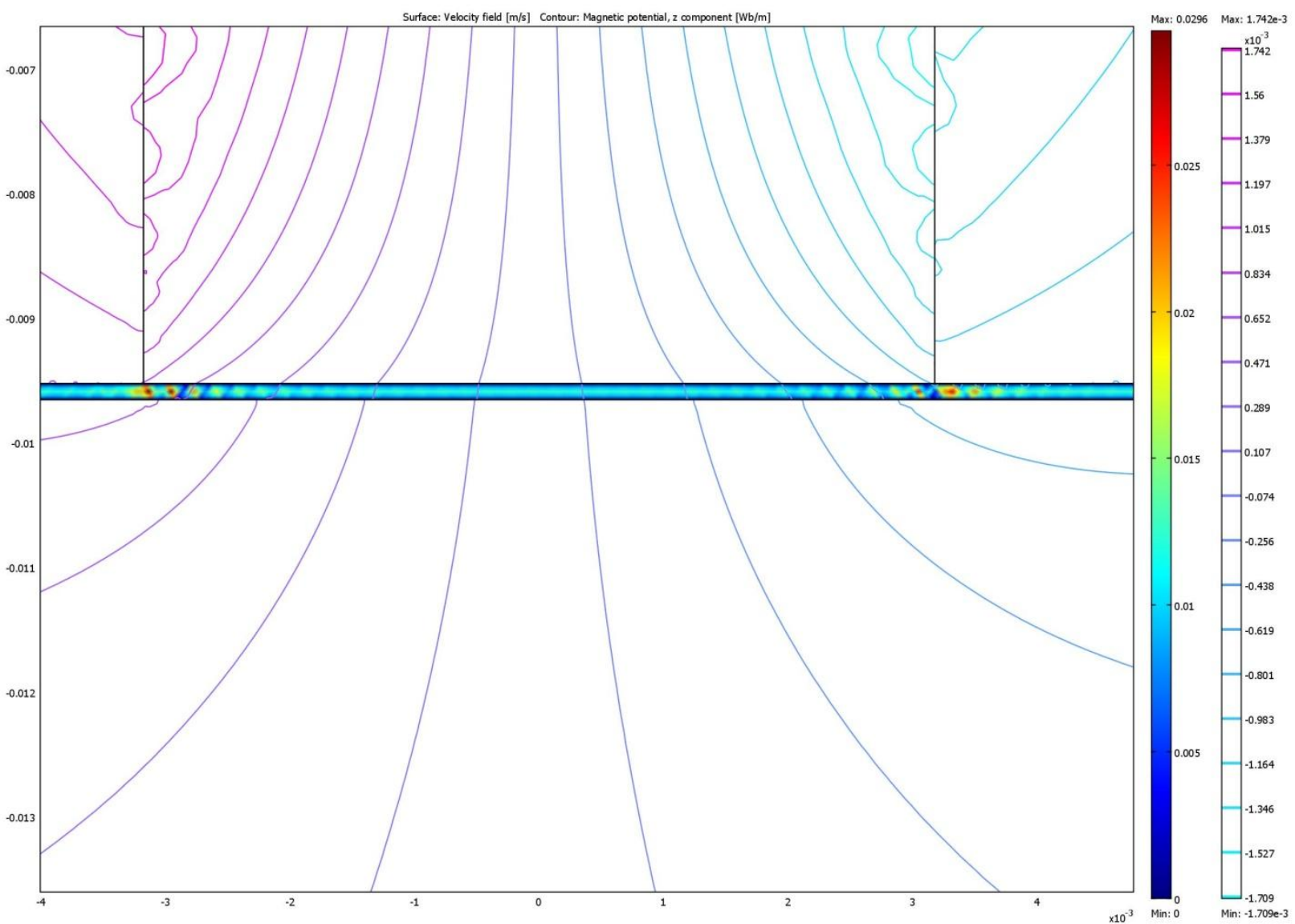

Figure 16: A Larger View of the Microfluidic Channel Beneath the Stationary Magnet

This system and the $\mathrm{COMSOL}^{\circledR}$ model already created for it will be built upon in the following sections with the introduction of a new $\mathrm{COMSOL}^{\circledR}$ model for monitoring superparamagnetic bead movement in a magnetic separator.

\subsection{The Objectives}

The objectives of the following chapters are to develop a $\mathrm{COMSOL}^{\circledR}$ model that enables: 1) a depiction of the movement of superparamagnetic particles in a microfluidic channel exposed to a magnetic field, 2) the quantification of the particles in the microfluidic channel at any location and time, and 3) a method for comparing different model settings for design optimization. To measure these objectives, visual inspection and the use of Post-Processing tools will be used. Visually, a model will be 
deemed successful in regard to the first objective if a concentration or number density of superparamagnetic particles can be traced and followed through the microfluidic channel over a period of time. The second and third objectives will be accomplished if a quantifiable amount of the superparamagnetic particles can be determined at any location and time in the microfluidic channel through Post-Processing plots. By changing the parameters of the model, the Post-Processing results should provide a comparison for design optimization. 


\section{Chapter 2: Fluid Flow Theory}

\subsection{Microfluidics}

Microfluidics is a research field that involves the manipulation of small amounts of fluids within device channels with dimensions on the micrometer to millimeter scale. Although this field dates back to capillary experiments performed by Hagen and Poiseuille, extensive knowledge and use of this field did not begin until the emergence of microelectromechanical systems (MEMS) in the early 1980's, which provided the foundation for microfluidics. MEMS sought to incorporate both electrical and mechanical elements such as sensors and actuators on a single substrate for portable and integrative devices. MEMS that focus exclusively on the use of fluid as the primary medium are microfluidic devices [13].

This field of research offers many advantages compared to large-scale systems. The small size of the system reduces the amount of reagents used, has inexpensive fabrication techniques, reduces analysis times, and enables the complex integration of multiple steps to create portable lab-on-a-chip systems. In addition, the small size and integration of other components such as electronics enable predictable manipulation of the fluid. Blood samples, bacterial cell suspensions, protein and antibody solutions, and various buffers are common fluids used in microfluidic devices. These devices are used in a wide range of applications; some common applications include: inkjet heads, protein crystallization, proteonomics, micropumps, sample pretreatment, molecular diagnostics, capillary electrophoresis, isoelectric focusing, immunoassays, flow cytometry, PCR amplification, DNA analysis, cell manipulation and separation, and clinical diagnostics [14-16].

\subsection{Fluid Theory}

A fluid is any substance that continuously deforms in response to a tangential, or shearing, force applied to its surface and encompasses gas, liquid, and plasma states of matter. It is commonly treated as a continuum, or averaged property, since the molecular composition of fluids results in numerous 
individual behaviors. Fluids are characterized by several properties including: density, specific weight, pressure, viscosity, and surface tension. A summary of these properties will be discussed in this section. Any standard fluid textbooks such as references $[17,18]$ can provide further information.

The density, which has units of kilograms per cubic meter $\left(\mathrm{kg} / \mathrm{m}^{3}\right)$, represents the mass per volume of the fluid. For liquids, the density does not vary significantly depending on pressure; however, the density of gases changes drastically with pressure. A fluid that has a constant density is known as an incompressible fluid. Compressible fluids have densities that vary with changes in temperature and pressure.

Similar to density, the specific weight is the weight per unit volume of a fluid with units of newton per cubic meter $\left(\mathrm{N} / \mathrm{m}^{3}\right)$. It is the product of a fluid's density and gravity. Since the density of liquids is greater than that of gases and vapors, the specific gravity of liquids is also larger.

Pressure is the normal force per area of a fluid at rest and has units of newton per meter squared $\left(\mathrm{N} / \mathrm{m}^{2}\right)$. The pressure does not vary horizontally within a fluid, but it does vary vertically depending upon the type of fluid. The pressure of an incompressible fluid varies linearly with depth since its density does not vary with location or pressure. In comparison, the pressure of compressible fluids remains relatively constant over small changes in vertical depth of the fluid because the specific weight of compressible fluids is very small compared to incompressible fluids. With large vertical distances, this pressure becomes non-linear since the lower fluid is more dense than fluid located higher vertically.

The viscosity of a fluid is a measure of its resistance to continuous deformation. There are two types of viscosities: dynamic and kinematic; in future sections, discussions of viscosities will be referring to dynamic viscosity. A fluid located within a channel such as a microfluidic device experiences a velocity gradient as a result of deformation that can come in the form of the fluid's velocity or the shearing stress of a moving wall of the channel. For stationary walls, the fluid tends to stick to the boundary of 
the channel, resulting in what is referred to as a no slip condition. If both walls of the channel are stationary, this produces a parabolic velocity profile. For channels with one wall moving, the velocity gradient is linear, producing a Couette flow. The shearing stress $\tau$ is related to the velocity gradient through the following relationship:

$$
\tau=\mu \frac{d u}{d y}
$$

where $\frac{d u}{d y}$ is the velocity gradient and $\mu$ is the dynamic viscosity of the fluid with units of newton second per meter squared $\left(\mathrm{Ns} / \mathrm{m}^{2}\right)$. Fluids can be classified based on the relationship between the shearing stress and velocity gradient. Newtonian fluids are fluids with a linear relationship between the shearing stress and velocity gradient. Conversely, the relationship is non-linear for non-Newtonian fluids. Water and air are common examples of Newtonian fluids, and blood is a common example of a non-Newtonian fluid used in microfluidics devices. The kinematic viscosity $v$ of a fluid is determined by:

$$
v=\frac{\mu}{\rho}
$$

where $\rho$ is the density of the fluid. The kinematic viscosity has units of meters per second squared $\left(\mathrm{m} / \mathrm{s}^{2}\right)$

The individual molecules of a liquid are attracted to each other. Within the interior of the liquid, these attractive forces are equally balanced, resulting in no net attractive forces. On the surface of the liquid, however, these cohesive forces between liquid molecules are unequal, producing a net attraction towards the interior of the liquid. This attraction is known as surface tension with units of newton per meter $(\mathrm{N} / \mathrm{m})$. The surface tension for a given liquid depends on the fluid it makes contact with and also varies inversely with temperature.

With microfluidics, the fundamental physics of fluids decrease rapidly from those observed macroscopically. At this small scale, the surface area of a fluid is much larger than its volume, making mass and heat transfer more efficient and the surface tension of the fluid much more influential on the 
fluid's properties. As a result, the mass transport within the fluid is dominated by viscous effects, while the inertial effects are negligible in comparison.

\subsubsection{Reynolds Number}

The Reynolds number, named after the British engineer Osborne Reynolds, is a dimensionless number that relates the effects of inertial forces to those of viscous forces on a fluid. It can be used to determine the flow regime of the fluid and is calculated by:

$$
R e=\frac{\rho u l}{\mu}
$$

where $u$ is the velocity and $l$ is the characteristic length of the microfluidic channel. For circular channels, $l$ is the diameter of the channel; for non-circular channels, $l$ is:

$$
l=\frac{4 A}{P}
$$

where $A$ is the cross section and $P$ is the wetted perimeter of the channel. There are four types of flow regimes: laminar, turbulent, transition, and transient Stokes flow. With laminar flow, the layers of fluid flow in parallel to one another. Turbulent flow possesses cross currents that result in eddies and swirls of the fluid. Transitional flow is characterized by the presence of both laminar and turbulent fluid flow. Transient Stokes flow is characterized by the equation that will be discussed in the next section.

As the equation indicates, a large Reynolds number indicates that the inertial forces dominate the viscous forces. If the Reynolds number becomes large enough, the fluid becomes unstable as turbulent flow results. For microfluidic devices, the dimensions and velocity of the channels are such that the Reynolds number is very small. At small numbers, the viscous forces dominate the inertial forces to the point where inertial forces become negligible. As a result, a laminar flow regime can be observed within the microchannel. Table 1 illustrates the fluid types and flow regimes associated with various ranges of Reynolds numbers [19]. 
Table 1: Reynolds Numbers of Various Fluids and Flow Regimes [19]

\begin{tabular}{|c|c|c|}
\hline Fluid Type & Flow Regime & Reynolds Number \\
\hline Compressible Fluids (Gases) & Laminar Flow & $\operatorname{Re}<100$ \\
\hline Incompressible Fluids (Liquids) & Transient Stokes Flow & $\operatorname{Re}<<1$ \\
\hline Incompressible Fluids & Laminar Flow & $\operatorname{Re}<1000$ \\
\hline Incompressible Fluids & Transition Flow & $2000<\operatorname{Re}<4000$ \\
\hline Incompressible Fluids & Turbulent Flow & $\operatorname{Re}>4000$ \\
\hline
\end{tabular}

For most microfluidics devices, the Reynolds number is below 1, resulting in a transient Stokes flow and laminar regime. Also, the inertial forces can be neglected for the system.

\subsubsection{Navier-Stokes Equation}

For Newtonian fluids, the velocity field can be described using the Navier-Stokes equations, which are derived from Newton's Second Law:

$$
\rho\left(\frac{\partial \boldsymbol{u}}{\partial t}+\boldsymbol{u} \cdot \nabla \boldsymbol{u}\right)=-\nabla p+\boldsymbol{F}+\mu\left(\nabla^{2} \boldsymbol{u}\right)
$$

where $\boldsymbol{F}$ is a volumetric body force exerted on the fluid. Equation 2.5 can be expanded to the following:

$$
\begin{gathered}
\rho\left(\frac{\partial u}{\partial t}+u \frac{\partial u}{\partial x}+v \frac{\partial u}{\partial y}+w \frac{\partial u}{\partial z}\right)=-\frac{\partial p}{\partial x}+F_{x}+\mu\left(\frac{\partial^{2} u}{\partial x^{2}}+\frac{\partial^{2} u}{\partial y^{2}}+\frac{\partial^{2} u}{\partial z^{2}}\right) \\
\rho\left(\frac{\partial v}{\partial t}+u \frac{\partial v}{\partial x}+v \frac{\partial v}{\partial y}+w \frac{\partial v}{\partial z}\right)=-\frac{\partial p}{\partial y}+F_{y}+\mu\left(\frac{\partial^{2} v}{\partial x^{2}}+\frac{\partial^{2} v}{\partial y^{2}}+\frac{\partial^{2} v}{\partial z^{2}}\right) \\
\rho\left(\frac{\partial w}{\partial t}+u \frac{\partial w}{\partial x}+v \frac{\partial w}{\partial y}+w \frac{\partial w}{\partial z}\right)=-\frac{\partial p}{\partial z}+F_{z}+\mu\left(\frac{\partial^{2} w}{\partial x^{2}}+\frac{\partial^{2} w}{\partial y^{2}}+\frac{\partial^{2} w}{\partial z^{2}}\right)
\end{gathered}
$$

where $u, v$, and $w$ are the $\mathrm{x}, \mathrm{y}$, and $\mathrm{z}$ components of the fluid's velocity. Since the inertial forces can be neglected, Equation 2.5 becomes:

$$
\rho\left(\frac{\partial \boldsymbol{u}}{\partial t}\right)=-\nabla p+\boldsymbol{F}+\mu\left(\nabla^{2} \boldsymbol{u}\right)
$$

Four possible forces act on the superparamagnetic beads within a microchannel: the weight of the bead $\mathbf{F}_{\mathrm{g}}$, buoyancy force $\mathbf{F}_{\mathrm{b}}$, drag $\mathbf{F}_{\mathrm{d}}$, and magnetic force $\mathbf{F}_{\mathrm{m}}$. These forces are depicted in Figure 17 and will be further discussed below. 


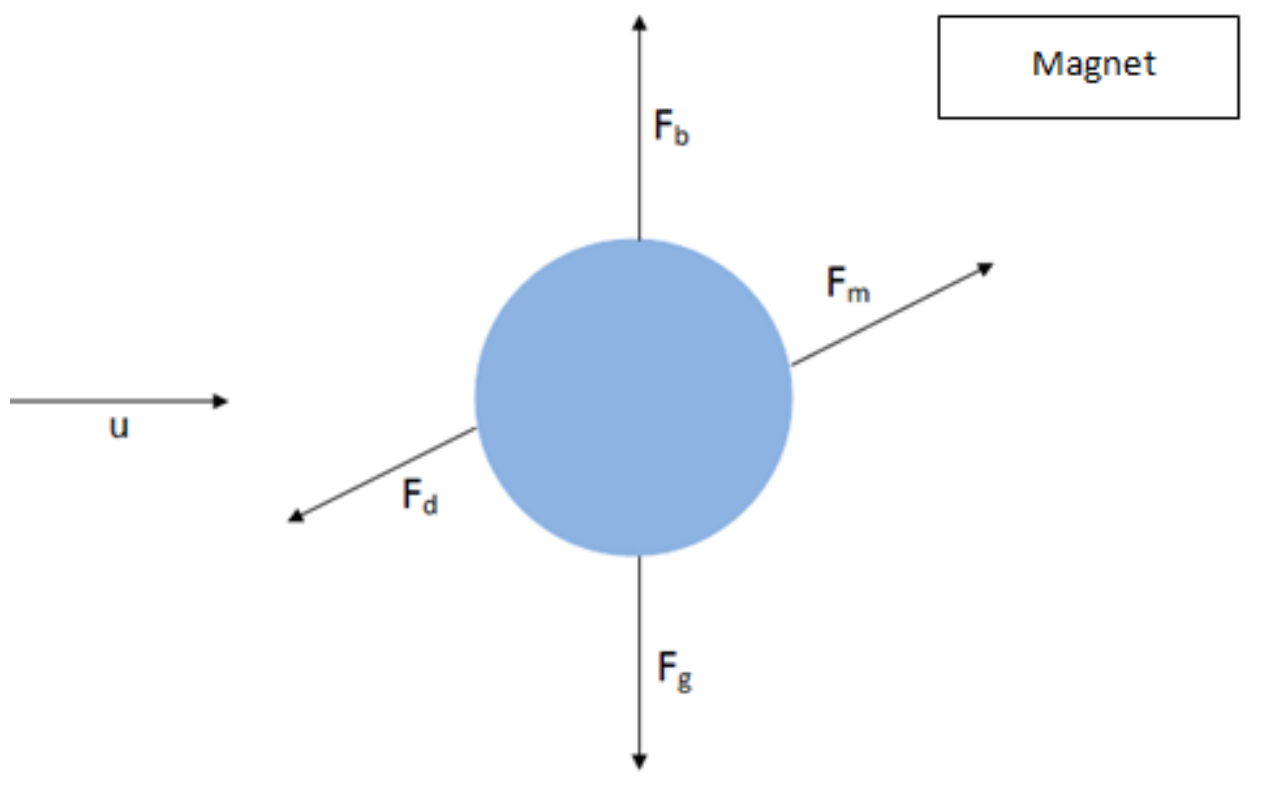

Figure 17: The Forces Exerted on a Superparamagnetic Bead in a Microfluidic Channel

\subsubsection{Weight of the Bead}

The weight of the bead $\mathbf{F}_{\mathrm{g}}$ is determined through the following equation:

$$
\boldsymbol{F}_{g}=m g
$$

where $m$ is the mass of the bead and $g$ is the acceleration of gravity. However, since inertial effects are neglected for microfluidic systems, the weight of the bead is also neglected.

\subsubsection{Archimedes' Principle}

For macroscopic circumstances, an object submerged in a fluid would experience a floating or buoyancy force from the surrounding fluid. According to Archimedes' Principle, this buoyancy force is equal to the weight of the fluid and is directed vertically upward on the body of the object by the fluid. This principle can be expressed as:

$$
\boldsymbol{F}_{B}=\gamma V
$$


where $\gamma$ is a the specific gravity and $V$ is the volume of the object. For the superparamagnetic beads included in the microfludic system discussed in this paper, inertial effects can be neglected, indicating that the buoyancy force of the beads can also be neglected.

\section{$\underline{2.2 .2 .3 \text { Viscous Drag }}$}

Stoke's Law describes the hydrodynamic forces acting on moving spherical particles within a fluid with a small velocity and a Reynolds number much less than one. For a superparamagnetic bead, which is spherical in shape, its viscous drag is determined by:

$$
\boldsymbol{F}_{d}=-6 \pi \mu a \Delta v
$$

where $a$ is the diameter and $\Delta \boldsymbol{v}$ is:

$$
\Delta \boldsymbol{v}=\boldsymbol{v}_{\text {Particle }}-\boldsymbol{v}_{\text {Fluid }}
$$

where $\boldsymbol{v}_{\text {Fluid }}$ is the velocity of the surrounding fluid and $\boldsymbol{v}_{\text {Particle }}$ can be found by the following:

$$
\frac{d \boldsymbol{v}_{\text {Particle }}}{d t}=\frac{\boldsymbol{F}_{m}-\boldsymbol{F}_{d}}{m}
$$

where $m$ is the mass of the superparamagnetic particle.

\subsubsection{Magnetic Force}

The magnetic force on the superparamagnetic bead can be determined using the following:

$$
\boldsymbol{F}_{m}=(\boldsymbol{m} \cdot \nabla) \boldsymbol{B}
$$

where $\boldsymbol{m}$ is the magnetic dipole moment of the bead and $\boldsymbol{B}$ is the magnetic flux density of the magnet. More information on the magnetic force on the superparamagnetic beads will be discussed in the next chapter. 


\section{Chapter 3: Magnetic Theory}

In this chapter, a background on magnetostatics and the magnetic field will be given. Any standard electromagnetism book such as references [20-28] can provide additional information.

\subsection{Magnetophoresis}

Magnetophoresis is the movement of magnetizable particles in the presence of an inhomogeneous magnetic field. Although most magnetic particles exhibit ferromagnetic behavior, many non-magnetic materials display repulsive diamagnetic behavior in the presence of a magnetic field [20, 29]. As a result, magnetophoresis can be used for the isolation, separation, mixing, pumping, and general manipulation of biological material.

\subsection{The Magnetic Field}

The magnetic field is a magnetic force field produced on the atomic level as a result of the movement of electric charges within a material. A material that produces a magnetic field is referred to as a magnet, while any material that is attracted to a magnet is considered a magnetic material. Conventionally, a magnet has both a north and south pole. Although the magnetic field is invisible, its strength and magnitude are represented by magnetic field lines, which exit the north pole and enter the south pole of the magnet. The field lines form a continuous path, running from the south pole to the north pole within the magnet. Figure 18 illustrates the magnetic field lines of a magnet through the attractive properties of iron filings to the magnet [30]. The density of the magnetic field lines indicates the strength of the magnetic field with a high density indicating a strong field. 


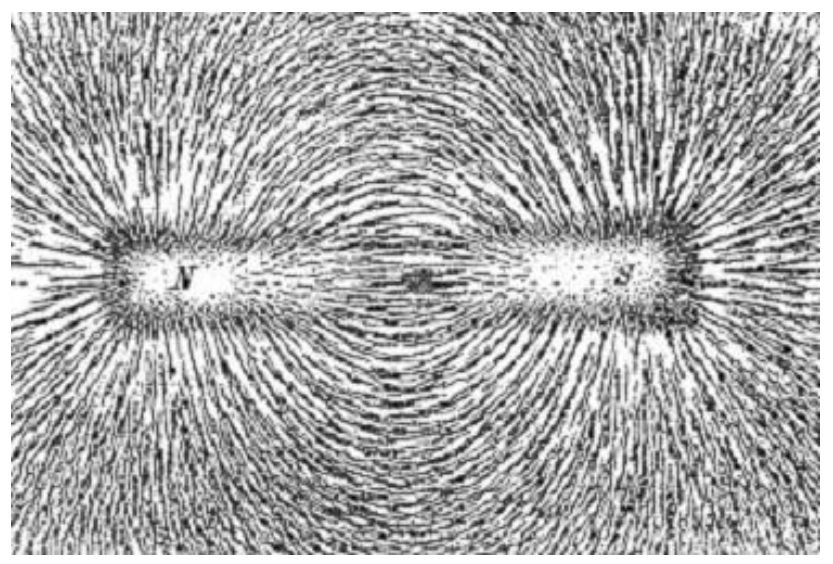

Figure 18: The Magnetic Field of a Permanent Magnet as a Result of Loose Iron Filings [30]

A magnetic field is quantified by two quantities: the magnetic flux $\phi$ and the magnetic flux density $\boldsymbol{B}$, which have units of webers $(\mathrm{Wb})$ and webers per square meter $\left(\mathrm{Wb} / \mathrm{m}^{2}\right)$ or teslas $(T)$, respectively. The magnetic flux is a measurement of the total magnetic field for a material, while the magnetic flux density is a measurement of the magnetic field per unit area of the material. A magnetic field can be either homogeneous or inhomogeneous. A homogeneous magnetic field has a magnetic flux density that is constant over a distance. In comparison, the magnetic flux density changes over a distance for an inhomogeneous magnetic field [29]. The magnetic flux density is directly related to the magnetic field intensity $\mathbf{H}$, which is independent of the material's properties, through the following constitutive equation:

$$
\boldsymbol{B}=\mu \boldsymbol{H}
$$

where $\mu$ is the permeability of the medium and has units of $H / m$. This permeability of the medium $\mu$ is specific and unique to the medium. The permeability of the medium $\mu$ can also be expressed as:

$$
\mu=\mu_{0} \mu_{r}
$$

where $\mu_{0}$ is the permeability constant of free space with a value of $4 \pi \times 10^{-7}$ henries per meter $(\mathrm{H} / \mathrm{m})$ and $\mu_{r}$ is the relative permeability of the medium. The relative permeability $\mu_{r}$ is a unitless magnetic property of the material and varies greatly. Depending on the material, $\mu_{r}$ can be either a constant or a function of $\mathbf{H}$. If $\mu_{r}$ is a constant, the material is said to be magnetically linear; a material with a relative 
permeability dependent upon the magnetic field intensity is considered a magnetically nonlinear material. The relative permeability for several materials can be seen below in Table 2 [23].

Table 2: The Relative Permeabilities of Several Materials [23]

\begin{tabular}{|c|c|}
\hline Material & $\boldsymbol{\mu}_{\mathbf{r}}$ \\
\hline Iron & 4,000 \\
\hline Permalloy & 70,000 \\
\hline Supermalloy & $1,000,000$ \\
\hline Permendur & 5,000 \\
\hline Cobalt & 600 \\
\hline Manganese-zinc ferrite & 750 \\
\hline Nickel-zinc ferrite & 650 \\
\hline Bismuth & 0.999833 \\
\hline Mercury & 0.999968 \\
\hline Copper & 0.99999906 \\
\hline Water & 0.9999912 \\
\hline Air & 1.00000037 \\
\hline Tungsten & 1.00008 \\
\hline Manganese & 1.001 \\
\hline
\end{tabular}

In free space, which is air, the large air molecules result in little effect on magnetic fields, resulting in $\mu_{r}$ being one. Under this condition:

$$
\begin{gathered}
\mu=\mu_{0} \\
\boldsymbol{B}=\mu_{0} \boldsymbol{H}
\end{gathered}
$$

\subsection{Magnetic Field Background}

The history of magnetism originates with the lonian Greek culture in 600 B.C. when the philosopher Thales of Miletus observed the attractive properties of iron to lodestone. As a result of this foundation, the term "magnetism" was derived from the ancient Greek town of Magnes where a large supply of lodestone once existed. Extensive research into magnetism did not occur until 1600 A.D. with the publication of De magnete by W. Gilbert. 
In 1819, after several years of electromagnetic progress, H.C. Oersted discovered a relationship between electricity and magnetism when a current-carrying conductor near a compass caused the compass needle to deflect. Throughout the following years, several scientists and mathematicians contributed to the electromagnetism theory. In 1855, J.C. Maxwell used some of these contributions and research he made on magnetic field lines to formulate four electromagnetic equations for free space that became known as Maxwell's Field Equations:

$$
\begin{gathered}
\nabla \cdot \boldsymbol{B}=0 \\
\nabla \times \boldsymbol{B}=\mu_{0} \boldsymbol{J}+\mu_{0} \varepsilon_{0} \frac{\partial \boldsymbol{E}}{\partial t} \\
\nabla \cdot \boldsymbol{E}=\frac{\rho}{\varepsilon_{0}} \\
\nabla \times \boldsymbol{E}=-\frac{\partial \boldsymbol{B}}{\partial t}
\end{gathered}
$$

where $\boldsymbol{B}$ is the magnetic flux density, $\mu_{0}$ is the permeability of free space, $\mathbf{J}$ is the total current density, $\varepsilon_{0}$ is the permittivity of free space, $\boldsymbol{E}$ is the electric field intensity, and $\rho$ is the total charge density. The permeability and permittivity of free space are constants with values of $4 \pi \times 10^{-7}$ henries per meter $(\mathrm{H} / \mathrm{m})$ and $8.854 \times 10^{-12}$ farads per meter $(\mathrm{F} / \mathrm{m})$, respectively.

Under static conditions, these equations become:

$$
\begin{gathered}
\nabla \cdot \boldsymbol{B}=0 \\
\nabla \times \boldsymbol{B}=\mu_{0} \boldsymbol{J} \\
\nabla \cdot \boldsymbol{E}=\frac{\rho}{\varepsilon_{0}} \\
\nabla \times \boldsymbol{E}=0
\end{gathered}
$$

Equations 3.9 and 3.10 are magnetostatics equations, and Equations 3.11 and 3.12 are electrostatics equations. For the magnetostatics equations, Equation 3.9 is known as the Law of Conservation of Magnetic Flux and states that the net magnetic flux into or out of any location is always zero. Equation 
3.10 represents Ampère's Law and states that a current will produce a magnetic flux density that rotates around it.

In vector analysis, the Helmholtz's Theorem can be applied to give characterization to a vector field through the application of the divergence and curl operations. Helmholtz's Theorem states that a continuously differentiable vector field can be uniquely determined if its divergence and curl are known everywhere within the field. From this, the vector field can be expressed through the summation:

$$
\boldsymbol{F}=\nabla f+\nabla \times \boldsymbol{G}
$$

where $\mathbf{F}$ is the continuously differentiable vector field, $f$ is a scalar field, and $\mathbf{G}$ is another vector field. If $\nabla \times \boldsymbol{F}=0$, then $\boldsymbol{F}$ can be expressed as:

$$
\boldsymbol{F}=\nabla f
$$

Vectors satisfying this condition are called irrotational vectors. If $\nabla \cdot \boldsymbol{F}=0$, then $\boldsymbol{F}$ can be expressed as:

$$
\boldsymbol{F}=\nabla \times \boldsymbol{G}
$$

Vectors that satisfy this condition are called solenoidal vectors.

As a result of Equation 3.9 and the condition associated with Equation 3.15, $\boldsymbol{B}$ is a solenoidal vector that can be expressed as:

$$
\boldsymbol{B}=\nabla \times \boldsymbol{A}
$$

where $\boldsymbol{A}$ is the magnetic vector potential. The unit for this value is webers per meter $(\mathrm{Wb} / \mathrm{m})$. When there is no current density $J$, Equation 3.9 simplifies to:

$$
\nabla \times B=0
$$

Since Equation 3.17 satisfies the condition associated with Equation $3.14, \boldsymbol{B}$ is an irrotational vector that can be expressed as:

$$
\boldsymbol{B}=-\mu_{0} \nabla \varphi
$$

where $\varphi$ is the magnetic scalar potential with units of amperes (A). 


\subsection{Magnetic Materials}

A material's magnetic properties are dependent upon the net magnetic moment $\mathbf{m}$, which has units of $A \cdot m^{2}$, that results in the presence of an external magnetic field. At the atomic level, magnetic dipole moments naturally occur as a result of both the orbital rotation and spin of electrons around the nucleus. Typically the random movement of electrons causes the magnetic dipole moments to cancel out, resulting in no net magnetic moment and thus no magnetic effect; however, permanent magnets are created when the material's dipoles permanently align to produce a net magnetic moment. The magnetic susceptibility $X$ quantifies the tendency of a material to form magnetic dipoles. It is a unitless scalar related to the relative permeability $\mu_{\mathrm{r}}$ by:

$$
X=\mu_{r}-1
$$

The magnetic susceptibility of various materials can be seen below in Table 3 [25].

Table 3: The Magnetic Susceptibility of Various Materials [25]

\begin{tabular}{|c|c|}
\hline Material & Magnetic Susceptibility $\boldsymbol{X}$ \\
\hline Bismuth & $-17.6 \times 10^{-5}$ \\
\hline Silver & $-2.4 \times 10^{-5}$ \\
\hline Copper & $-0.88 \times 10^{-5}$ \\
\hline Water & $-0.90 \times 10^{-5}$ \\
\hline Carbon Dioxide & $-1.2 \times 10^{-5}$ \\
\hline Oxygen & $0.19 \times 10^{-5}$ \\
\hline Sodium & $0.85 \times 10^{-5}$ \\
\hline Aluminum & $2.3 \times 10^{-5}$ \\
\hline Tungsten & $7.8 \times 10^{-5}$ \\
\hline Gadolinium & $48000 \times 10^{-5}$ \\
\hline Iron & $30 \times 10^{3}$ \\
\hline Iron-Nickel & $80-300 \times 10^{3}$ \\
\hline
\end{tabular}

Using Equations 3.2 and 3.19, Equation 3.1 can be expressed as:

$$
\boldsymbol{B}=\mu_{0}(1+X) \boldsymbol{H}
$$


In the presence of a magnetic field, the magnetic effect on a material's dipoles results in a change in the total magnetic flux field both inside and outside of the magnetic material. When this occurs, the material is said to be "magnetized." In materials with no natural net magnetic moment, the total magnetic flux field can either increase or decrease in response to an applied magnetic field. For those materials that have an increase in the total magnetic flux field, a magnetic field will force the dipoles to align parallel to the experienced field, resulting in a net magnetic dipole moment. Conversely, for those that experience a decrease, the magnetic field will cause a slight decrease in each magnetic dipole moment, resulting in an antiparallel net magnetic moment.

The magnetization vector $\boldsymbol{M}$, which is defined as the net magnetic moment per unit of volume and has units of $A / m$, is used to quantify the net magnetic dipole moment for a material. This vector is related to the magnetization current $\boldsymbol{J}_{m}$ or movement of bound charges through the following equation:

$$
J_{m}=\nabla \times \boldsymbol{M}
$$

Using Equations 3.10 and 3.19, the total current density $J$ can be expressed as:

$$
\boldsymbol{J}=\boldsymbol{J}_{m}+\boldsymbol{J}_{f}
$$

where $\boldsymbol{J}_{f}$ is the current density in free space. From this, Equation 3.6 becomes:

$$
\nabla \times \boldsymbol{B}=\mu_{0}\left(\boldsymbol{J}_{m}+\boldsymbol{J}_{f}\right)
$$

Substituting Equation 3.20 into Equation 3.22 produces the following:

$$
\nabla \times\left(\frac{\boldsymbol{B}}{\mu_{0}}-\boldsymbol{M}\right)=\boldsymbol{J}_{f}
$$

For linear and isotropic materials, the relative permeability $\mu_{r}$ is independent of the magnetic field intensity $\boldsymbol{H}$, and the $\boldsymbol{B}$ and $\boldsymbol{H}$ fields parallel each other in the same direction. As a result, $\boldsymbol{M}$ is proportional to $\boldsymbol{H}$ by:

$$
\boldsymbol{M}=X \boldsymbol{H}
$$

Using Equations 3.19 and 3.22 through 3.24, two additional equations can be expressed: 


$$
\begin{gathered}
\boldsymbol{H}=\frac{\boldsymbol{B}}{\mu_{0}}-\boldsymbol{M} \\
\nabla \times \boldsymbol{H}=\boldsymbol{J}_{f}
\end{gathered}
$$

Nonlinear magnetic materials vary slightly from Equation 3.26. Since the relative permeability $\mu_{r}$ depends on the magnetic field intensity $\boldsymbol{H}$, the relationship expressed in Equations 3.19 and 3.25 implies that the magnetization vector $\boldsymbol{M}$ also depends on $\boldsymbol{H}$. As a result, Equation 3.26 becomes:

$$
\boldsymbol{B}=\mu \boldsymbol{H}+\boldsymbol{B}_{\text {rem }}
$$

where $\boldsymbol{B}_{\text {rem }}$ is the remanent magnetic flux density with units of T. The remanent magnetic flux density $\boldsymbol{B}_{r e m}$ is the residual magnetic flux density in the magnet when there is no magnetic field intensity $\boldsymbol{H}$.

Magnetic materials can be classified into six different categories: diamagnetic, paramagnetic, ferromagnetic, antiferromagnetic, ferrimagnetic, and superparamagnetic. Diamagnetic and paramagnetic materials are linear, and ferromagnetic, antiferromagnetic, ferrimagnetic, and superparamagnetic materials are nonlinear. These categories are summarized in Table 4 [24].

Table 4: A Table Summary of the Classification of Magnetic Materials [24]

\begin{tabular}{|c|c|c|c|}
\hline Classification & Magnetic Moments & B Values & Comments \\
\hline Diamagnetic & $\boldsymbol{m}_{\text {orb }}+\boldsymbol{m}_{\text {spin }}=0$ & $\boldsymbol{B}_{\text {int }}<\boldsymbol{B}_{\text {appl }}$ & $\boldsymbol{B}_{\text {int }}=\boldsymbol{B}_{\text {appl }}$ \\
\hline Paramagnetic & $\boldsymbol{m}_{\text {orb }}+\boldsymbol{m}_{\text {spin }}=$ small & $\boldsymbol{B}_{\text {int }}>\boldsymbol{B}_{\text {appl }}$ & $\boldsymbol{B}_{\text {int }}=\boldsymbol{B}_{\text {appl }}$ \\
\hline Ferromagnetic & $\left|\boldsymbol{m}_{\text {spin }}\right| \gg\left|\boldsymbol{m}_{\text {orb }}\right|$ & $\boldsymbol{B}_{\text {int }} \gg \boldsymbol{B}_{\text {appl }}$ & Domains \\
\hline Antiferromagnetic & $\left|\boldsymbol{m}_{\text {spin }}\right| \gg\left|\boldsymbol{m}_{\text {orb }}\right|$ & $\boldsymbol{B}_{\text {int }}=\boldsymbol{B}_{\text {appl }}$ & Adjacent moments oppose \\
\hline Ferrimagnetic & $\left|\boldsymbol{m}_{\text {spin }}\right| \gg\left|\boldsymbol{m}_{\text {orb }}\right|$ & $\boldsymbol{B}_{\text {int }}>\boldsymbol{B}_{\text {appl }}$ & Adjacent moments oppose \\
\hline Superparamagnetic & $\left|\boldsymbol{m}_{\text {spin }}\right| \gg\left|\boldsymbol{m}_{\text {orb }}\right|$ & $\boldsymbol{B}_{\text {int }}>\boldsymbol{B}_{\text {appl }}$ & Nonmagnetic matrix \\
\hline
\end{tabular}

\subsubsection{Diamagnetic Materials}

When there is no magnetic field, the orbital and spin magnetic moments in diamagnetic materials naturally cancel out, preventing a net magnetic moment. In the presence of a magnetic field, the resultant force exerted on the material causes the orbital velocities of the electrons and their associated moments to slightly decrease. The spin moments remain unchanged. As a result, a small net magnetic moment is produced antiparallel to the magnetic field, causing the B-field within the material 
to reduce slightly. This causes the $\mu_{r}$ to become less than one and $X$ to become negative in value. With $\mu_{r}$ values less than one, diamagnetic materials experience a slight repulsive force from permanent magnets and are thus repelled. Examples of diamagnetic materials from Tables 2 and 3 include: bismuth, mercury, copper, water, silver, and carbon dioxide.

A state of perfect diamagnetism is produced when a material produces no net magnetic moment in the absence or presence of a magnetic field. Such materials are called superconductors and are strongly repulsed by permanent magnets because they have the following properties: $X=-1$, $\mu_{r}=0$, and $\boldsymbol{B}=0$. These properties only occur when the superconductor's temperature is below the critical temperature for the material. Above this critical temperature, the material has a value of $\mu_{r}=1.0$ and experiences a uniform B-field.

\subsubsection{Paramagnetic Materials}

The outer electron shells for paramagnetic materials are not filled, producing magnetic moments; however, the spin magnetic moments are randomly oriented. This results in the average magnetic moment being zero in the absence of an external magnetic field. When a magnetic field is present, more than half of the spin magnetic moments align parallel to the magnetic field while the rest of the spin magnetic moments and all of the orbital magnetic moments become antiparallel. A net magnetic moment is produced from the part of the spin magnetic moments that are aligned parallel to the magnetic field, producing a small increase in the B-field inside the material. As a result, the material has a $\mu_{r}$ value slightly greater than one, a positive $X$, and is slightly attracted to a permanent magnet. Air, tungsten, manganese, oxygen, sodium, and aluminum are examples of paramagnetic materials present in Tables 2 and 3. 


\subsubsection{Ferromagnetic Materials}

Similar to paramagnetic materials, the outer electron shells of ferromagnetic materials are not filled, resulting in magnetic moments. However, small regions within the material known as ferromagnetic domains align and lock in the same direction in the absence and presence of a magnetic field with thin layers of domain walls separating adjacent domains. In the absence of a magnetic field, these ferromagnetic domains are randomly oriented. When a magnetic field is applied, the domain walls of domains align with the field expand and grow, invading antiparallel domains. This movement is either reversible or irreversible depending upon the strength of the magnetic field. If the magnetic field is small, the domains can return to their original position once the magnetic field is removed. By applying large magnetic fields, the movement of ferromagnetic domains becomes irreversible and requires the application of a negative magnetic field to reverse the movement. The material can become saturated if the magnetic field increases enough to create a single ferromagnetic domain. At this point, any further increase in the magnetic field will have no effect on its magnetization. Ferromagnetic materials are often used as permanent magnets. They have $\mu_{r}$ values much greater than one and large positive $X$ values. The relative permeabilities for ferromagnetic materials are only constant for small ranges of the magnetic intensity field; for the rest of the range, the relative permeabilities depend on the magnetic intensity field. Examples of ferromagnetic materials include iron, iron-nickel, permalloy, supermalloy, and permendur.

The magnetization of ferromagnetic materials is graphically depicted by a hysteresis loop, which shows the relationship between $\mathbf{B}$ and $\mathbf{H}$ within the material. When a ferromagnetic material is not magnetized, it has no $\mathbf{B}$ or $\mathbf{H}$-fields, placing it at the origin of the $\mathbf{B}$ versus $\mathbf{H}$ plot. As a magnetic field is applied, its magnetization progresses linearly toward the maximum $\mathbf{H}$-field, represented by $\alpha$ in Figure 19 , until the saturation level is achieved. Once the maximum $\mathbf{H}$-field is achieved, the material cannot be magnetized any further. As the magnetic field is reduced, the material follows the hysteresis curve from 
$\alpha$ towards $\alpha^{\prime}$. When the $\mathbf{H}$-field is equal to zero, the material possesses remanent magnetization $\boldsymbol{B}_{\text {rem }}$ $\left(B_{r}\right)$. Conversely, when the B-field is equal to zero, the material achieves the coercive field intensity represented by $-\mathrm{H}_{0}$. From $\alpha^{\prime}$, the application of a positive magnetic field will return the material to $\alpha$ through the points labeled $-\mathrm{B}_{\mathrm{r}}$ and $\mathrm{H}_{0}$, resulting in a loss of energy called hysteresis loss. Ferromagnetic materials that are considered "soft" have narrow hysteresis curves while "hard" materials have wide ones [25].

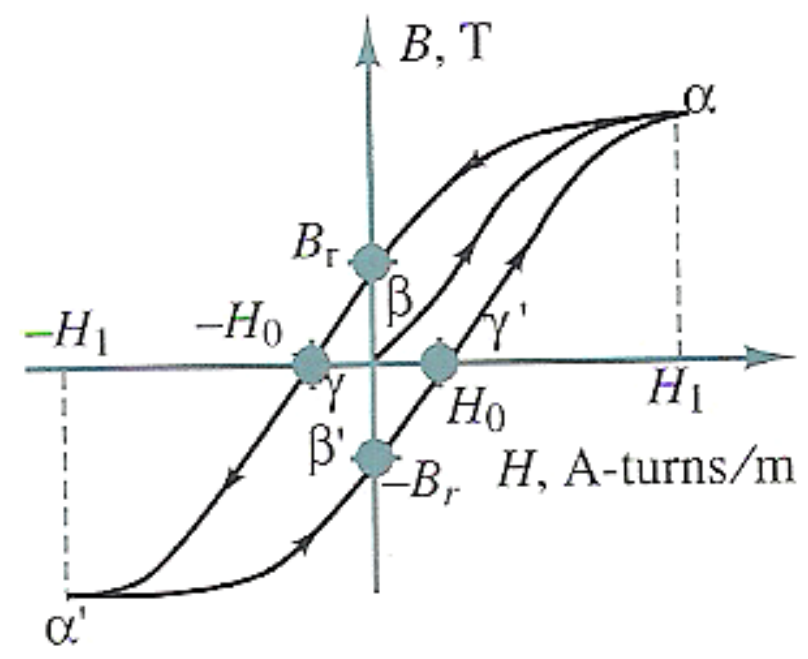

Figure 19: The Hysteresis Curve [25]

Like diamagnetic materials, ferromagnetic materials have a temperature point above which they do not behave like ferromagnetic materials any longer. This point is called the Currie temperature. Above this temperature, ferromagnetic materials lose their magnetization and behave like paramagnetic materials.

\subsubsection{Antiferromagnetic Materials}

Antiferromagnetic materials have atomic structures similar to ferromagnetic materials: their outer electron shells are not filled. As with ferromagnetic materials, this results in magnetic moments. However, the magnetic moments of adjacent atoms align antiparallel to the magnetic field as a result of 
interaction forces between atoms. This results in no net magnetic moment for the material, and a $\mu_{r}$ value of about one.

\subsubsection{Ferrimagnetic Materials}

Ferrimagnetic materials also have outer electron shells that are not filled. Like antiferromagnetic materials, the magnetic moments align antiparallel to the magnetic field. However, for ferrimagnetic materials, the magnetic moments are not equal in magnitude and thus do not cancel out. Instead, a net magnetic moment is produced for the material. Manganese-zinc ferrite and nickel-zinc ferrite are examples of ferrimagnetic materials.

\subsubsection{Superparamagnetic Materials}

Superparamagnetic materials are ferromagnetic particles surrounded by a dielectric medium. This medium allows each particle to be independently magnetized. Superparamagnetic materials are only magnetized in the presence of a magnetic field and become demagnetized when the field is removed. When a magnetic field is applied, all of the domains within the material align with the magnetic field as depicted in Figure 20 [30].

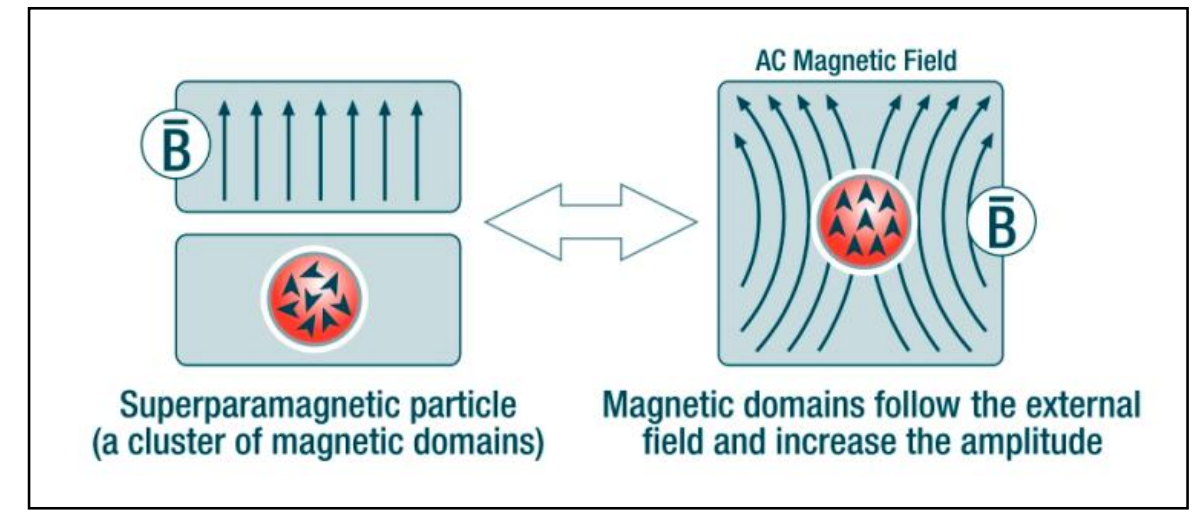

Figure 20: An Illustration of the Alignment of Magnetic Domains in a Magnetic Field [30]

As a magnetically nonlinear material, superparamagnetic materials have hysteresis curves. Superparamagnetic materials magnetize rapidly, resulting in a brief period of linearity before the 
material becomes saturated. As can be seen in the magnetic field intensity plot for superparamagnetic materials given in Figure 21, superparamagnetic beads can be treated as linear materials for small external magnetic fields [31]. They can be treated as permanent magnets above $0.5 \mathrm{~T}$.

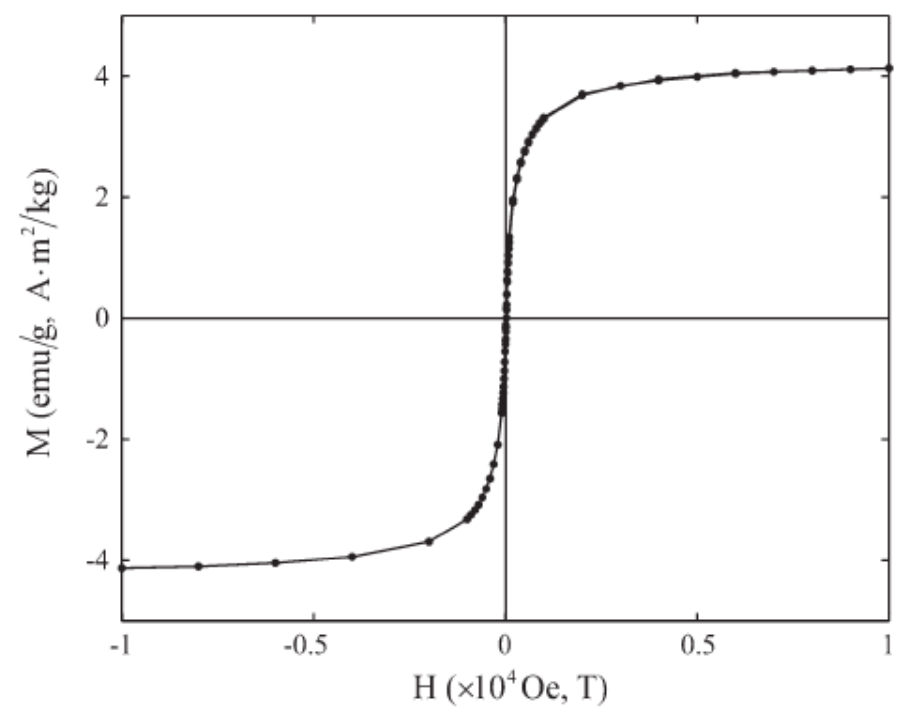

Figure 21: Magnetic Field Intensity Plot for Superparamagnetic Materials [31]

Figure 22 shows the magnetization versus magnetic field intensity plot for superparamagnetic materials for a magnetic field ranging in magnitude from -3.5 to $3.5 \mathrm{mT}$ [31].

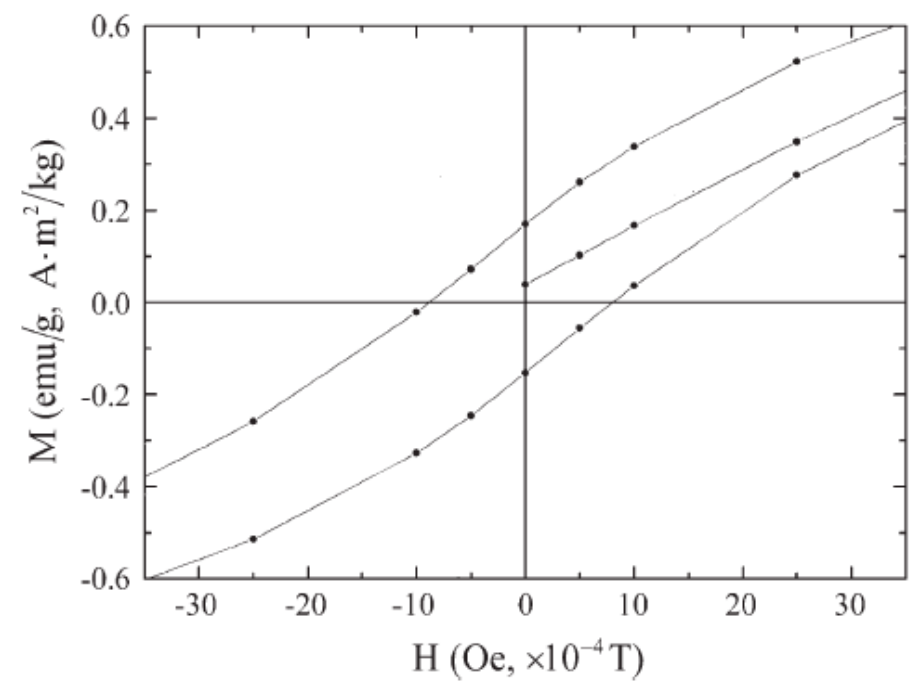

Figure 22: Magnetic Field Intensity Plot for Superparamagnetic Materials for a Range of -3.5 to $3.5 \mathrm{mT}$ [31] 
Superparamagnetic materials are used for various applications. For data-storage, the dielectric medium is frequently a thin film or tape that can store information as the superparamagnetic material rapidly changes the tape's magnetic state. Superparamagnetic beads can range in size from nanometers to a few micrometers and typically have a polystyrene matrix serving as the dielectric medium. The polystyrene coating enables covalent bonding to carboxyl or amino groups, which allow for antibody attachment in biological applications. Iron oxide is a common superparamagnetic material used for superparamagnetic beads.

\subsection{Magnetophoresis of a Superparamagnetic Bead}

According to Thomas B. Jones's book Electromechanics of Particles, the scalar potential outside and inside of a sphere, respectively, is:

$$
\begin{gathered}
\varphi_{1}(r, \theta)=-H_{0} r \cos \theta+\frac{X \cos \theta}{r^{2}}, r>R \\
\varphi_{2}(r, \theta)=-Y r \cos \theta, r>R
\end{gathered}
$$

where $H_{0}$ is the externally applied magnetic field, the subscripts 1 and 2 refer to the fluid and sphere, respectively, $R$ is the radius of the sphere, and $\mathrm{X}$ and $\mathrm{Y}$ are constants [20]. To determine these constants, two boundary conditions at the particle's surface can be applied. These conditions state that the scalar potential and magnetic flux density must be continuous between the particle and surrounding fluid and can be expressed as:

$$
\begin{gathered}
\varphi_{1}(r=R, \theta)=\varphi_{2}(r=R, \theta) \\
\mu_{1} H_{r 1}=\mu_{0}\left(H_{r 2}+M_{r 2}\right), r>R
\end{gathered}
$$

where $H_{r 1}=-\partial \varphi_{1} / \partial r$ and $H_{r 2}=-\partial 2 / \partial r$. By applying Equations 3.31 and 3.32 to Equations 3.29 and 3.30, $\mathrm{X}$ and $\mathrm{Y}$ are determined to be:

$$
X=\frac{\mu_{0}-\mu_{1}}{\mu_{0}+2 \mu_{1}} R^{3} H_{0}+\frac{\mu_{0}}{\mu_{0}+2 \mu_{1}} R^{3} M_{2}
$$




$$
Y=\frac{3 \mu_{1}}{\mu_{0}+2 \mu_{1}} H_{0}-\frac{\mu_{0}}{\mu_{0}+2 \mu_{1}} M_{2}
$$

where $\mu_{0}$ is the permeability constant of free space and $\mu_{1}$ is the magnetic permability of the fluid. The effective magnetic dipole moment $\boldsymbol{m}_{\text {eff }}$ for the sphere as a result of the applied magnetic field is related to the scalar potential of the dipole by:

$$
\varphi_{\text {dipole }}=\frac{m_{e f f} \cos \theta}{4 \pi r}
$$

As a result of Equations 3.29, 3.33, and 3.35, $\boldsymbol{m}_{\text {eff }}$ becomes:

$$
\boldsymbol{m}_{e f f}=4 \pi X=4 \pi R^{3}\left[\frac{\mu_{0}-\mu_{1}}{\mu_{0}+2 \mu_{1}} \boldsymbol{H}_{0}+\frac{\mu_{0}}{\mu_{0}+2 \mu_{1}} \boldsymbol{M}_{2}\right]
$$

The strength of the sphere's magnetization is given by the Clausius-Mossotti function, which is:

$$
K=\frac{\mu_{2}-\mu_{1}}{\mu_{2}+2 \mu_{1}}
$$

Where $\mu_{1}$ and $\mu_{2}$ are the magnetic permeabilities of the fluid and sphere, respectively. The sign of the Clausius-Mossotti function indicates the direction of the superparamagnetic beads in relation to the external magnetic field. When this function is positive, the beads are attracted to the higher and repulsed by the lower magnetic field intensities. Conversely, the beads are repulsed by the higher and attracted to the lower magnetic field intensities if the function is negative. Using Equation 3.37, the effective magnetic dipole moment can be transformed into:

$$
\boldsymbol{m}_{e f f}=4 \pi R^{3} K\left(\mu_{2}, \mu_{1}\right) \boldsymbol{H}
$$

The force exerted on a magnetic dipole by the magnetic force is:

$$
\boldsymbol{F}_{\text {dipole }}=\mu_{1} \boldsymbol{m}_{e f f} \cdot \nabla \boldsymbol{H}_{0}
$$

By inserting Equation 3.38 into Equation 3.39, the magnetophoretic force becomes:

$$
\boldsymbol{F}_{M A P}=2 \pi \mu_{1} R^{3} K\left(\mu_{2}, \mu_{1}\right) \cdot \nabla \boldsymbol{H}_{0}^{2}
$$

Above $0.5 \mathrm{~T}$ and outside the linear range for superparamagnetic beads, the magnetophoretic force is: 


$$
\boldsymbol{F}_{M A P}=2 \pi \mu_{1} R^{3}\left[\frac{\mu_{0}-\mu_{1}}{\mu_{0}+2 \mu_{1}} \nabla H_{0}^{2}+\frac{2 \mu_{0}}{\mu_{0}+2 \mu_{1}} \boldsymbol{M}_{2}\left(\boldsymbol{H}_{2}\right)\right] \cdot \nabla \boldsymbol{H}_{0}
$$

where $\boldsymbol{H}_{2}$ is a function of $\boldsymbol{H}_{0}$.

The force from Equation 3.40 can also be expressed as:

$$
\boldsymbol{F}=\frac{V \Delta X}{\mu_{0}}(\boldsymbol{B} \cdot \nabla) \boldsymbol{B}
$$

where $\Delta X$ is the difference between the magnetic susceptibilities of the bead and fluid and $V$ is the volume of the bead. Neglecting the relative permeability of the fluid, Equation 3.42 can be expressed as:

$$
\boldsymbol{F}=\frac{V X_{\text {bead }}}{\mu_{0}}(\boldsymbol{B} \cdot \nabla) \boldsymbol{B}
$$

Once a superparamagnetic bead becomes magnetized, it will have a magnetic effect on adjacent beads. To account for this additional effect, the magnetization of the bead can be added to Equation 3.43 , resulting in:

$$
\boldsymbol{F}=\boldsymbol{M} \cdot \nabla \mathbf{B}+\frac{V X_{b e a d}}{\mu_{0}}(\boldsymbol{B} \cdot \nabla) \boldsymbol{B}
$$

Equations 3.43 and 3.44 will be used for modeling superparamagnetic beads in $\mathrm{COMSOL}^{\circledR}$. 


\section{Chapter 4: COMSOL ${ }^{\circledR}$ Modeling}

Two different two-dimensional models were created for a magnetic separator system based on the one discussed in Section 1.5. The first model, which will be called the Particle Motion Model, has many limitations that ultimately resulted in the creation of a second model, which will be referred to as the Mixture Model. Both models will be discussed in this chapter.

\subsection{Implementing the Particle Motion Model}

The Particle Motion Model is similar to the particle model presented in Section 1.4. However, unlike the previous model, this model couples the transient Moving Mesh ALE, Incompressible NavierStokes, and Plane Strain modes with the static Magnetostatics mode. Through this implementation, forces placed on a particle in a microfluidic channel are taken into account in the Plane Strain mode. The geometry, physics modes, and mesh will be discussed before the presentation of the final solution in the next chapter. More information on these modes can be found in references [32-36].

\subsubsection{Geometry}

A simple geometry of four subdomains and 17 boundaries is used for this model and consists of a single particle within a fluidic channel, an adjacent permanent magnet, and a defined area of air surrounding the system. This area of air is necessary for calculating and displaying the magnetic field of the permanent magnet using the Magnetostatics mode. The single magnetic particle has a radius of $6 e-6$ $\mathrm{m}$, and the channel has a height and width of $2 \mathrm{~mm}$ and $2 \mathrm{~cm}$, respectively. The static permanent magnet, which rests on top of the fluidic channel, is $8 \mathrm{~mm}$ in width and $1 \mathrm{~cm}$ in height. The air surrounding the system has large dimensions to ensure that the magnetic field for all locations through the magnet and channel can be calculated and visualized. For this model, the air was arbitrarily given dimensions of $3.2 \mathrm{~cm}$ by $2.5 \mathrm{~cm}$. Figure 23 is an image of the basic geometry of the model. 


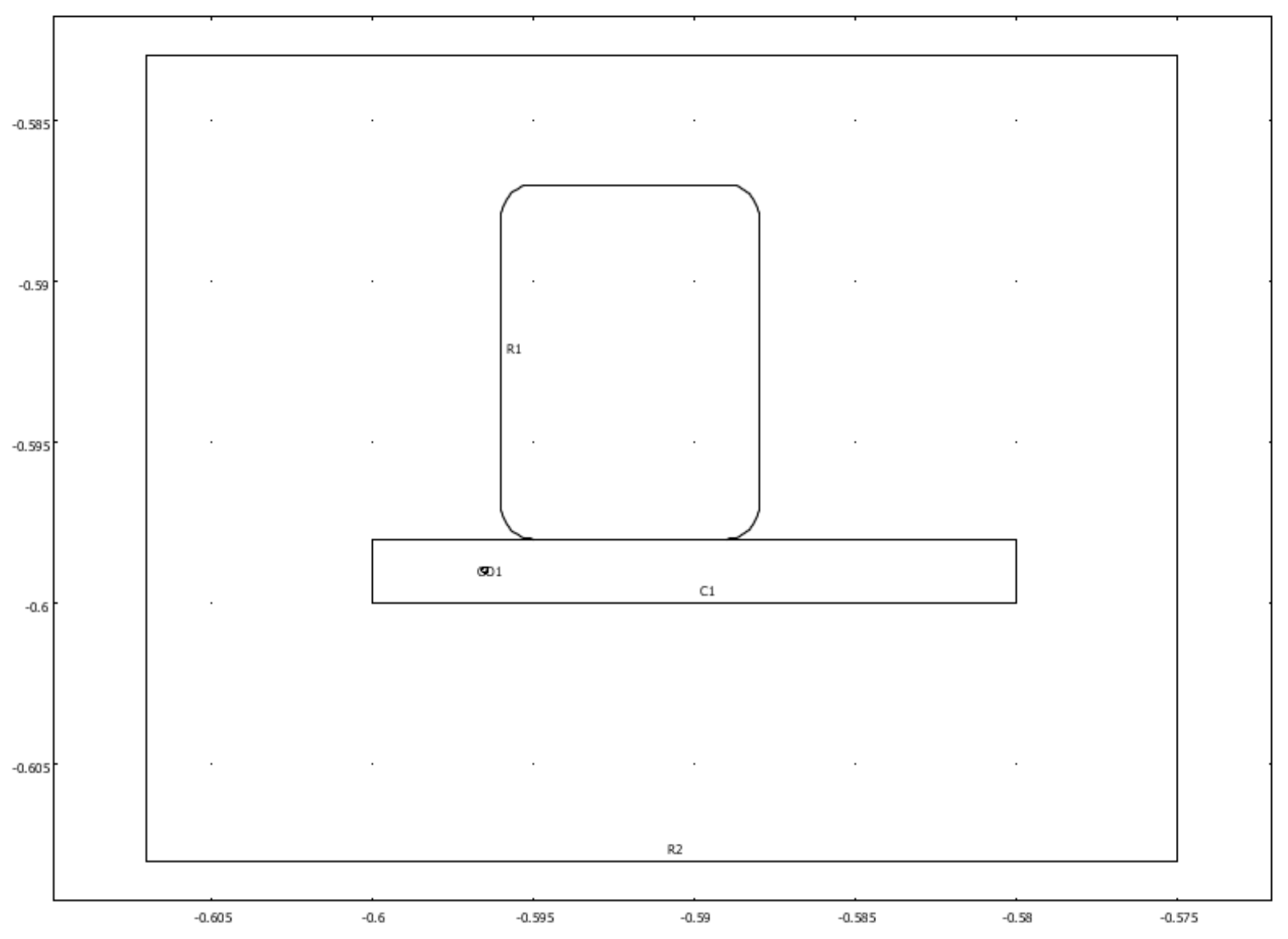

Figure 23: The Geometry of the Particle Motion Model Consisting of a Rectangular Magnet, Thin Fluidic Channel, a Small Particle, and Surrounding Air

\subsubsection{The Moving Mesh ALE Mode}

The Moving Mesh ALE mode, which has the spatial coordinate dependent variables $x$ and $y$, enables the creation of models where the mesh of the system moves as a result of the implemented physics. The displacement of the mesh is imposed through the Subdomain Settings with four possible options: free displacement, physics induced displacement, prescribed displacement, and no displacement on the "Mesh" tab. Since the displacement of the model is unknown for this model, all four subdomains are set for free displacement. This setting allows for the model's boundaries to be the only restriction on the movement of the mesh. The "Init" and "Element" tab, which provide the initial mesh displacements and order of the Lagrange element, are untouched from their original settings. For 
the Boundary Settings, the mesh displacement or velocity of the boundaries can be imposed in regard to a coordinate system. The boundaries of the air, permanent magnet, and fluidic channel have no mesh displacement for either the $\mathrm{x}$ or $\mathrm{y}$ direction. The boundaries of the particle, however, have a normal mesh velocity as a result of the fluid flow through the channel. Figure 24 illustrates the implementation of this boundary condition.

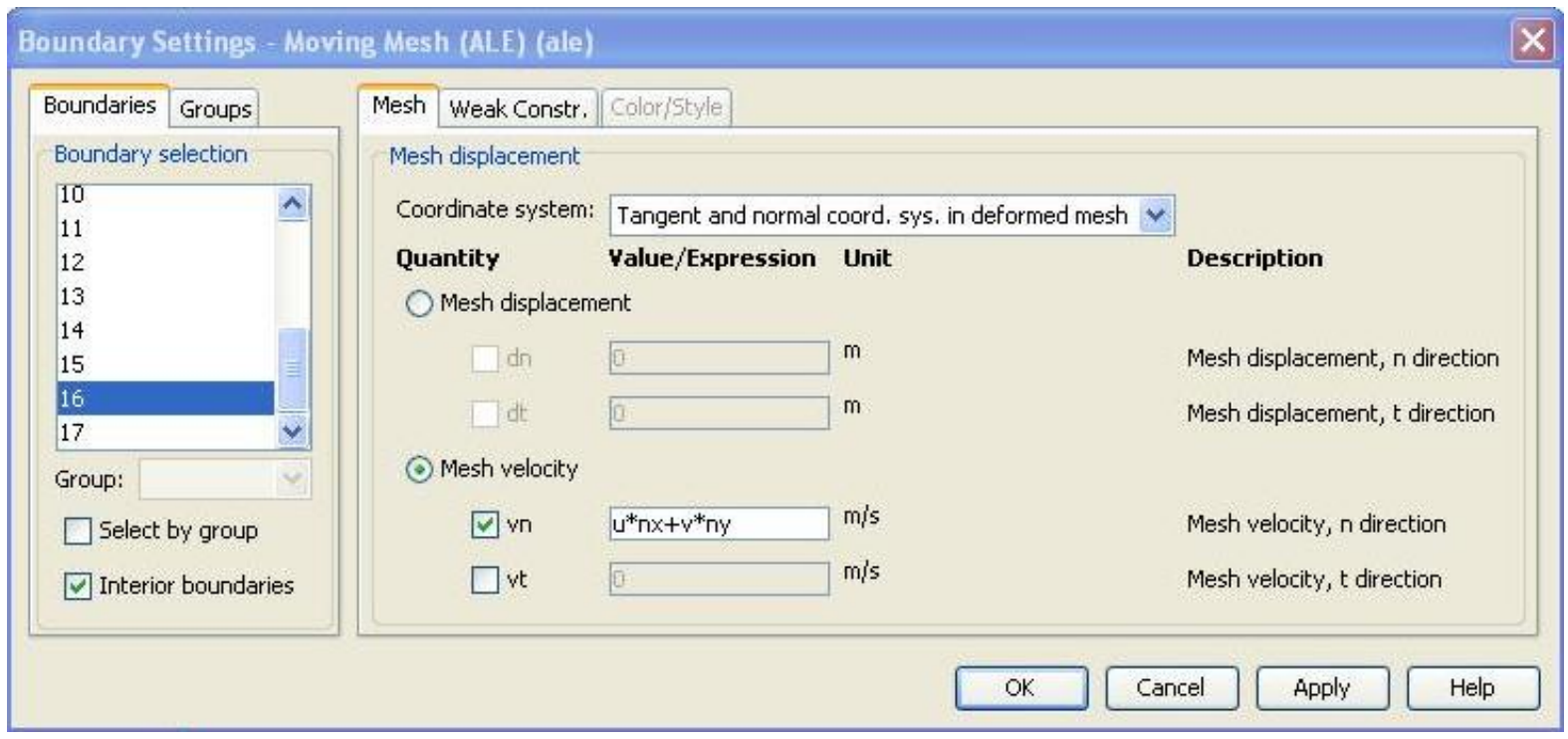

Figure 24: Illustration of the Normal Mesh Velocity Boundary Condition

In the above figure, $u$ and $v$ are the fluid velocity components from the Incompressible Navier-Stokes mode, and $\mathrm{nx}$ and ny are the normal components of the deformed mesh. Using this expression, the mesh is allowed to slip relative to the material along the boundary [33].

The properties of the physics mode enforce the remeshing and smoothing of the model. The remeshing of the model is dependent upon the amount of mesh displacement experienced by the model. Inverted mesh elements result when mesh displacement causes mesh elements to turn insideout or inverted. These elements are often unavoidable in the Moving Mesh ALE mode for models with a large amount of mesh displacement, preventing a solution for the model; however, by allowing remeshing, the amount of inverted mesh elements can often be reduced and a solution can be found. 
With free displacement Subdomain Settings, the smoothing methods, which can be either Laplace or Winslow, of the mesh displacement can be solved by one of two sets of equations. For Laplace smoothing, the model solves the following:

$$
\begin{aligned}
& \frac{\partial^{2} x}{\partial X^{2}}+\frac{\partial^{2} x}{\partial Y^{2}}=0 \\
& \frac{\partial^{2} y}{\partial X^{2}}+\frac{\partial^{2} y}{\partial Y^{2}}=0
\end{aligned}
$$

where $\mathrm{x}$ and $\mathrm{y}$ are the spatial coordinates of the spatial frame, and $\mathrm{X}$ and $\mathrm{Y}$ are the reference coordinates of the reference frame. With Winslow smoothing, the equations are reversed into the following equations:

$$
\begin{aligned}
& \frac{\partial^{2} X}{\partial x^{2}}+\frac{\partial^{2} X}{\partial y^{2}}=0 \\
& \frac{\partial^{2} Y}{\partial x^{2}}+\frac{\partial^{2} Y}{\partial y^{2}}=0
\end{aligned}
$$

The spatial frame of the model is the normal coordinate system of the entire geometry, and the reference frame describes the original system before remeshing occurs. Since the Particle Motion Model experiences considerable meshing as a result of particle movement, Winslow smoothing is used and is implemented through the following Property Settings depicted in Figure 25. 


\begin{tabular}{|c|c|c|}
\hline \multicolumn{3}{|c|}{ Application Mode Properties } \\
\hline \multirow{3}{*}{$\begin{array}{l}\text { Properties } \\
\text { Default element type: }\end{array}$} & & \\
\hline & Lagrange - Qu & atic $v$ \\
\hline & Winslow & $\checkmark$ \\
\hline $\begin{array}{l}\text { Smoothing method: } \\
\text { Analysis type: }\end{array}$ & Transient & $\checkmark$ \\
\hline \multirow{2}{*}{ Allow remeshing: } & On & $\checkmark$ \\
\hline & Frame (ale) & $v$ \\
\hline \multirow{3}{*}{$\begin{array}{l}\text { Motion relative to: } \\
\text { Weak constraints: } \\
\text { Constraint type: }\end{array}$} & Frame (mesh) & $\checkmark$ \\
\hline & On & $\checkmark$ \\
\hline & Non-ideal & $\checkmark$ \\
\hline OK & Cancel & Help \\
\hline
\end{tabular}

Figure 25: An Illustration of the Moving Mesh ALE Mode Properties for the Particle Motion Model

\subsubsection{The Incompressible Navier-Stokes Mode}

In $\mathrm{COMSOL}^{\circledR}$, the Incompressible Navier-Stokes Mode, which has the dependent variables u, v, and $p$ for the velocity and pressure of the spatial frame, solves the following Navier-Stokes equations for viscous fluids:

$$
\begin{gathered}
\rho \frac{\partial \boldsymbol{u}}{\partial t}-\nabla \cdot\left[-p \boldsymbol{I}+\eta\left(\nabla \boldsymbol{u}+(\nabla \boldsymbol{u})^{T}\right)\right]+\rho \boldsymbol{u} \cdot \nabla \boldsymbol{u}=\boldsymbol{F} \\
\nabla \cdot \boldsymbol{u}=0
\end{gathered}
$$

where $\rho$ is the density, $\boldsymbol{u}$ is the velocity, $p$ is the pressure, and $\eta$ is the dynamic viscosity of the fluid. $\boldsymbol{F}$ is the body force term, and $\boldsymbol{I}$ is the identity matrix. Under the "Physics" tab of the model's Subdomain Settings, the density, dynamic viscosity, and volumetric force of the fluid is defined. For the Particle Motion Model, only the fluidic channel is active in this mode and has Subdomain Settings internally specified for water at a temperature of $300 \mathrm{~K}$. No volumetric forces are placed on the fluid. To enable diffusion of the fluid within this mode, both the streamline and crosswind diffusion settings are selected under the "Stabilization" Subdomain Setting. 
The boundary conditions for the Incompressible Navier-Stokes mode can be divided into six categories: wall, inlet, outlet, symmetry boundary, open boundary, and stress. Walls, an inlet, and an outlet are defined as the only boundary conditions for the fluidic channel and the particle. For the channel, the two parallel lines beneath the permanent magnet are defined as walls with "No Slip" boundary conditions. As discussed in Section 2.2, fluid molecules touching the walls of the channel have a velocity of zero. The far left line of the fluidic channel is the inlet with velocity of $9 e-5 \mathrm{~m} / \mathrm{s}$, and the far right line is the outlet with a pressure of $0 \mathrm{~Pa}$. The boundaries of the particle, which will be moving during the model, have a "Moving/leaking wall" boundary condition with the velocity of the boundary equaling the rate of change of the Plane Strain mode dependent displacement variables u2 and v2.

Figure 26 illustrates the boundary condition for a boundary of the particle.

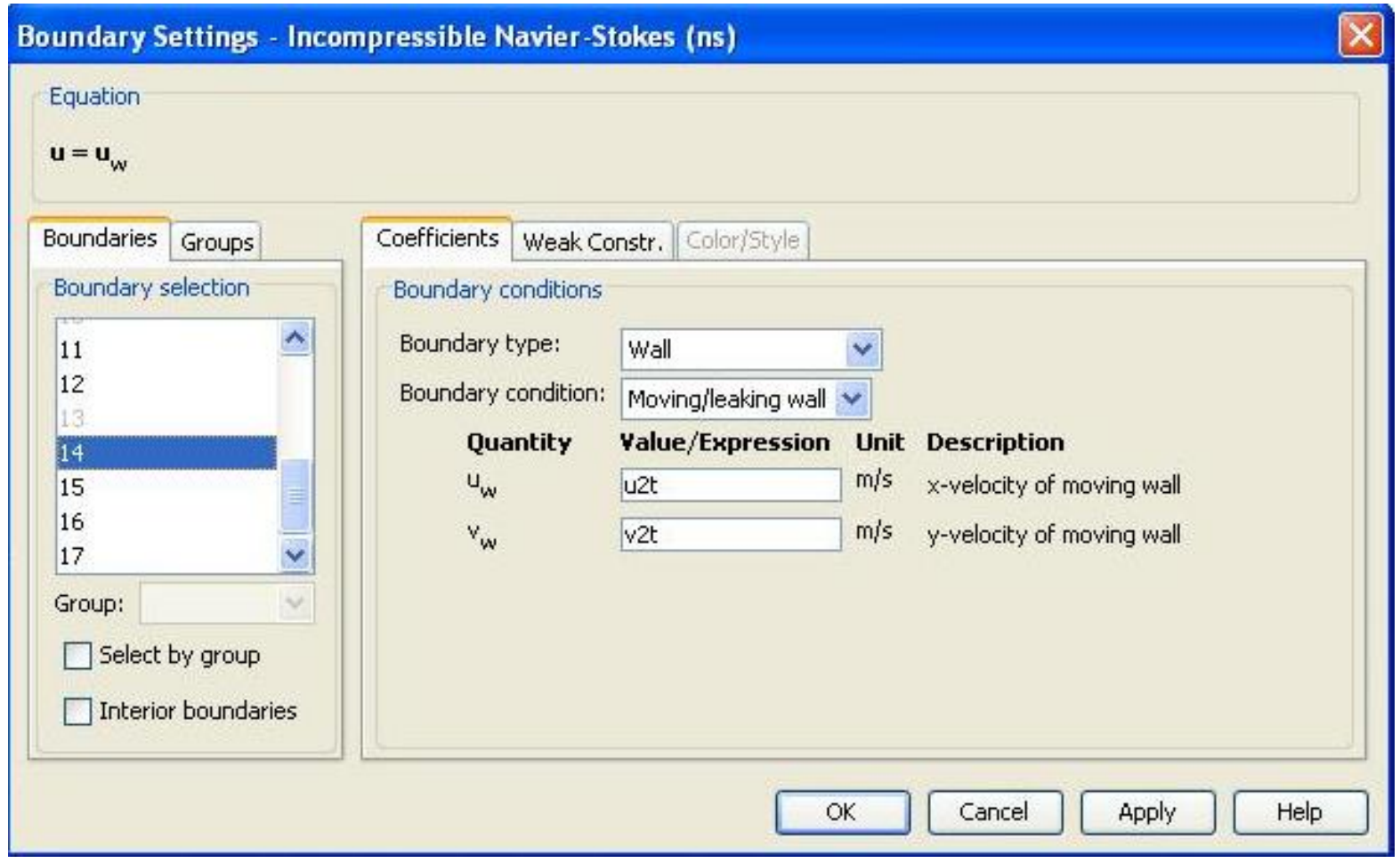

Figure 26: The Particle Boundary Conditions for the Incompressible Navier-Stokes Mode The properties of this mode remained unchanged from the default settings. 


\subsubsection{The Plane Strain Mode}

The Plane Strain mode has displacement variables $\mathrm{u} 2$ and $\mathrm{v} 2$ and calculates the strain $\varepsilon$ in the $\mathrm{x}$, $y$, and $x y$ directions using the following equations:

$$
\begin{gathered}
\varepsilon_{x}=\frac{\partial u 2}{\partial x} \\
\varepsilon_{y}=\frac{\partial v 2}{\partial y} \\
\varepsilon_{x y}=\frac{1}{2}\left(\frac{\partial u 2}{\partial y}+\frac{\partial v 2}{\partial x}\right)
\end{gathered}
$$

The Subdomain Settings of this mode define the material properties and any subdomain constraints or loads placed on the model. In the Particle Motion Model, the particle is the only active subdomain and has Subdomain Settings defined for iron (Fe) in the $\mathrm{COMSOL}^{\circledR}$ material library. These settings include a Young's modulus of $159 \mathrm{e} 9 \mathrm{~Pa}$, Poisson's ratio of 0.27 , and a density of $7860 \mathrm{~kg} / \mathrm{m}^{3}$. The thickness of the particle is defined as $100 \mathrm{e}-6 \mathrm{~m}$.

For the Boundary Settings of the particle, the "Load" tab was the only one changed from the original settings. The boundaries of the particle experienced an edge load from the fluid and magnetic field consisting of several internally defined variables. The following equations for the edge loads were used:

$$
\begin{aligned}
& F_{x}=-T_{-} x_{-} n s+d n T x_{-} q a+u n T x_{-} q a+F s L t z x_{-} q a \\
& F_{y}=-T_{-} y_{-} n s+d n T y_{-} q a+u n T y_{-} q a+F s L t z y_{-} q a
\end{aligned}
$$

where the definition of these variables are expressed in Table 5. 
Table 5: The Variable Definitions for the Forces Exerted on the Particle Motion Model

\begin{tabular}{|c|c|c|}
\hline Variable & Description & Units \\
\hline$T_{\_} x_{-} n s$ & Total Force Per Area (x-component) & $\mathrm{Pa}$ \\
\hline$T_{-} y_{-} n s$ & Total Force Per Area (y-component) & $\mathrm{Pa}$ \\
\hline$d n T x_{-} q a$ & Maxwell Surface Stress Tensor (x-component), down side of boundary & $\mathrm{Pa}$ \\
\hline$d n T y_{-} q a$ & Maxwell Surface Stress Tensor (y-component), down side of boundary & $\mathrm{Pa}$ \\
\hline$u n T x_{-} q a$ & Maxwell Surface Stress Tensor (x-component), up side of boundary & $\mathrm{Pa}$ \\
\hline$u n T y_{-} q a$ & Maxwell Surface Stress Tensor (y-component), up side of boundary & $\mathrm{Pa}$ \\
\hline FsLtzx_qa & Lorentz Surface Force Contribution (x-component) & $\mathrm{Pa}$ \\
\hline FsLtzy_qa & Lorentz Surface Force Contribution (y-component) & $\mathrm{Pa}$ \\
\hline
\end{tabular}

\subsubsection{The Magnetostatics Mode}

In the $\mathrm{COMSOL}^{\circledR}$ Magnetostatics Mode, the dependent variable is the magnetic vector potential in the z-direction $A_{z}$. For solving this application mode, the following equation is used:

$$
\boldsymbol{J}=\sigma \boldsymbol{v} \times \boldsymbol{B}+\boldsymbol{J}^{e}-\sigma \frac{\Delta V}{L}
$$

where $\boldsymbol{J}$ is the current, $\sigma$ is the electric conductivity, $\boldsymbol{v}$ is the velocity of the conductor, $\boldsymbol{J}^{e}$ is the externally generated current density, $\Delta V$ is the potential difference, and $L$ is the distance. This equation reduces for the different constitutive relations for components of the model. In the Subdomain Settings of the model, the constitutive relation and material properties for each subdomain are defined. For the Particle Motion Model, all of the subdomains are active in this mode and have different Subdomain Settings. The surrounding air had the following defined constitutive relation:

$$
\boldsymbol{B}=\mu_{0} \mu_{r} \boldsymbol{H}
$$


and the material is defined as air from the material library. Figure 27 shows the Subdomain Settings for the air subdomain.

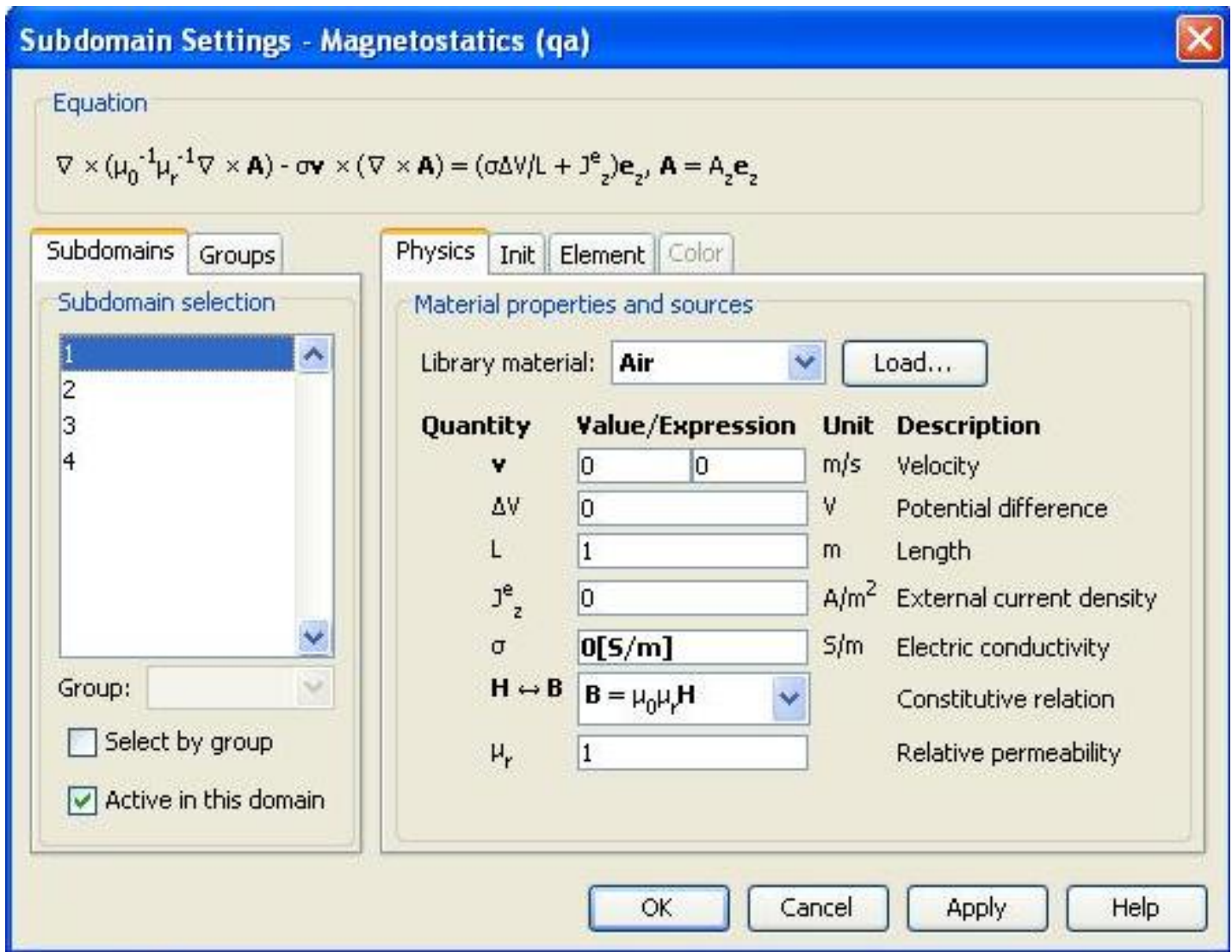

Figure 27: The Magnetostatics Mode Subdomain Settings for the Surrounding Air

The constitutive relation for the fluidic channel is:

$$
\boldsymbol{B}=\mu_{0} \boldsymbol{H}+\mu_{0} \boldsymbol{M}
$$

and the material properties are defined for water in the library mater. To couple the Incompressible Navier-Stokes mode to the Magnetostatics mode, the Incompressible Navier-Stokes velocity variables are implemented as the velocity of the channel in the Magnetostatics mode. Figure 28 illustrates the Subdomain Settings for the channel. 


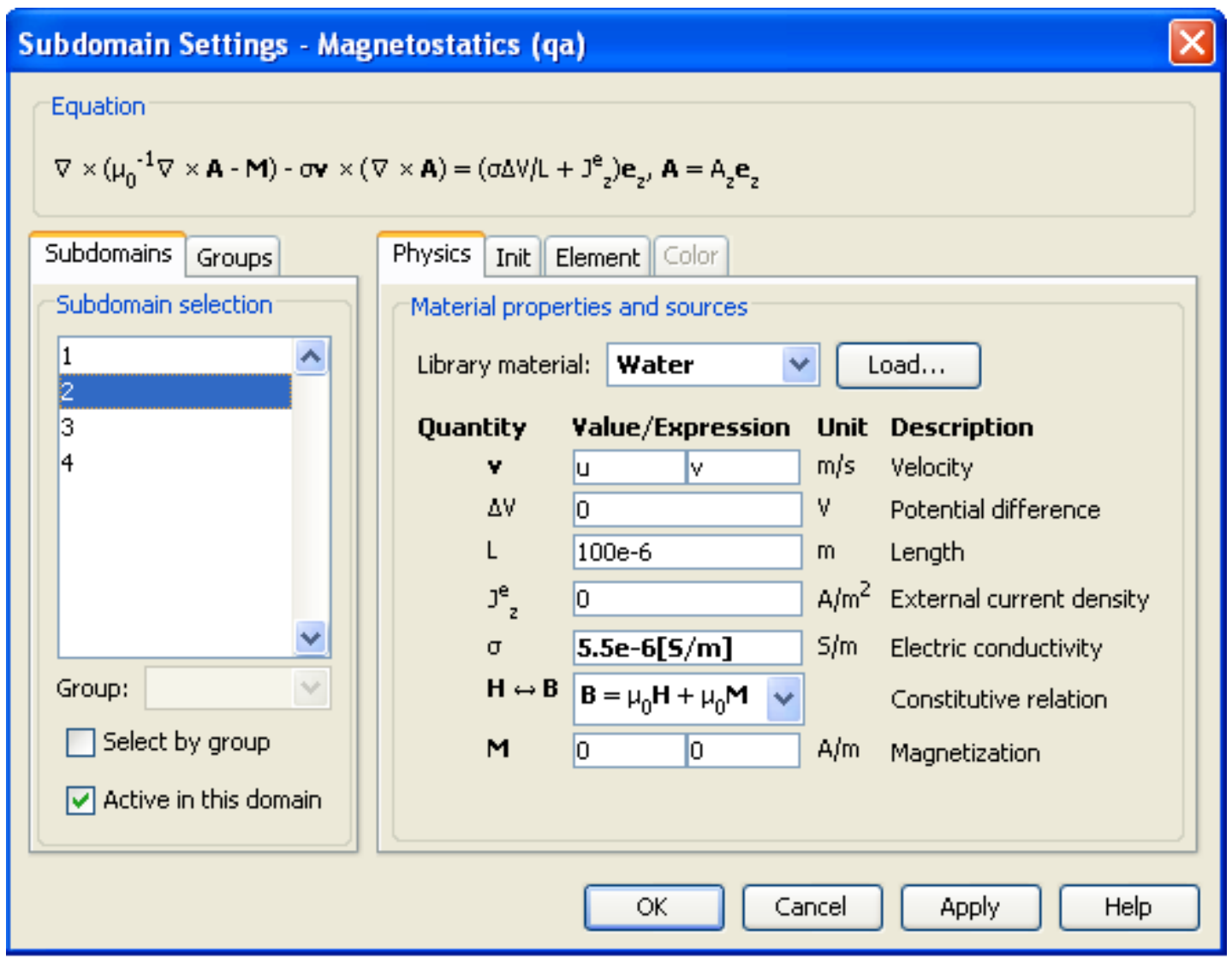

Figure 28: The Magnetostatics Mode Subdomain Settings for Water in the Particle Motion Model

The permanent magnetic has the constitutive relation:

$$
\boldsymbol{B}=\mu_{0} \mu_{r} \boldsymbol{H}+\boldsymbol{B}_{r e m}
$$

and material properties defined for iron ( $\mathrm{Fe}$ ) in the material library. The magnetic field, defined by the remanent flux density, is designated as $10 \mathrm{~T}$. The Magnetostatics properties of the permanent magnet can be seen in Figure 29. 


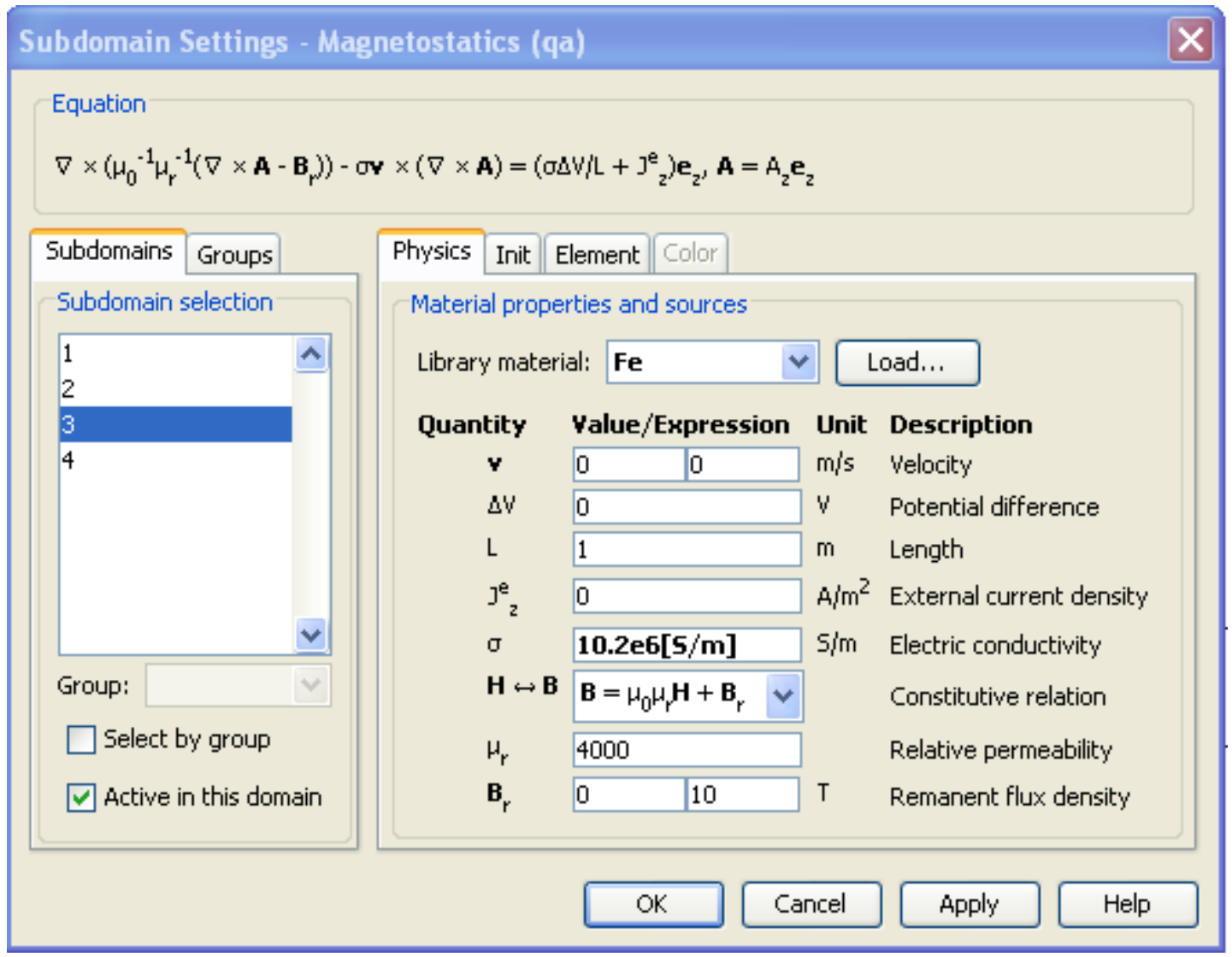

Figure 29: The Magnetostatics Mode Subdomain Settings for Permanent Magnet in the Particle Motion Model

The particle has the same constitutive relation as Equation 4.13 and magnetic properties defined for iron (Fe) in the model library. To couple the Plane Strain mode with the Magnetostatics mode, the velocity of the Magnetostatics mode for the particle is defined as the rate of change of the displacement from the Plan Strain mode. All of the particle's properties can be seen in Figure 30. 


\section{Subdomain Settings - Magnetostatics (qa)}

Equation

$\left.\nabla \times\left(\mu_{0}{ }^{-1} \mu_{r}{ }^{-1} \nabla \times \mathbf{A}\right)-\sigma \mathbf{Y} \times(\nabla \times \mathbf{A})=(\sigma \Delta V / L+]^{\mathrm{e}}{ }_{2}\right) \mathbf{e}_{2}, \mathbf{A}=\mathrm{A}_{2} \mathbf{e}_{2}$

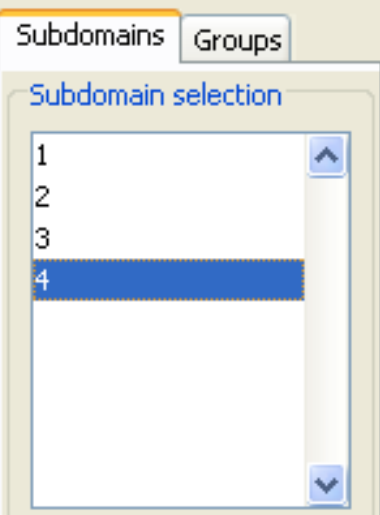

Group:

Select by group

Active in this domain \begin{tabular}{|l|lll}
\hline Physics Init Element Color & Elo \\
\hline
\end{tabular}

Material properties and sources

Library material: $\mathbf{F e}$

Load...

Quantity Value/Expression

$\mathbf{\gamma}$

$\Delta \mathrm{V}$

$\mathrm{L}$

\begin{tabular}{|l|l|}
\hline u2t & $\mathrm{v} 2 \mathrm{t}$ \\
\hline
\end{tabular}

0

$\mathrm{j}_{2}^{\mathrm{e}}$

$100 \mathrm{e}-6$

0

o $10.2 \mathrm{e} 6[\mathrm{~s} / \mathrm{m}]$

$\mathbf{H} \leftrightarrow \mathbf{B} \quad \mathbf{B}=\mu_{0} \mu_{r} \mathbf{H}$

Unit Description

mis Velocity

v Potential difference

$\mathrm{m}$ Length

Ailm ${ }^{2}$ External current density

Sim Electric conductivity

Constitutive relation

$\mu_{r}$

4000

Relative permeability

Figure 30: The Magnetostatics Mode Subdomain Settings for the Particle in the Particle Motion Model

The boundary conditions for the Magnetostatics mode of the Particle Motion Model are left at their original conditions with all of the boundaries having magnetic insulation or a magnetic vector potential of zero.

\subsubsection{The Mesh}

To avoid inverted mesh elements, the mesh for the Particle Motion Model needed to be very fine around the particle where remeshing occurs. This is accomplished by using "Free Mesh Parameters" to define a maximum element size of $5 e-5$ for the fluidic channel subdomain. Figure 31 depicts the resulting mesh for the model, which consists of 5,222 triangle mesh elements. 


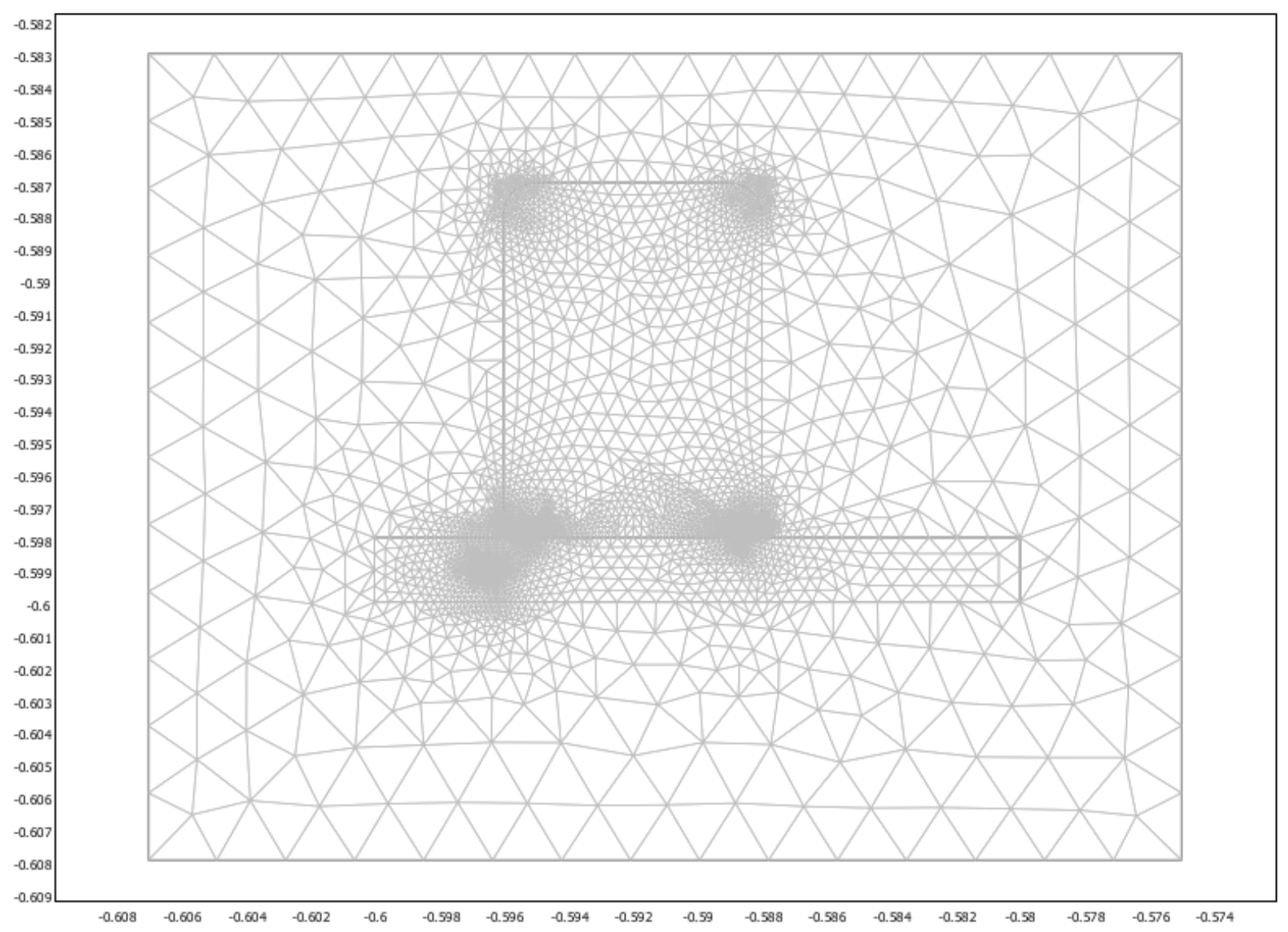

Figure 31: The Mesh of the Particle Motion Model

Finally, the model is solved for 10 seconds with a 0.01 second time step using the UMFPACK linear system solver. The solution of this model will be presented in the next chapter.

\subsection{Implementing the Mixture Model}

The Mixture Model discussed in this section provides a novel, two-dimensional simulation of superparamagnetic beads in a magnetic separator or trapping system. Unlike previous models, the Mixture Model involves the coupling of the Mixture mode with the Magnetostatics mode. This section will discuss the geometry, physics modes, and meshing used to create the Mixture Model. References [36-37] can provide more information about the used $\mathrm{COMSOL}^{\circledR}$ modes. 


\subsubsection{The Geometry}

Similar to the Particle Motion Model, the geometry of the Mixture Model consists of air surrounding the system, a channel, and a permanent magnetic. However, a circular inlet for a second fluid is located within the fluidic channel instead of a single particle. This inlet for the second fluid is concentrated with numerous beads. The surrounding air has a height and width of 2.5 and $3.2 \mathrm{~cm}$, respectively, and the permanent magnet has a height of $1.1 \mathrm{~cm}$ and a width of $8 \mathrm{~mm}$. The channel has dimensions of $2 \mathrm{~mm}$ by $2 \mathrm{~cm}$, and the inlet within the fluidic channel has a radius of 3.0e- $4 \mathrm{~m}$. Figure 32 is an image of the Mixture Model's geometry.

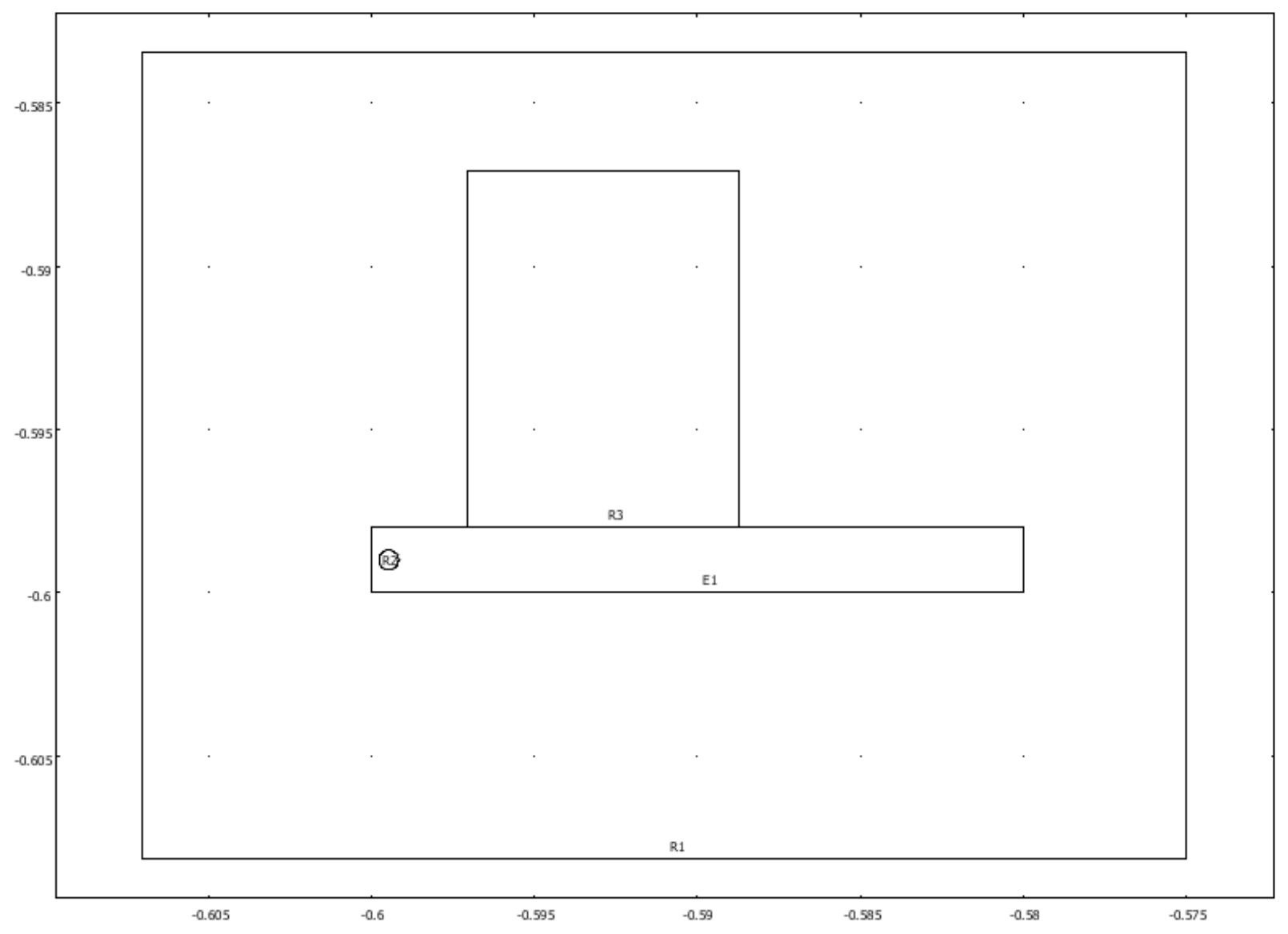

Figure 32: The Geometry of the Mixture Model Consisting of a Rectangular Magnet, Thin Fluidic Channel, a Small Circular Inlet, and Surrounding Air 


\subsubsection{The Mixture Mode}

The Mixture mode enables the monitoring of the volume fraction and velocity of two fluids in relation to one another through the dependent variables $u, v, p$, and $\Phi_{d}$, which are the velocity components, pressure, and volume fraction of the dispersed fluid of the system. This model calculates a solution using the following equation:

$$
\rho \frac{\partial \boldsymbol{u}}{\partial t}+\rho(\boldsymbol{u} \cdot \nabla) \boldsymbol{u}=-\nabla p-\nabla \cdot\left(\frac{p \Phi_{d} \rho_{d}}{\rho\left(1-\frac{\Phi_{d} \rho_{d}}{\rho}\right)}\right) \boldsymbol{u}_{\text {slip }} \boldsymbol{u}_{\text {slip }}+\nabla \cdot\left(\eta\left[\nabla \boldsymbol{u}+\nabla \boldsymbol{u}^{T}\right]\right)+\rho \boldsymbol{g}+\boldsymbol{F}
$$

where $\Phi_{d}$ is the volume fraction of the dispersed fluid, $\rho_{d}$ is the density of the dispersed fluid, $\boldsymbol{u}_{\text {slip }}$ is the relative velocity between the two fluids, $\rho$ is the mixture density, $p$ is the mixture pressure, $\eta$ is the mixture dynamic viscosity, $\boldsymbol{g}$ is the gravity vector, and $\boldsymbol{u}$ is defined by the following:

$$
\boldsymbol{u}=\left(\frac{\Phi_{c} \rho_{c} \boldsymbol{u}_{c}+\Phi_{d} \rho_{d} \boldsymbol{u}_{d}}{\rho}\right)
$$

The c subscript indicates properties for the continuous fluid, and the $d$ subscript applies to the dispersed fluid. For the Subdomain Settings for the Mixture Model, only the fluidic channel and the inlet within the channel are active in the Mixture mode. Under the "Materials" tab of the Subdomain Settings, the fluid within the channel is defined as water for the continuous phase and solid iron particles for the dispersed phase at a temperature of $300 \mathrm{~K}$. On the "Physics" tab, the iron particles are given a diameter of 1e-4 m and volumetric force components of (Ftx, Fty). These force components are defined in Table 6. 
Table 6: The Volumetric Forces Implemented on the Mixture Model

\begin{tabular}{|c|c|c|}
\hline Volumetric Force & COMSOL $^{\circledR}$ Expression & Units \\
\hline Ftx & $\mathrm{Fx}+\mathrm{F} 1$ & $\mathrm{~N} / \mathrm{m}^{3}$ \\
\hline Fty & $\mathrm{Fy}+\mathrm{F} 2$ & $\mathrm{~N} / \mathrm{m}^{3}$ \\
\hline Fx & $(A z x * A z x x+A z y * A z x y) *\left(c h i \_f f /\left(m u 0 \_q a\right)\right) * c d \_c h m m$ & $\mathrm{~N} / \mathrm{m}^{3}$ \\
\hline Fy & $(A z x * A z x y+A z y * A z y y) *\left(c h i=f f /\left(m u 0 \_q a\right)\right) * c d \_c h m m$ & $\mathrm{~N} / \mathrm{m}^{3}$ \\
\hline F1 & $\left(M x \_q a * A z x x+M y \_q a * A z x y\right) * c d \_c h m m$ & $\mathrm{~N} / \mathrm{m}^{3}$ \\
\hline F2 & $\left(M x \_q a * A z x y+M y \_q a * A z y y\right) * c d \_c h m m$ & $\mathrm{~N} / \mathrm{m}^{3}$ \\
\hline
\end{tabular}

where $\mathrm{Mx}$ _qa and My_qa are internal $\mathrm{COMSOL}^{\circledR}$ variables for magnetization, Azx and Azy are the firstorder partial derivatives of the magnetic potential in the $\mathrm{x}$ and $\mathrm{y}$ directions, Azxx and Azyy are the second-order partial derivatives of the magnetic potential in the $\mathrm{x}$ and $\mathrm{y}$ directions, Azxy is the secondorder mixed-derivative of the magnetic potential with respect to both the $\mathrm{x}$ and $\mathrm{y}$ directions, and cd_chmm is the $\mathrm{COMSOL}^{\circledR}$ variable of the dispersed volume fraction. The variables chi_ff and mu0_qa are constants; chi_ff, which is the susceptibility of the particles, is equal to 30,000 , and mu0_qa is the magnetic permeability of free space or $\mu_{0}$. These expressions are derived from Equation 3.44. In mathematical terms, Ftx and Ftz are equivalent to:

$$
\begin{aligned}
& F t x=\Phi_{d}\left[\left(M_{x} \frac{\partial^{2} A_{z}}{\partial x^{2}}+M_{y} \frac{\partial^{2} A_{z}}{\partial x \partial y}\right)+\frac{X}{\mu_{0}}\left(\frac{\partial A_{z}}{\partial x} \frac{\partial^{2} A_{z}}{\partial x^{2}}+\frac{\partial A_{z}}{\partial y} \frac{\partial^{2} A_{z}}{\partial x \partial y}\right)\right] \\
& F t x=\Phi_{d}\left[\left(M_{x} \frac{\partial^{2} A_{z}}{\partial x \partial y}+M_{y} \frac{\partial^{2} A_{z}}{\partial y^{2}}\right)+\frac{X}{\mu_{0}}\left(\frac{\partial A_{z}}{\partial x} \frac{\partial^{2} A_{z}}{\partial x \partial y}+\frac{\partial A_{z}}{\partial y} \frac{\partial^{2} A_{z}}{\partial y^{2}}\right)\right]
\end{aligned}
$$

where $\Phi_{d}$ is the volume fraction of the dispersed fluid, $M_{x}$ and $M_{y}$ are the magnetization in the $\mathrm{x}$ and $\mathrm{y}$ directions, $\frac{\partial A_{z}}{\partial x}$ and $\frac{\partial A_{z}}{\partial y}$ are the first-order partial derivatives of the magnetic potential in the $\mathrm{x}$ and $\mathrm{y}$ directions, $\frac{\partial^{2} A_{z}}{\partial x^{2}}$ and $\frac{\partial^{2} A_{z}}{\partial y^{2}}$ are the second-order partial derivations of the magnetic potential in the $\mathrm{x}$ and $\mathrm{y}$ directions, and $\frac{\partial^{2} A_{z}}{\partial x \partial y}$ is the second-order mixed-derivative with respect to both the $\mathrm{x}$ and $\mathrm{y}$ directions. For artificial diffusion, both the streamline and isotropic settings are selected for the channel and inlet 
subdomains. The only difference between the Subdomain Settings for the channel and inlet subdomains is on the "Init" tab where the initial volume fraction of the dispersed phase is equal to 0.5 .

For the boundary conditions of the model, the line on the far left of the channel is the inlet of the channel and has a pressure of $10 \mathrm{~Pa}$. On the "Disperse Phase" tab, the inlet has a "Dispersed phase flux" boundary condition with no dispersed phase or number density flux as seen in Figure 33 . This condition creates an insulation of the dispersed phase at the boundary, preventing any losses of the superparamagnetic bead fluid at the walls.

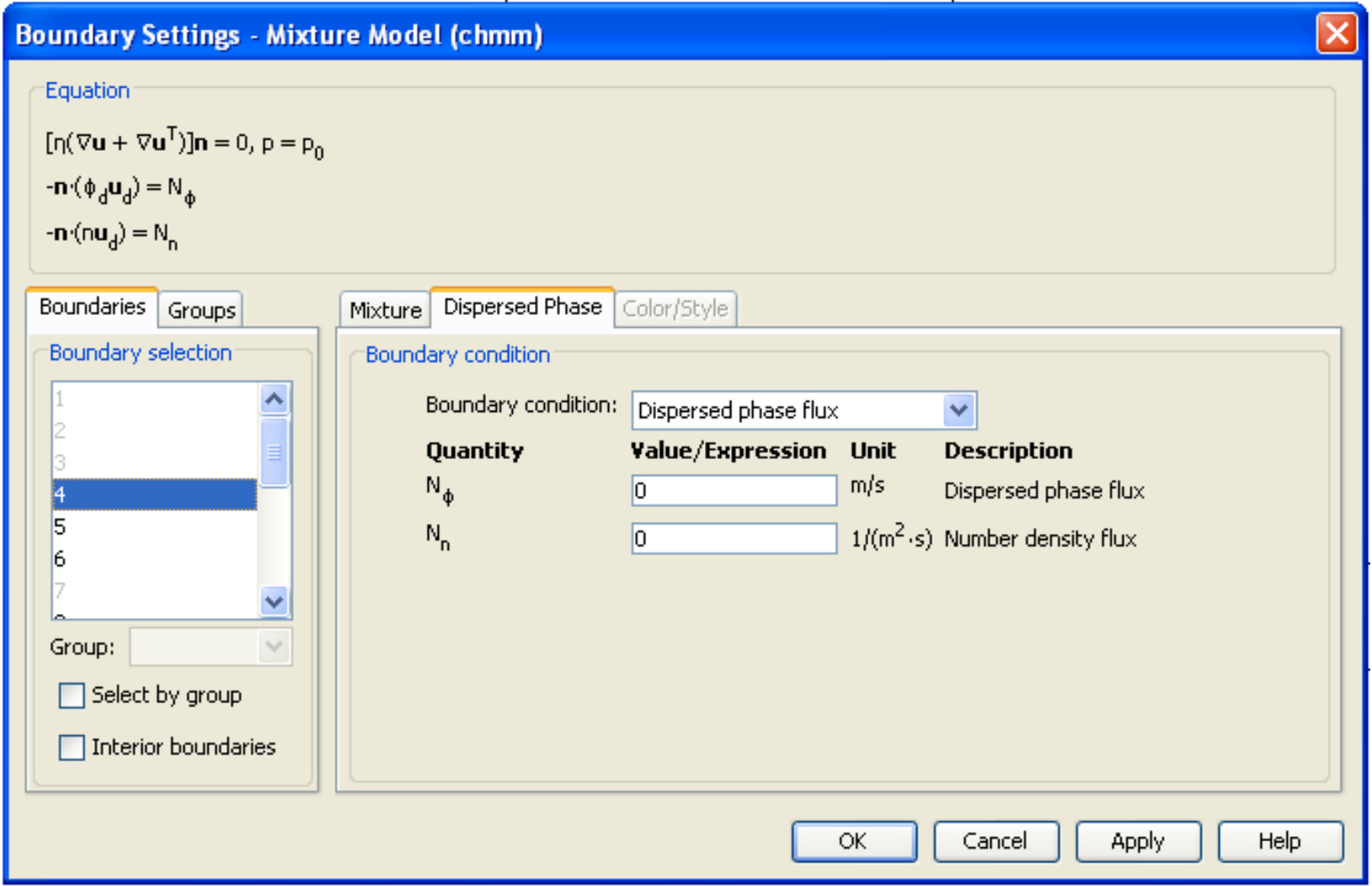

Figure 33: The Mixture Model Boundary Conditions for the Inlet

The far right vertical line of the channel is the outlet and has a pressure of $0 \mathrm{~Pa}$. For the "Disperse Phase" tab, the outlet has a "Dispersed phase outlet" boundary condition. All of the horizontal lines of the channel are no slip walls with insulation dispersed phase boundary conditions. 


\subsubsection{The Magnetostatics Mode}

The Magnetostatics mode settings for the Mixture Model are similar to the Particle Motion Model discussed in Section 4.1 with all four subdomains being active in the mode. For the Subdomain Settings, the magnetic properties of the surrounding air are implemented through the $\mathrm{COMSOL}^{\circledR}$ material library and setting the constitutive relation to Equation 4.13. The fluid within the channel is defined as water and Equation 4.14 constitutive relation. To couple the Magnetostatics mode with the Mixture mode, the Mixture mode velocity variables $u$ and $v$ are used as the velocity variables for the Magnetostatics mode of the channel fluid. All of these settings and arbitrarily defined length of $100 \mathrm{e}-6$ $\mathrm{m}$ can be seen in Figure 34 .

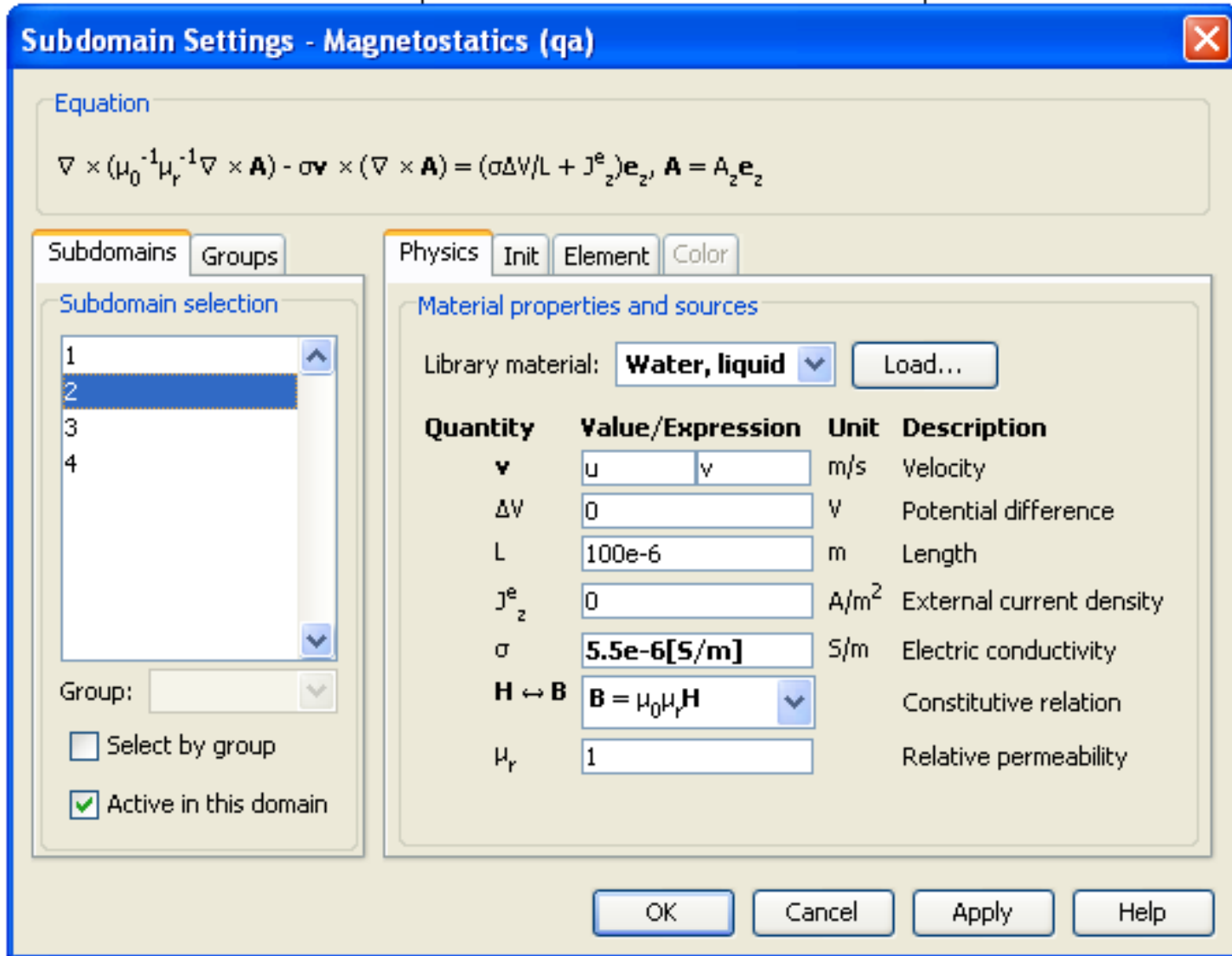

Figure 34: The Magnetostatics Mode Subdomain Settings for the Channel in the Mixture Model 
Using Equation 4.15 and the Iron material properties from the material library, the Magnetostatics mode Subdomain Settings are defined for the permanent magnet with a y-component remanent flux density of $0.5 \mathrm{~T}$. Similar to the channel fluid, the Magnetostatics mode Subdomain Settings for the second fluid inlet have a defined constitutive relation equal to Equation 4.14, coupled velocity variables $u$ and $v$, and a length of $100 e-6$. However, the magnetization for the inlet is defined by the following equations:

$$
\begin{gathered}
M x=\frac{X}{\mu_{0}}\left(\frac{\partial A_{z}}{\partial y}\right) \\
M y=-\frac{X}{\mu_{0}}\left(\frac{\partial A_{z}}{\partial x}\right)
\end{gathered}
$$

These settings are displayed in Figure 35.

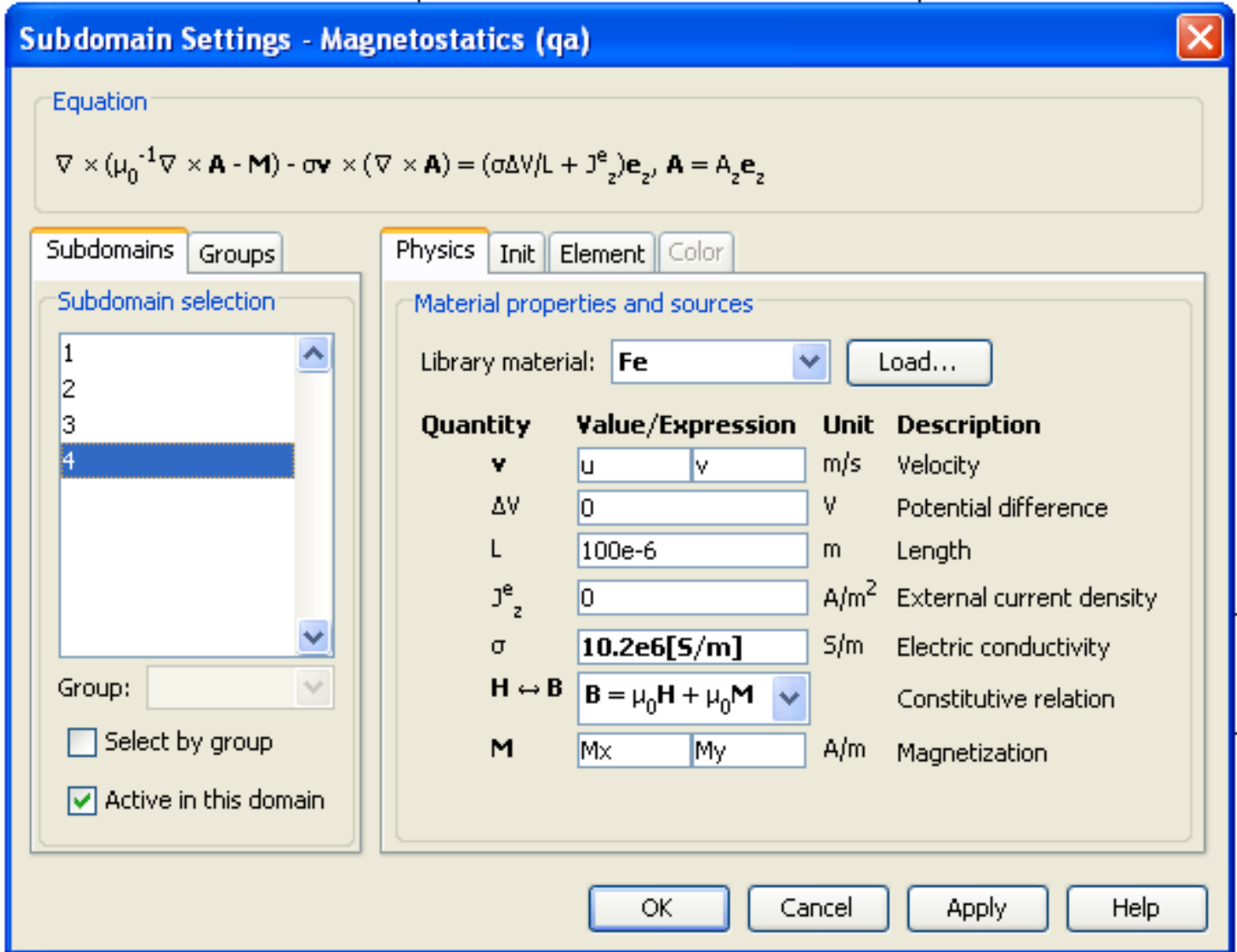

Figure 35: The Magnetostatics Mode Subdomain Settings for the Inlet of the Mixture Model 
As with the Particle Motion Model, the Magnetostatics mode Boundary Settings are not changed from the default.

\subsubsection{The Mesh}

For the Mixture Model, the mesh element sizes and concentration is important for the channel, inlet, and permanent magnet where the solution of the magnetic field has its greatest impact. To accomplish this, a maximum element size of $5.5 \mathrm{e}-3$ is defined for the channel and inlet subdomains in the Free Mesh Parameters. The resulting mesh can be seen in Figure 36.

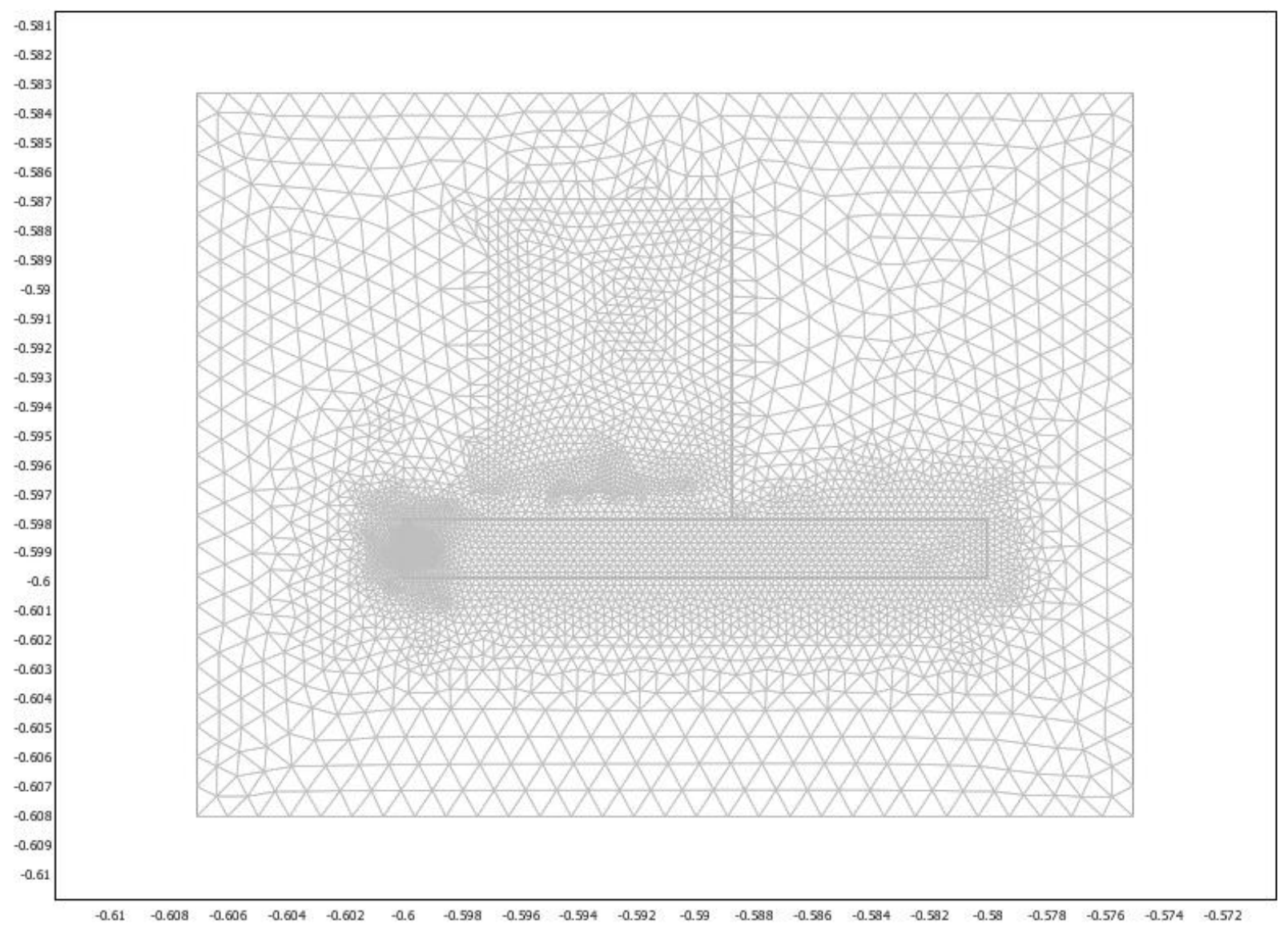

Figure 36: The Mesh for the Mixture Model

To ensure the accuracy of this mesh, a Convergence Study was performed using different "Free Mesh Settings" and documenting the maximum volume fraction beneath the left side of the permanent 
magnet at $0.10 \mathrm{~s}$ of the model's solution. The number of mesh elements was used to quantify the mesh. The results of this Convergence Study can be seen in Figure 37.

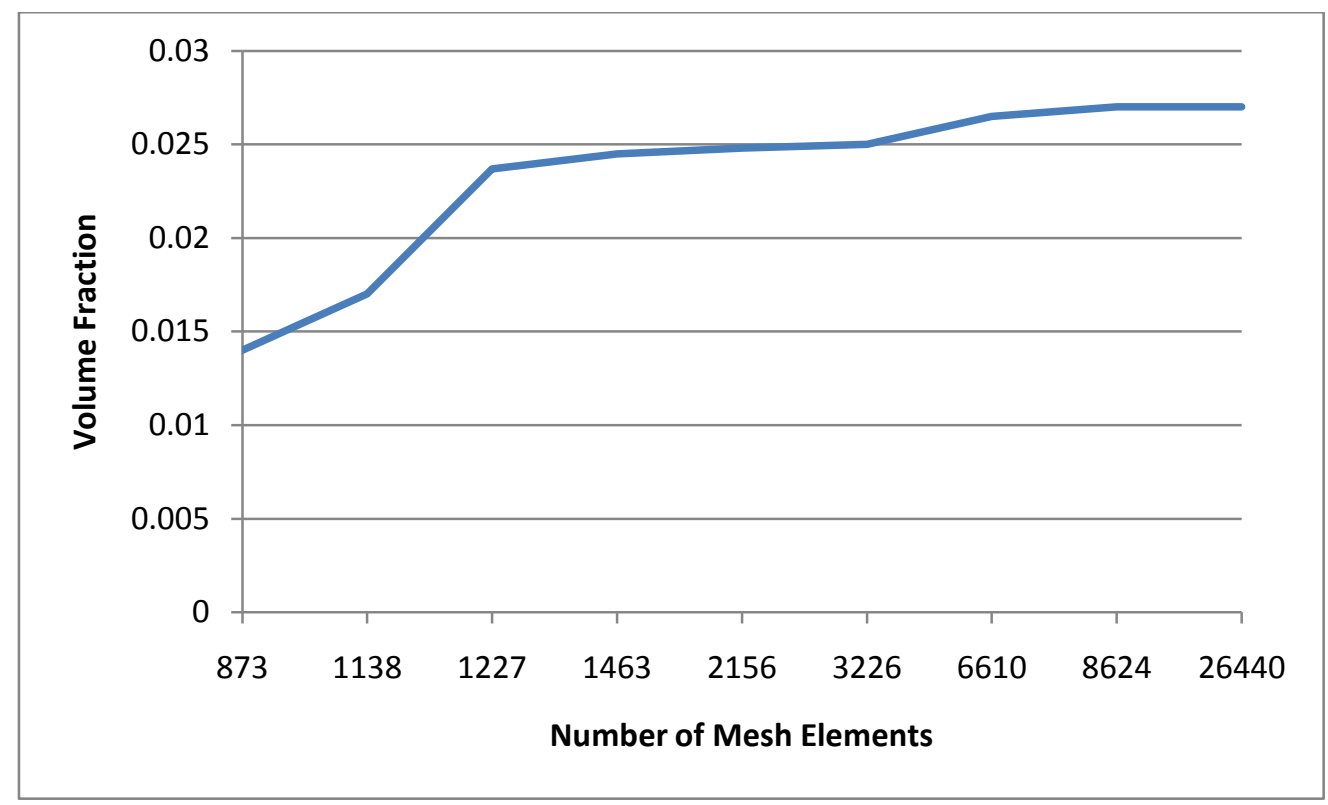

Figure 37: The Convergence Study for the Mixture Model

As Figure 37 indicates, models with over approximately 6,000 mesh elements produce consistent and accurate results. The mesh for the Mixture Model consists of 9,896 triangle mesh elements.

This model was solved for 10 seconds with 0.01 second time steps using the UMFPACK linear system solve, and the solution will be introduced in the next chapter. 


\section{Chapter 5: Modeling Results and Future Directions}

In this chapter, the results from the physics modes and settings implemented in Chapter 4 will be presented and discussed for both the Particle Motion Model and the Mixture Model. This chapter will conclude with suggestions of future directions that can be taken to model magnetic separators and superparamagnetic particles in $\mathrm{COMSOL}^{\circledR}$.

\subsection{The Particle Motion Model}

The final solution for the Particle Motion Model can be seen in Figure 38, which is a plot of the fluid's velocity for the surface of the channel and magnetic potential contour lines of the magnet.

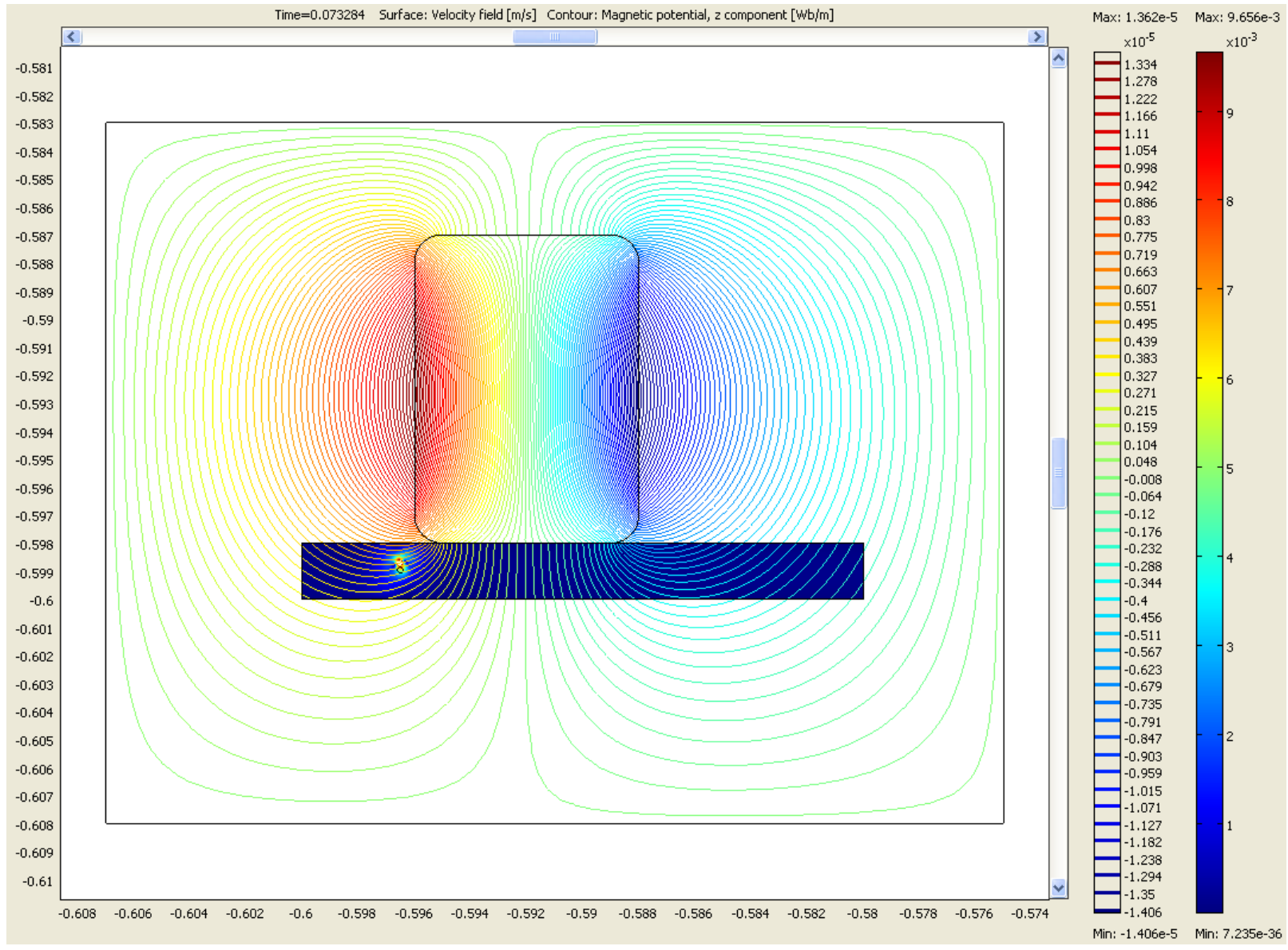

Figure 38: The Fluid Velocity Surface Plot and Magnetic Potential Contour Plot of the Particle Motion Model 
The magnetic potential contour lines of the permanent magnet indicate that the left and right sides of the magnet have the greatest magnetic potential magnitudes with opposite signs. This is expected for a two-dimensional representation of a permanent magnet. As discussed in Chapter 3, the magnetic field lines of a permanent magnet continuously travel from the south pole to the north pole of the magnet, resulting in the largest magnetic field existing where the magnetic field lines have the shortest distance to travel. For the system modeled in $\mathrm{COMSOL}^{\circledR}$, the north pole and south pole of the permanent magnet are designated as the top and bottom horizontal lines of the magnet. This results in the largest magnetic field existing on the left and right sides of the permanent magnet where the magnetic field lines have the shortest distance to travel between the poles and the smallest magnetic potential towards the middle of the magnet where the magnetic field lines have the largest distance to travel. Since the magnetic field and magnetic potential are related through Equation 3.16, the magnetic potential of the model, which can be seen more clearly in Figure 39, has results similar to the magnetic field of the magnet. The observed sign difference for the right side of the magnet is the result of Equation 3.16.

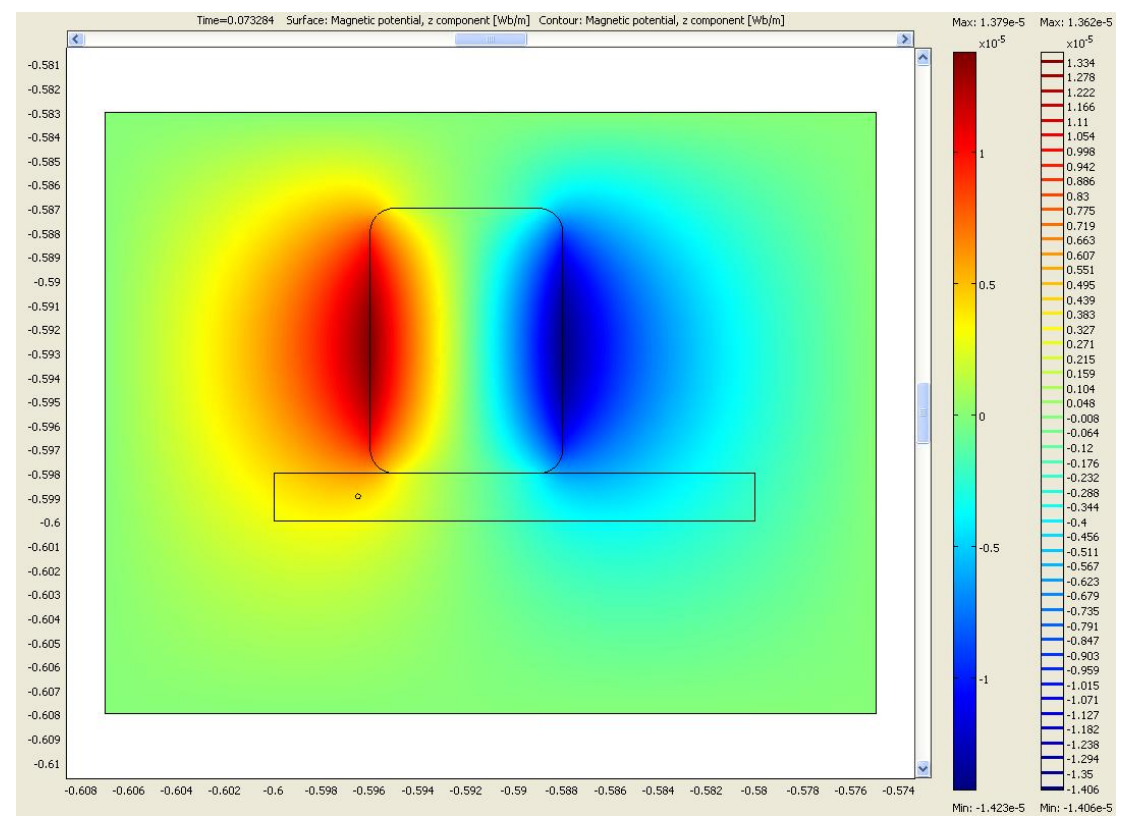

Figure 39: The Magnetic Potential of the Particle Motion Model

The magnetic field of the model can be seen in Figure 40 with a plot of the magnetic flux density. 

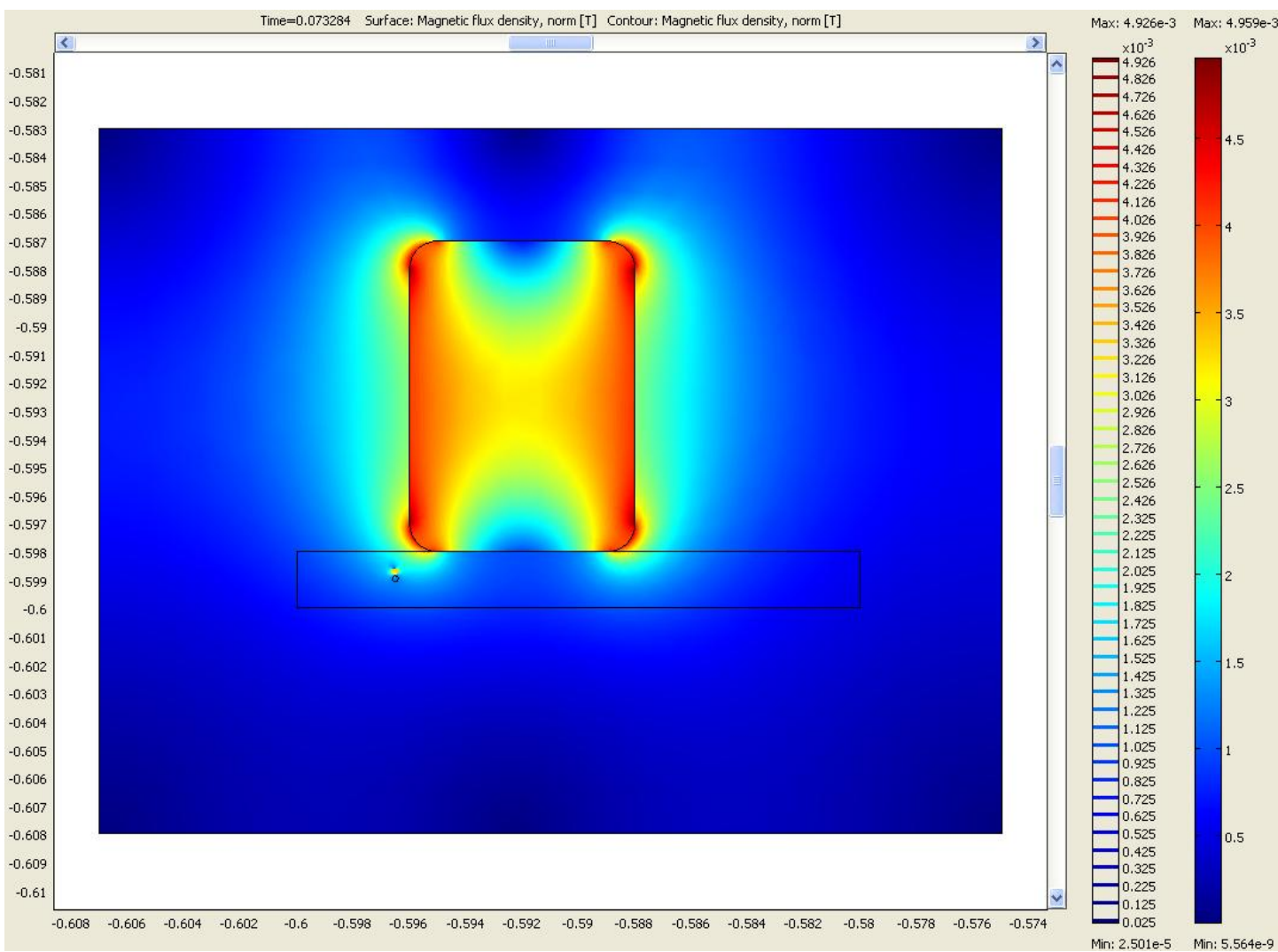

Figure 40: The Magnetic Field of the Particle Motion Model

Although this model was programmed to run for 10 seconds, inverted mesh elements caused the model to stop solving at 0.073284 seconds, illustrating slight movement of the particle toward the permanent magnet. Figure 41 depicts the movement of the particle for the model by plotting the fluid velocity on the surface of the channel and magnetic potential contour lines for the magnet. 


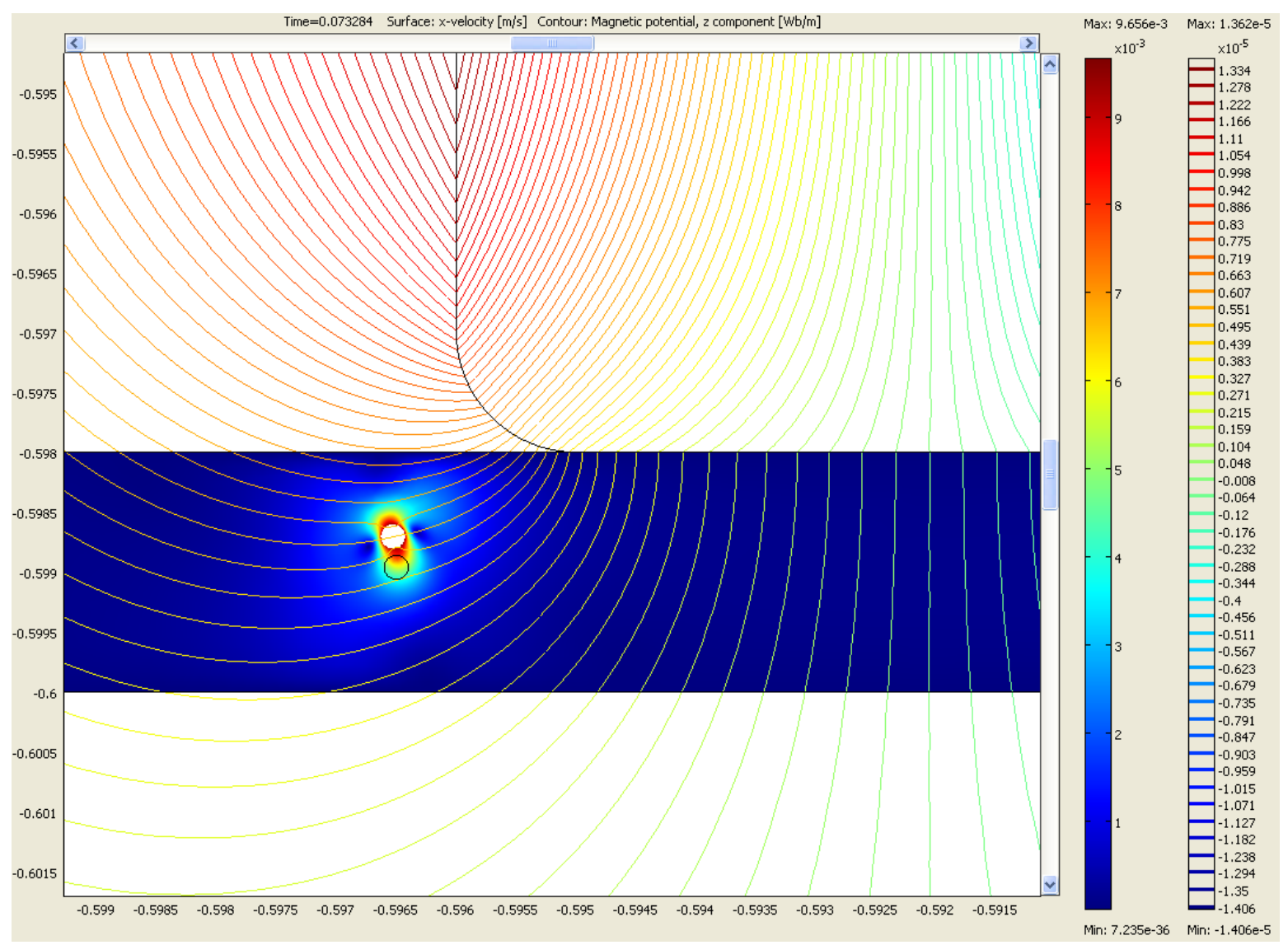

Figure 41: An Illustration of the Particle's Movement

The circle with the black line indicates the original location of the superparamagnetic particle while the white circle is its final location. Over the short solution period, the particle was able to move upward roughly $260 \mu \mathrm{m}$. As the velocity field indicates, the movement of this particle was the result of the attraction to the permanent magnet only. With a fluid velocity of $90 \mu \mathrm{m} / \mathrm{s}$ for the total solution time of $0.073284 \mathrm{~s}$, the fluid was only able to travel $6.6 \mu \mathrm{m}$ from the inlet of the channel. An illustration of the particle's movement starting with $0.01 \mathrm{~s}$ can be seen in Figure 42 through 45. 

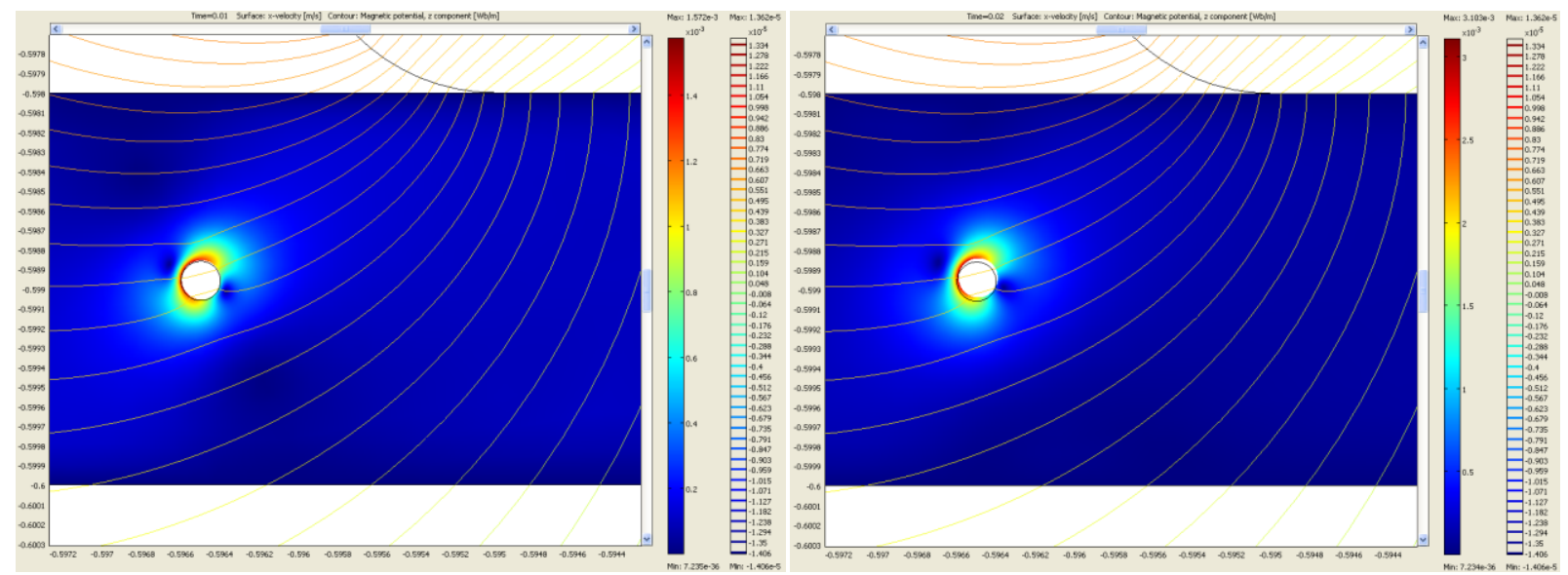

Figure 42: Particle Motion at $0.01 \mathrm{~s}$ (Left) and 0.02s (Right)
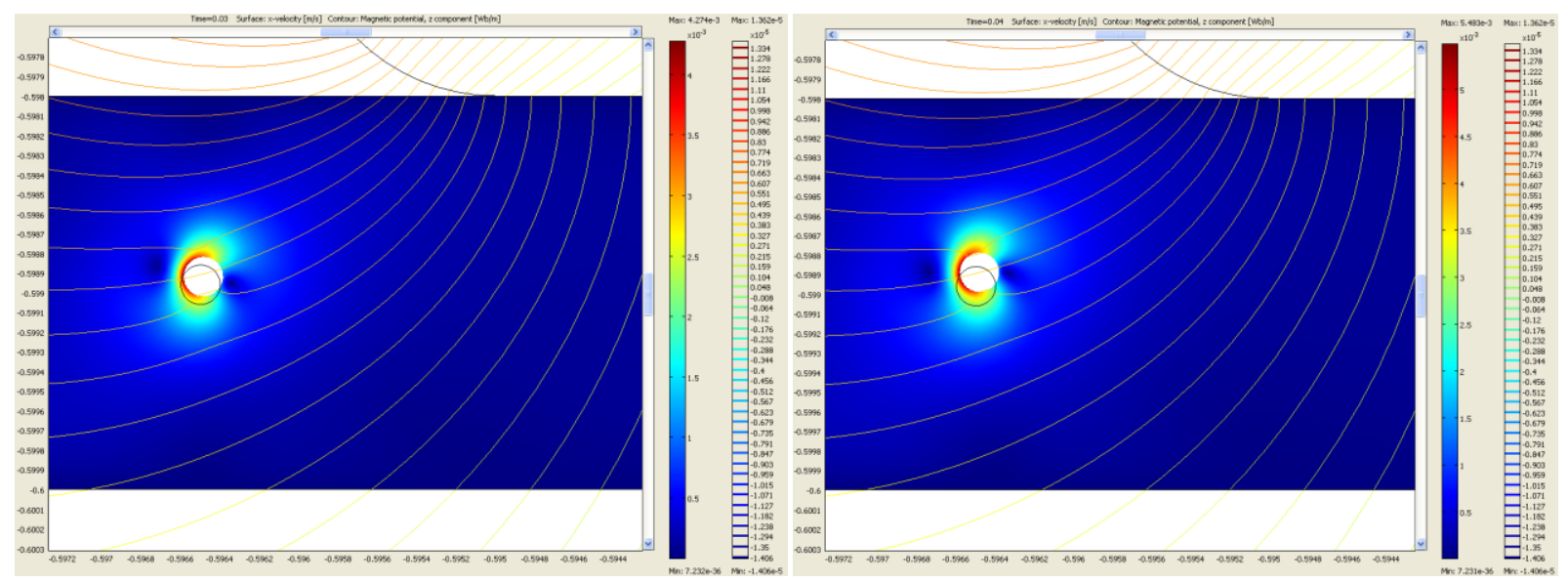

Figure 43: Particle Motion at $0.03 \mathrm{~s}$ (Left) and 0.04s (Right)

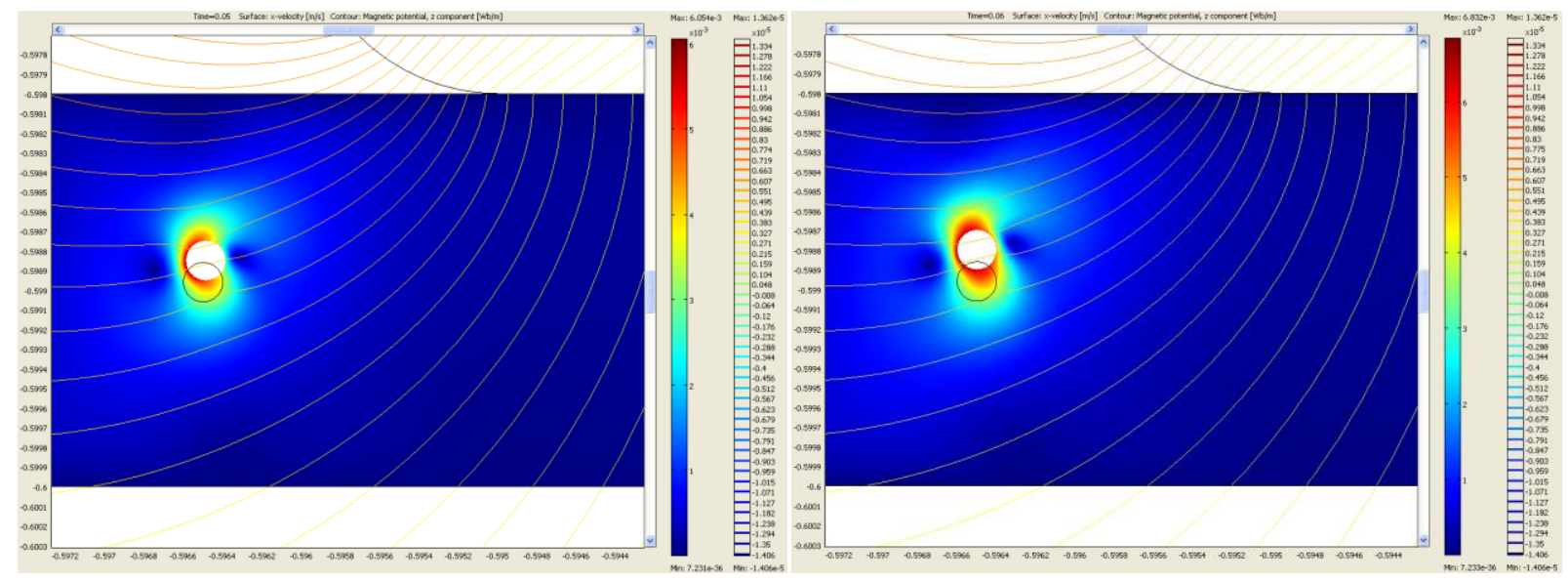

Figure 44: Particle Motion at $0.05 \mathrm{~s}$ (Left) and 0.06s (Right) 

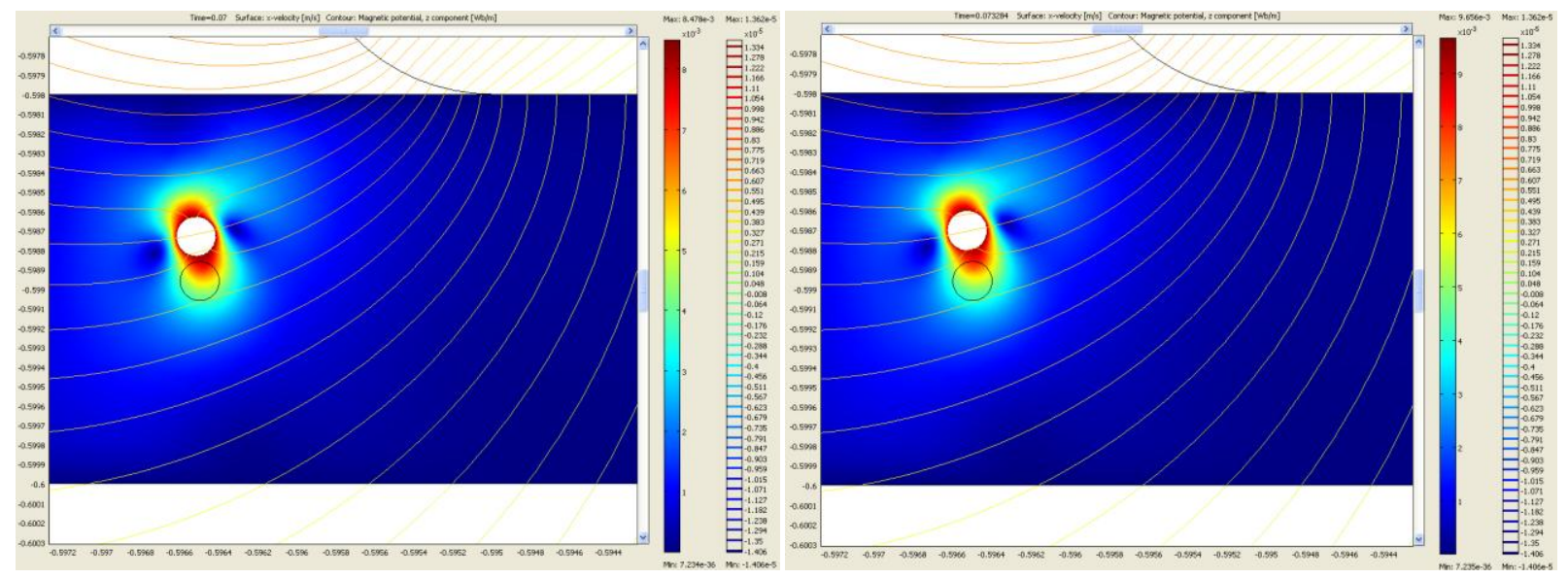

Figure 45: Particle Motion at $0.07 \mathrm{~s}$ (Left) and 0.073284s (Right)

Since the Particle Motion Model will not solve long enough for both fluid and magnetic forces to be taken into account in the movement of the particle, this model is insufficient for the desired applications of superparamagnetic particle modeling. As a result, no further research into the coupling of the Moving Mesh ALE, Incompressible Navier-Stokes, Plane Strain, and Magnetostatics modes was conducted.

\subsection{The Mixture Model}

The Mixture Model was successfully solved for all $10 \mathrm{~s}$; however, by that time, almost all of the superparamagnetic particle solution had left the channel. Consequently, Figure 46, which is a plot of volume fraction of the superparamagnetic particle solution in the channel and the magnetic potential contour lines, is the solution of the Mixture Model at $0.33 \mathrm{~s}$ when the superparamagnetic particle fluid is still passing beneath the permanent magnet. 


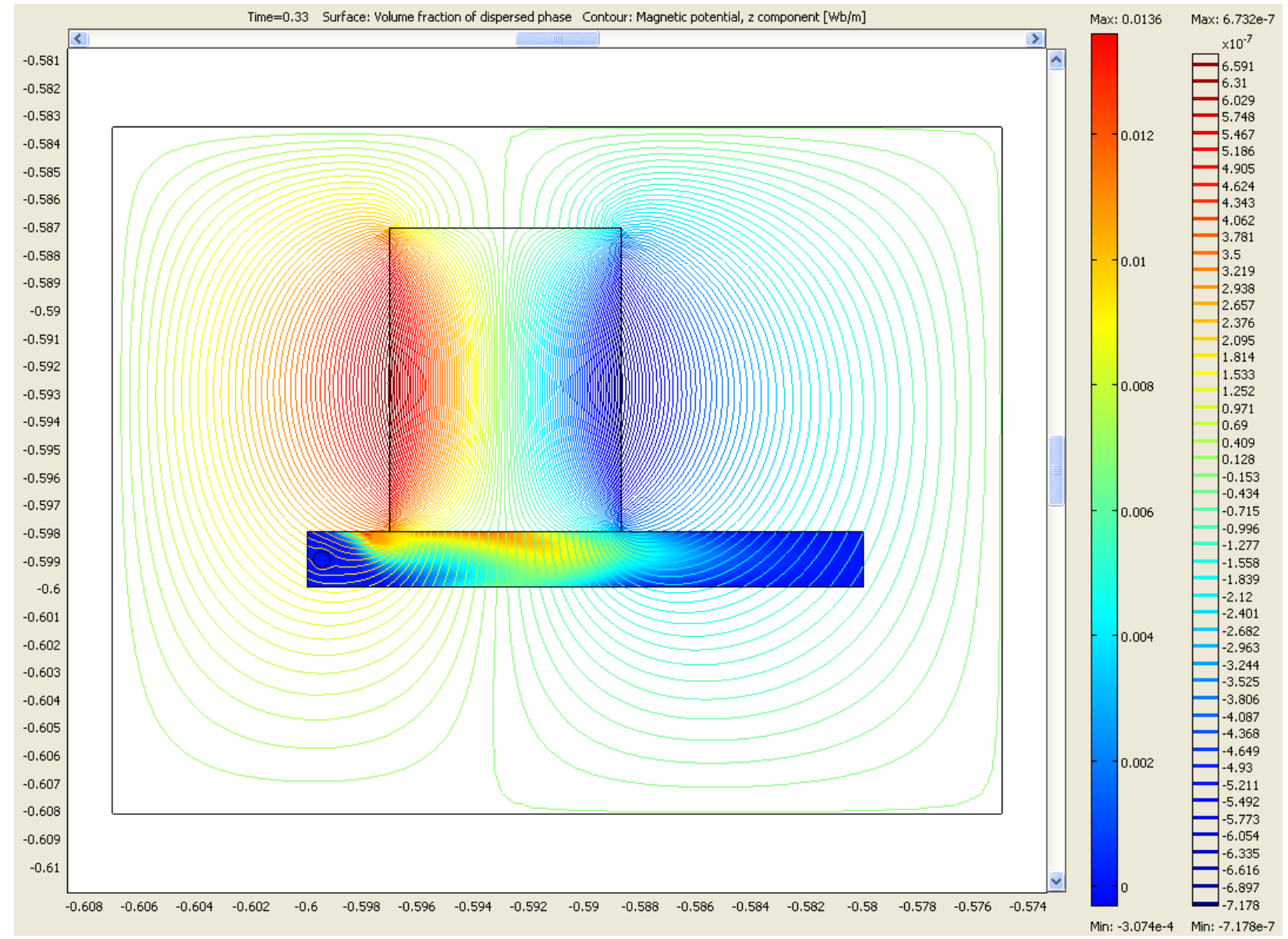

Figure 46: Surface Plot of the Superparamagnetic Fluid Volume Fraction and Magnetic Potential Contour Plot for the Mixture Model at $0.33 \mathrm{~s}$

With a magnetic potential similar to the one discussed in Section 5.1, the superparamagnetic particle fluid enters the fluidic channel through the circular inlet indicated by the black circle on the left side of the channel and is attracted to the permanent magnet. Figure 47 through Figure 55 illustrate the movement of the particle fluid for $0.85 \mathrm{~s}$ at $0.05 \mathrm{~s}$ time steps. 

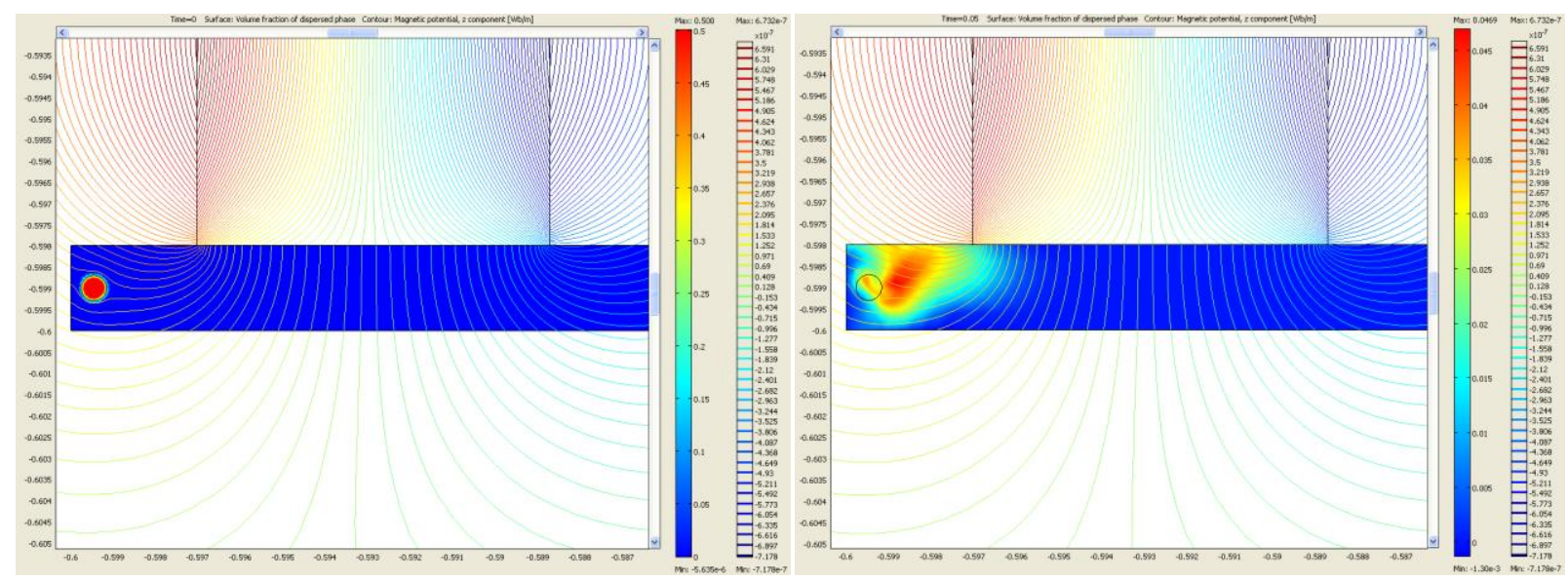

Figure 47: Superparamagnetic Particle Fluid Movement at $0 \mathrm{~s}$ (Left) and $0.05 \mathrm{~s}$ (Right) for the Mixture Model

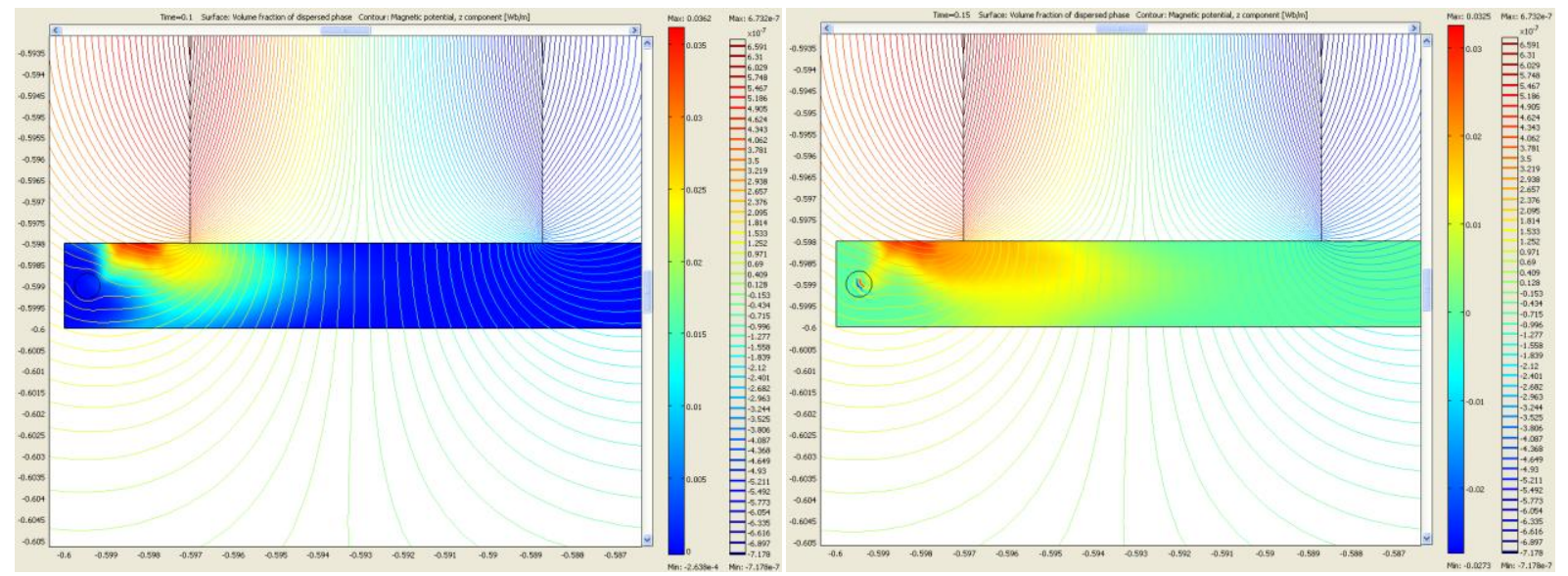

Figure 48: Superparamagnetic Particle Fluid Movement at $0.10 \mathrm{~s}$ (Left) and $0.15 \mathrm{~s}$ (Right) for the Mixture Model
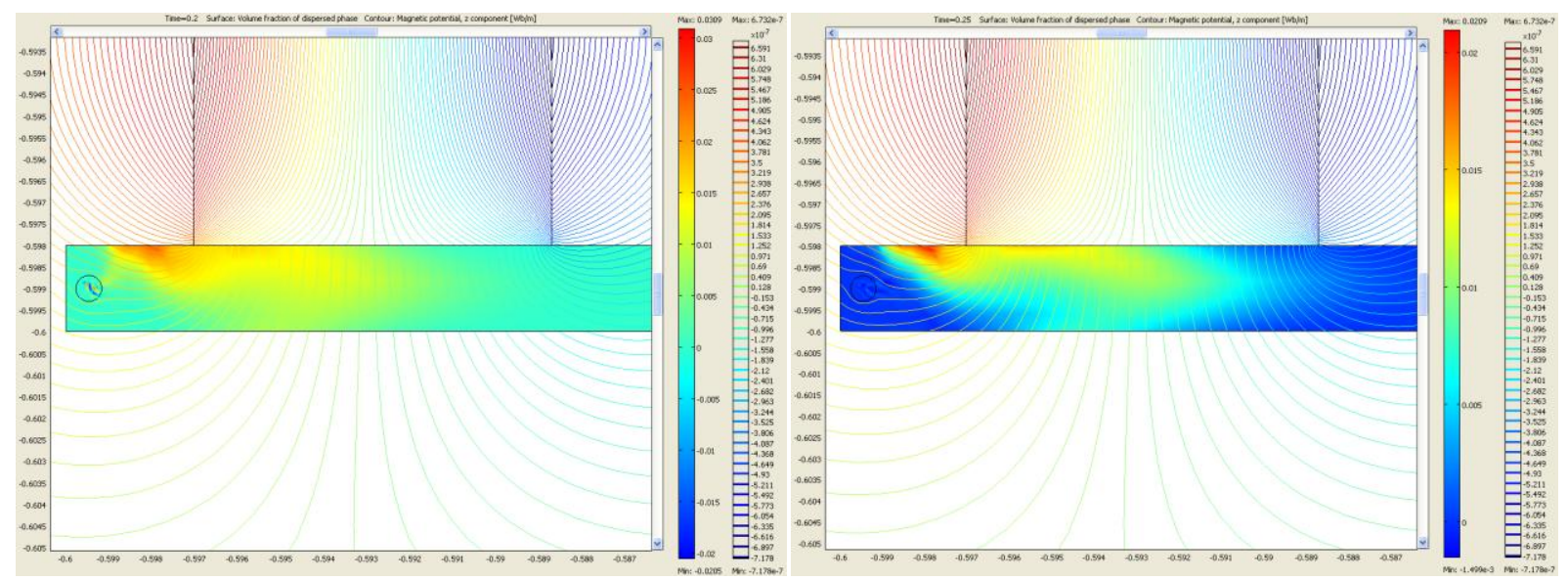

Figure 49: Superparamagnetic Particle Fluid Movement at $0.20 \mathrm{~s}$ (Left) and $0.25 \mathrm{~s}$ (Right) for the Mixture Model 

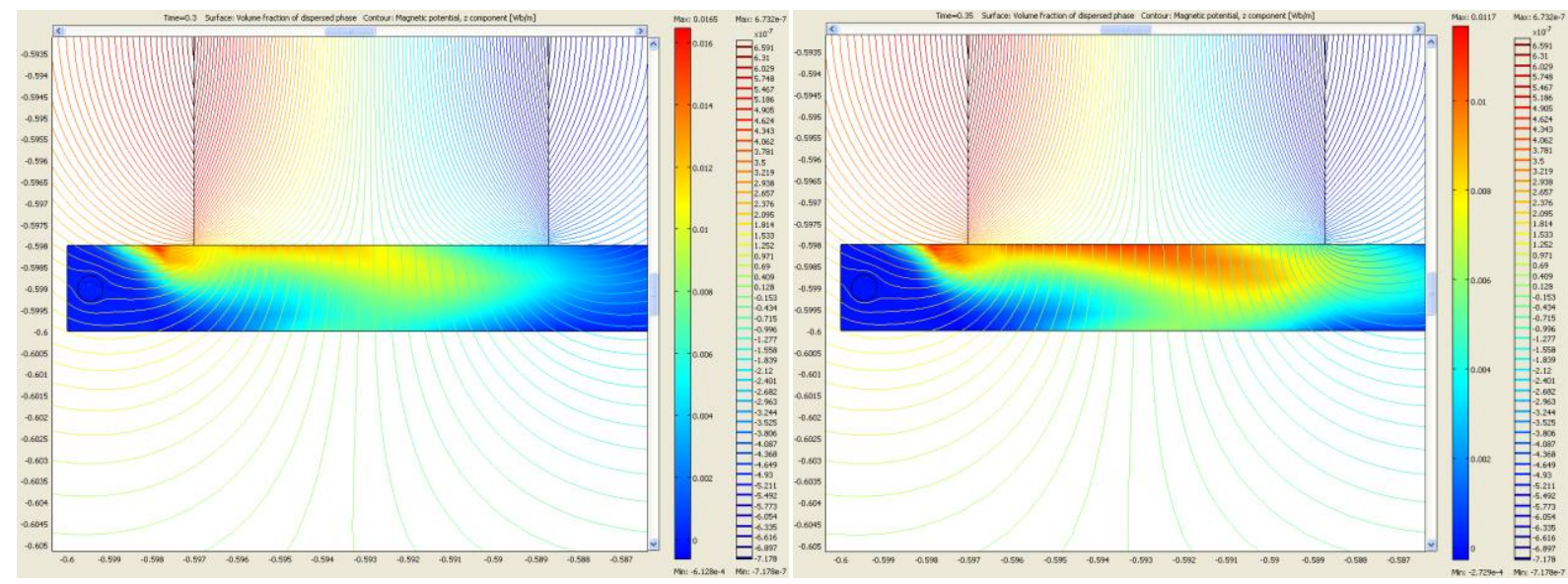

Figure 50: Superparamagnetic Particle Fluid Movement at $0.30 \mathrm{~s}$ (Left) and $0.35 \mathrm{~s}$ (Right) for the Mixture Model

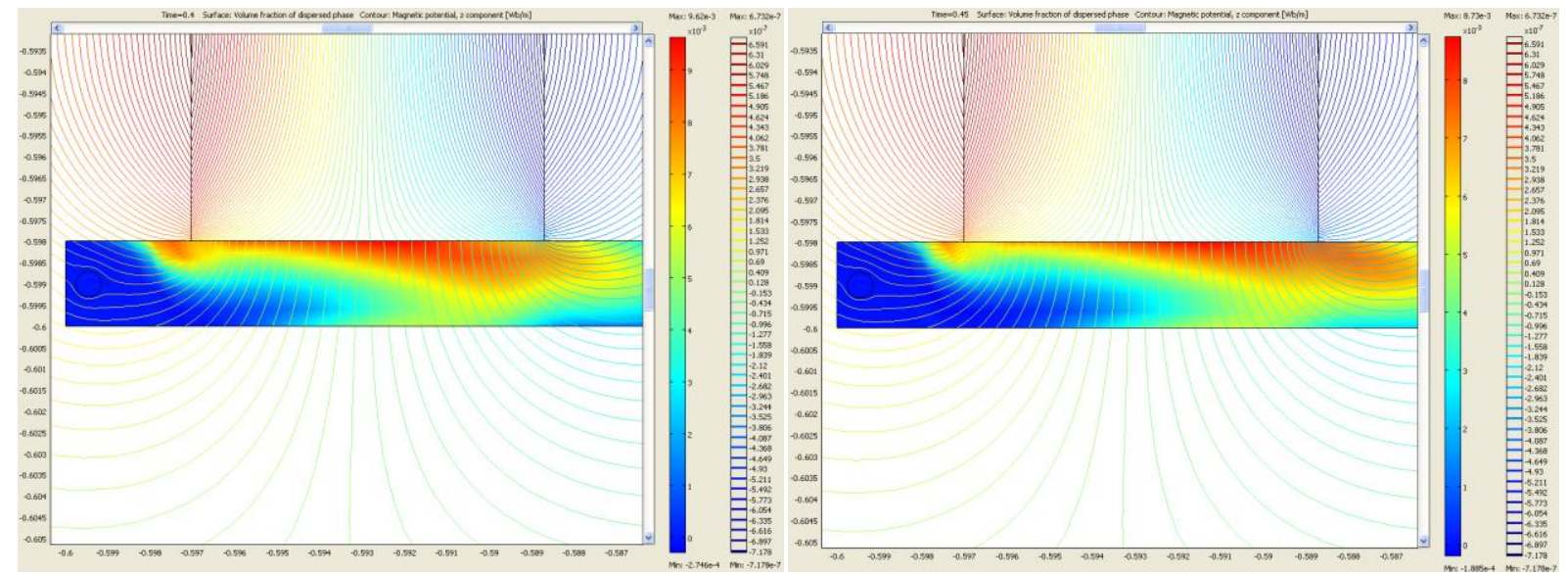

Figure 51: Superparamagnetic Particle Fluid Movement at $0.40 \mathrm{~s}$ (Left) and $0.45 \mathrm{~s}$ (Right) for the Mixture Model

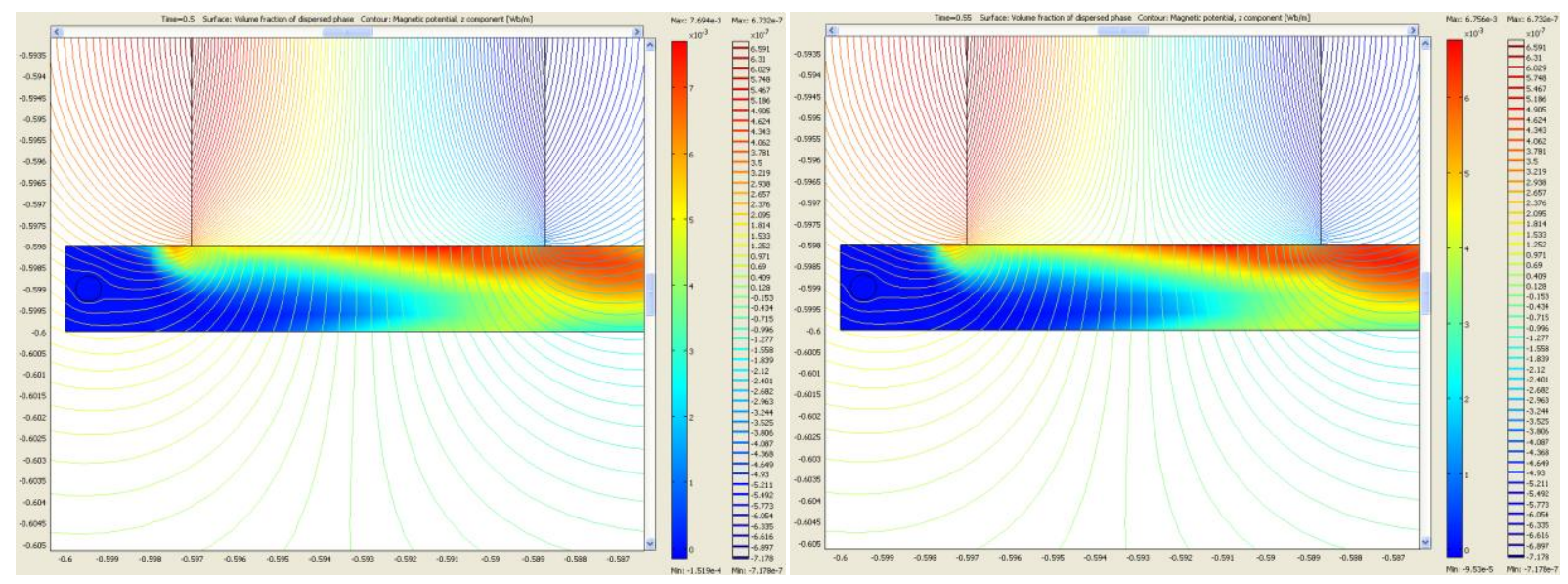

Figure 52: Superparamagnetic Particle Fluid Movement at $0.50 \mathrm{~s}$ (Left) and $0.55 \mathrm{~s}$ (Right) for the Mixture Model 


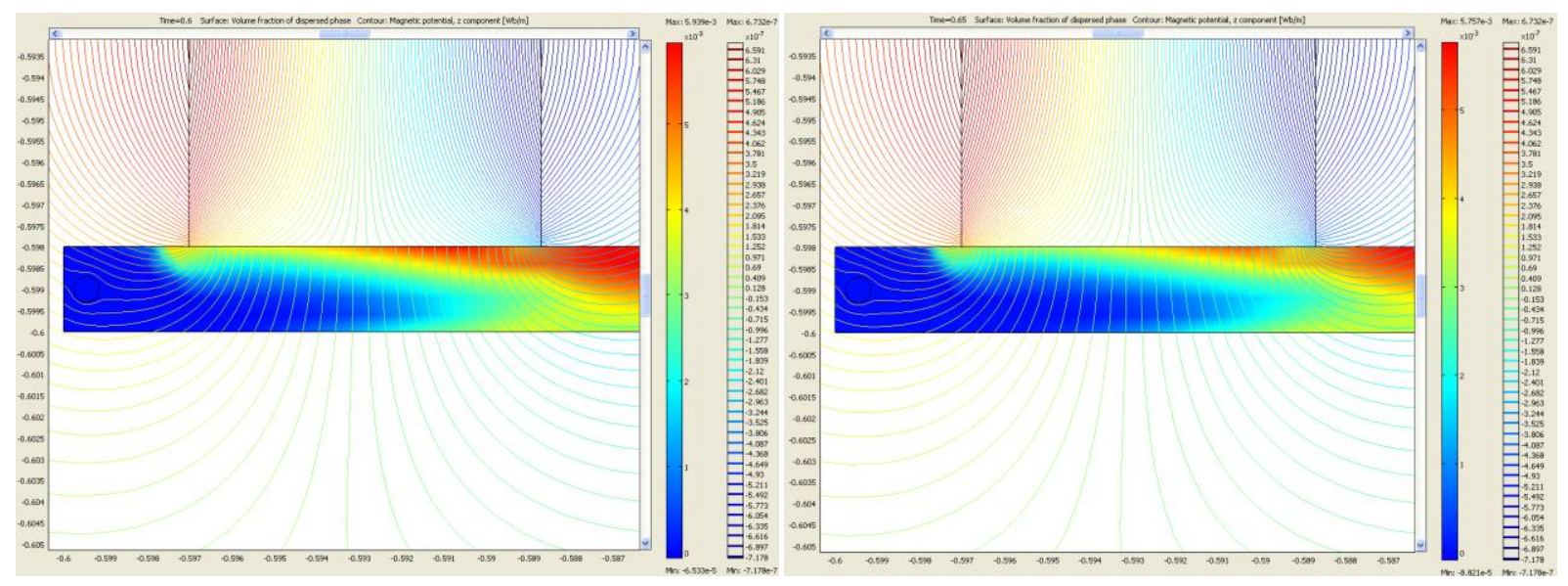

Figure 53: Superparamagnetic Particle Fluid Movement at $0.60 \mathrm{~s}$ (Left) and $0.65 \mathrm{~s}$ (Right) for the Mixture Model

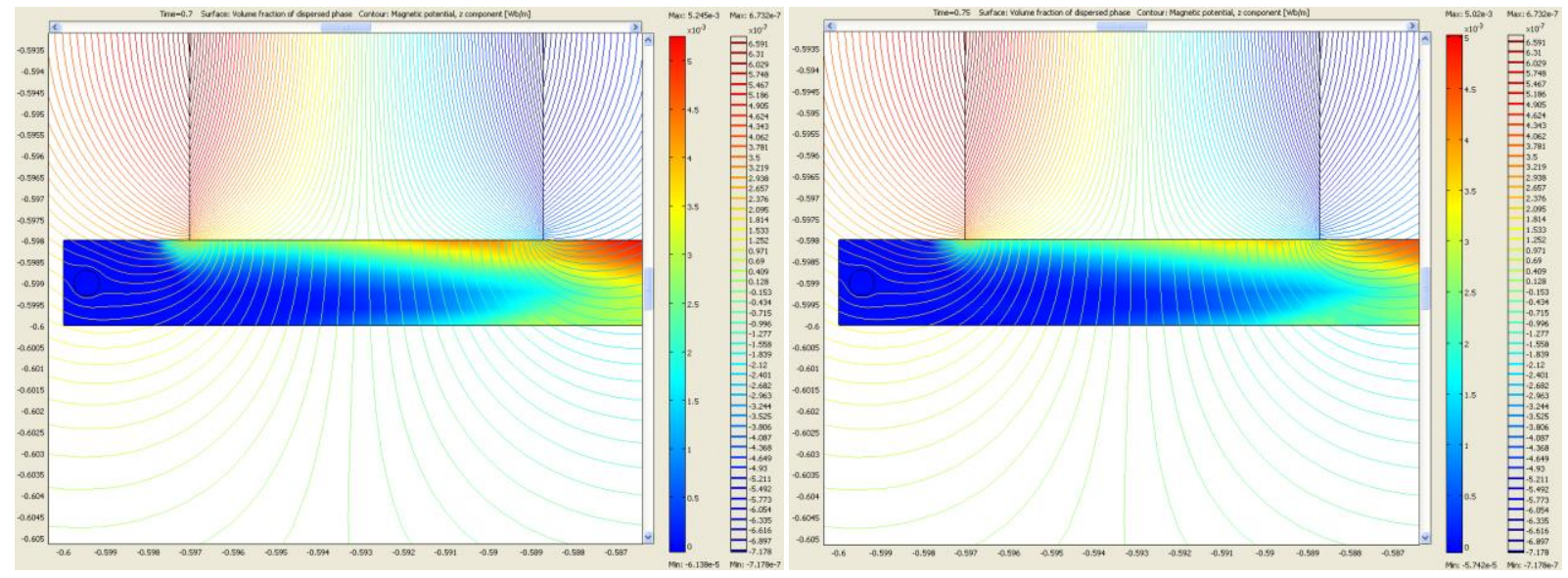

Figure 54: Superparamagnetic Particle Fluid Movement at $0.70 \mathrm{~s}$ (Left) and $0.75 \mathrm{~s}$ (Right) for the Mixture Model

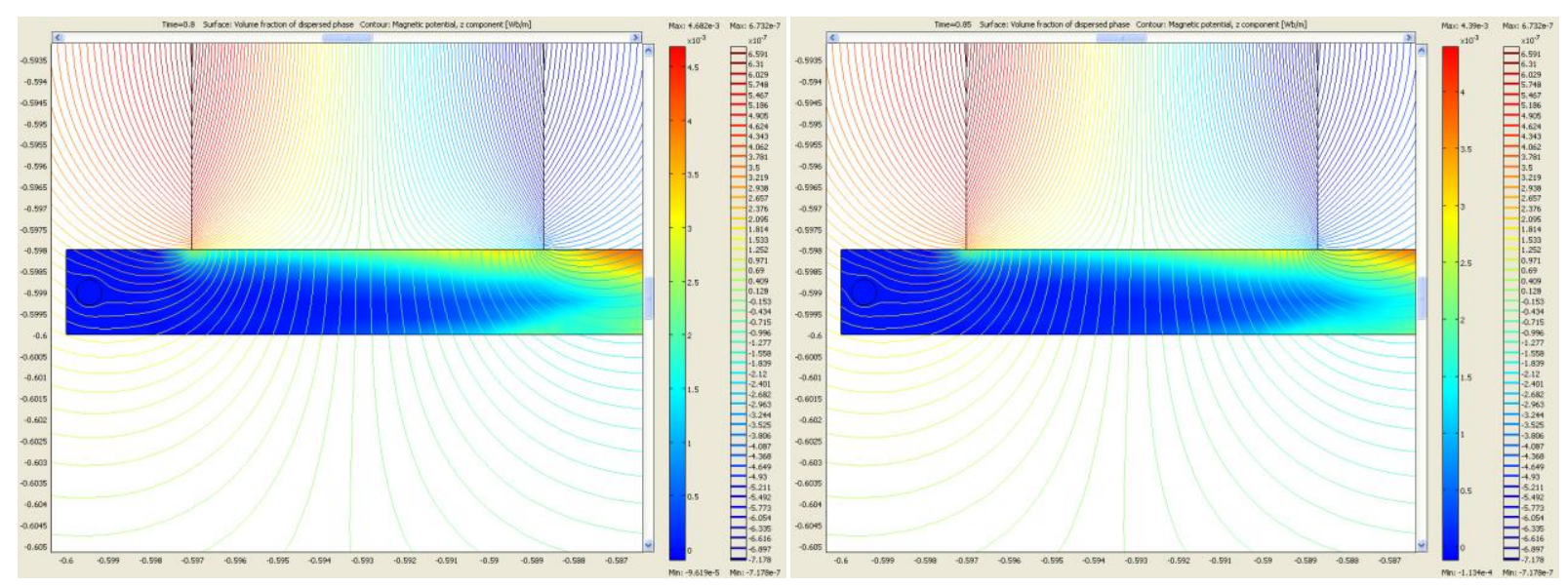

Figure 55: Superparamagnetic Particle Fluid Movement at $0.80 \mathrm{~s}$ (Left) and $0.85 \mathrm{~s}$ (Right) for the Mixture Model 
The dark red coloring indicates the highest concentration of the superparamagnetic particles; however, for the above images, the legends are not the same for the various times. As a result, volume fraction plots for all of the solution times can be created for various locations in the channel. Figure 56 illustrates the volume fraction versus vertical channel location given in "Arc-length" beneath the left edge of the permanent magnet. The different lines of the plot indicate the different solution times of the model for the largest volume fractions at the specified location beneath or above the permanent magnet. The "Arc-length" is the vertical distance from the top of the channel, which is closest to the magnet in the current orientation, for the total distance of the entire height of the channel or $2 \mathrm{~mm}$. For quantifying the success of particle attraction within the channel, the middle of the channel is identified as the $1 \mathrm{e}-3$ $m$ length on the plot and the volume fraction for the solution times are compared on either side of this line. If the particles were not captured or attracted to the permanent magnet, the volume fraction lines would be vertical for the entire height of the channel. A visual sway towards the top or bottom of the channel would indicate attraction or repulsion, respectively.

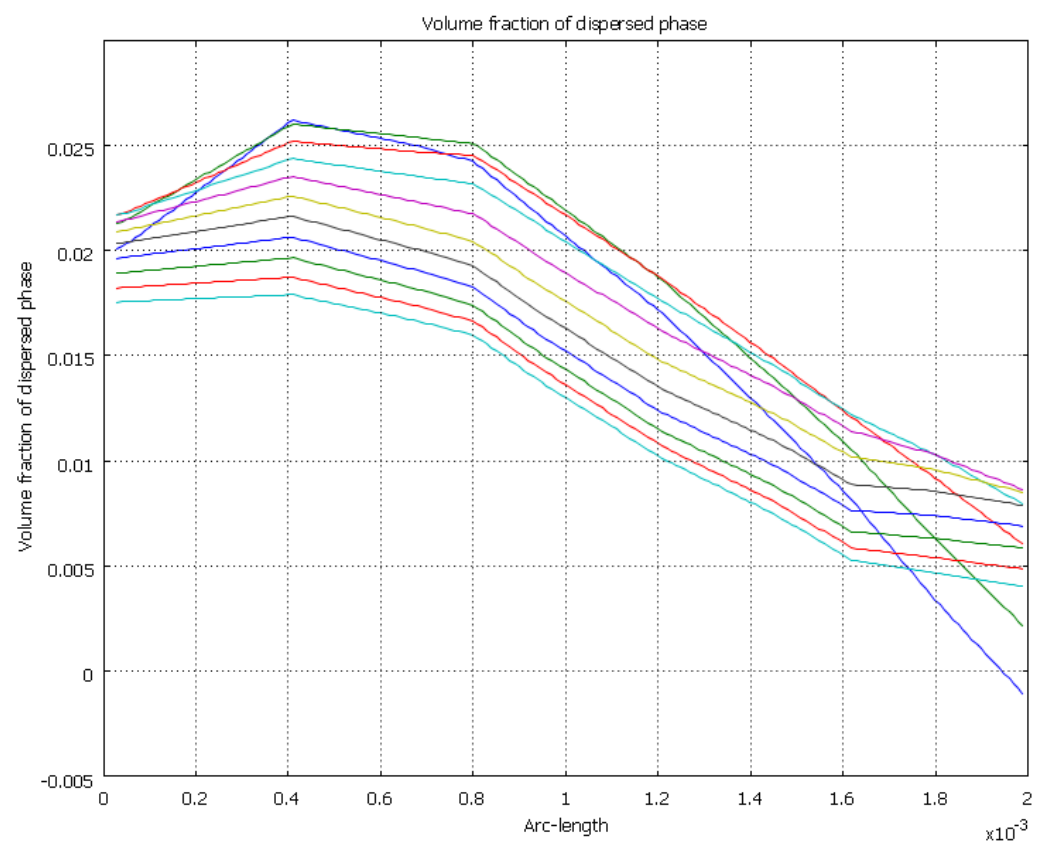

Figure 56: Volume Fraction of the Superparamagnetic Particle Fluid beneath the Left Side of the Magnet 
Figure 56 indicates that the superparamagnetic particle solution is highly attracted to the permanent magnet with the volume fraction beneath this location decreasing considerably over the height of the channel from a maximum volume fraction of roughly 0.026 to 0 for a few of the earliest solution times.

Beneath the middle of the magnet where the magnetic potential is the smallest, the particle fluid is less attracted to the permanent magnet as seen in Figure 57. However, compared to the edge of the magnet, the difference in the volume fraction over the height of the channel is not as large, indicating that the superparamagnetic particles are not as attracted to the magnet beneath the middle of the magnet.

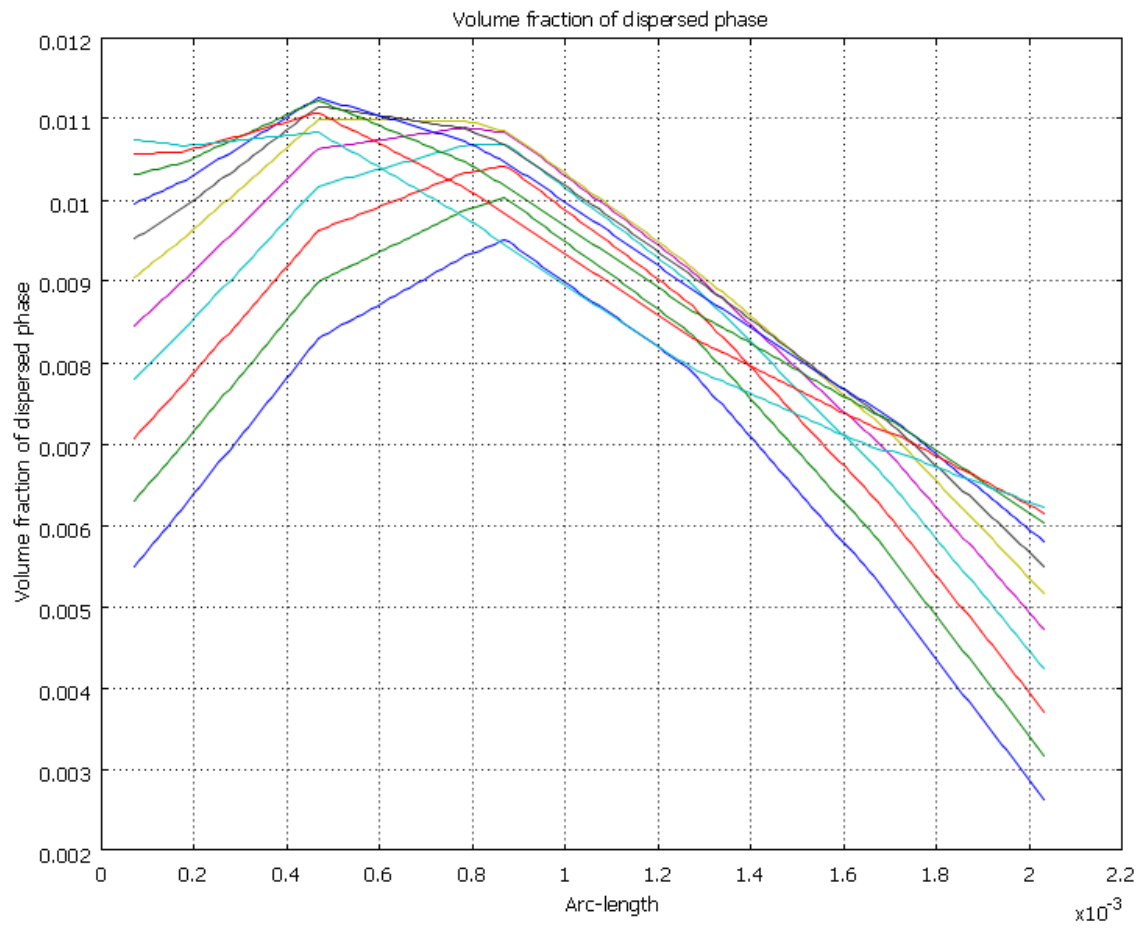

Figure 57: Volume Fraction of the Superparamagnetic Particle Fluid beneath the Middle of the Magnet

Between the edge of the magnet and the middle of the magnet, roughly $52 \%$ of the maximum volume fraction has been lost as a result of diffusion and the reduced magnetic field. The volume fraction is reduced even further by the time the superparamagnetic particles reach the right edge of the magnet as seen in Figure 58. 


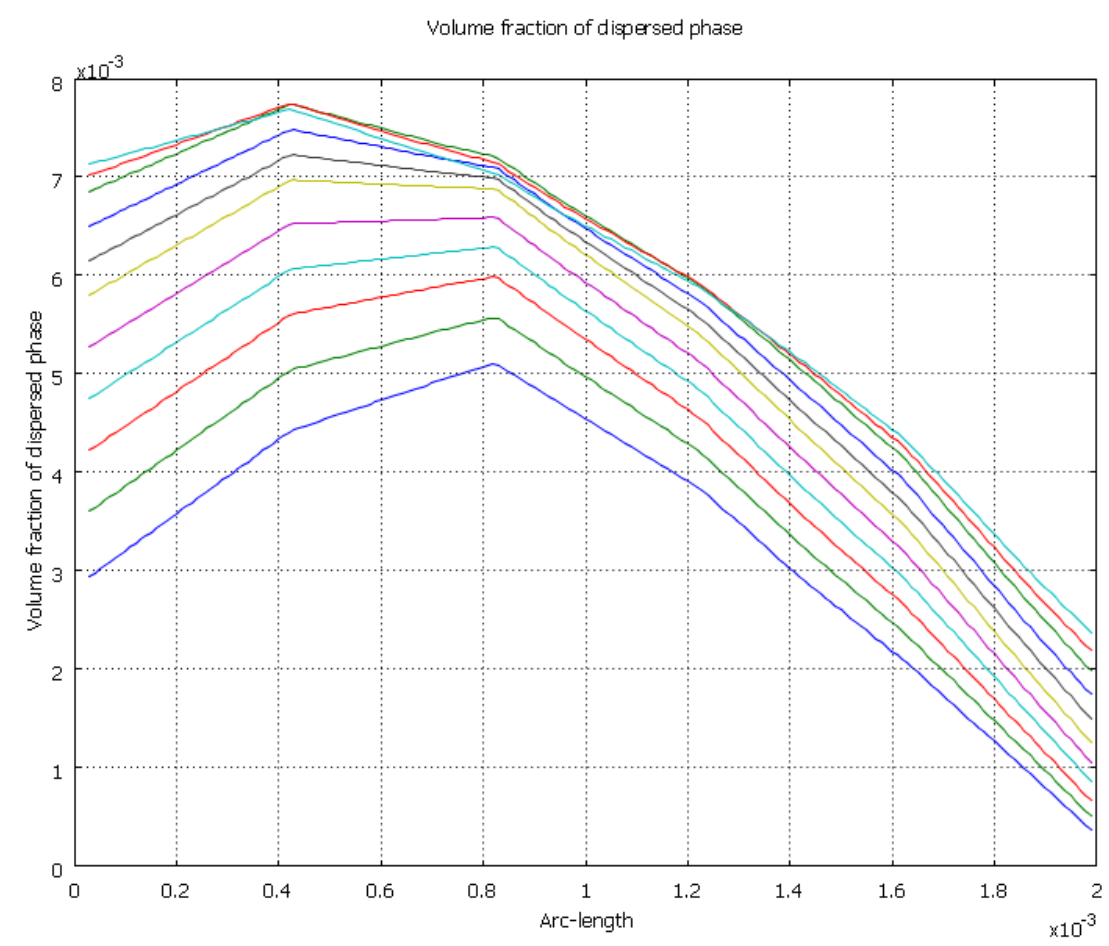

Figure 58: Volume Fraction of the Superparamagnetic Particle Fluid beneath the Right Side of the Magnet

As with the previous reduction, the much smaller magnetic field exerted on the particles beneath the middle of the permanent magnet results in the hydrodynamic forces from the fluid overcoming the magnetic forces and causing the particles to disperse away from the magnet. By the time the particles reach the stronger magnetic field on the right side of the magnet, the numerous particles have traveled away from the magnet and are unable to recover.

\subsubsection{Design Optimization Using the Mixture Model}

Using the volume fraction capabilities of the Mixture Model, design optimization of the system described in Sections 1.6 and 1.7 can be attempted by changing various parameters including velocity, geometry size and orientation, and magnetic field strength prior to experimentation. For the parameters described in Section 4.2, inertial effects were neglected for simplification. Since settling will occur experimentally, inertial effects should be included for design optimization. The inlet pressure and 
geometry size will remain the same as a hypothetical limitation of the fluid pump and manufacturing capabilities available for the system. To include the inertial effects of the system, the value for gravity, $9.81 \mathrm{~m} / \mathrm{s}^{2}$, is included in the $y$-component for "Gravity" in the "Physics" Subdomain Settings of the Mixture mode. Including gravity in the model results in a slight change on the system as seen in Figure 59 compared to Figure 46.
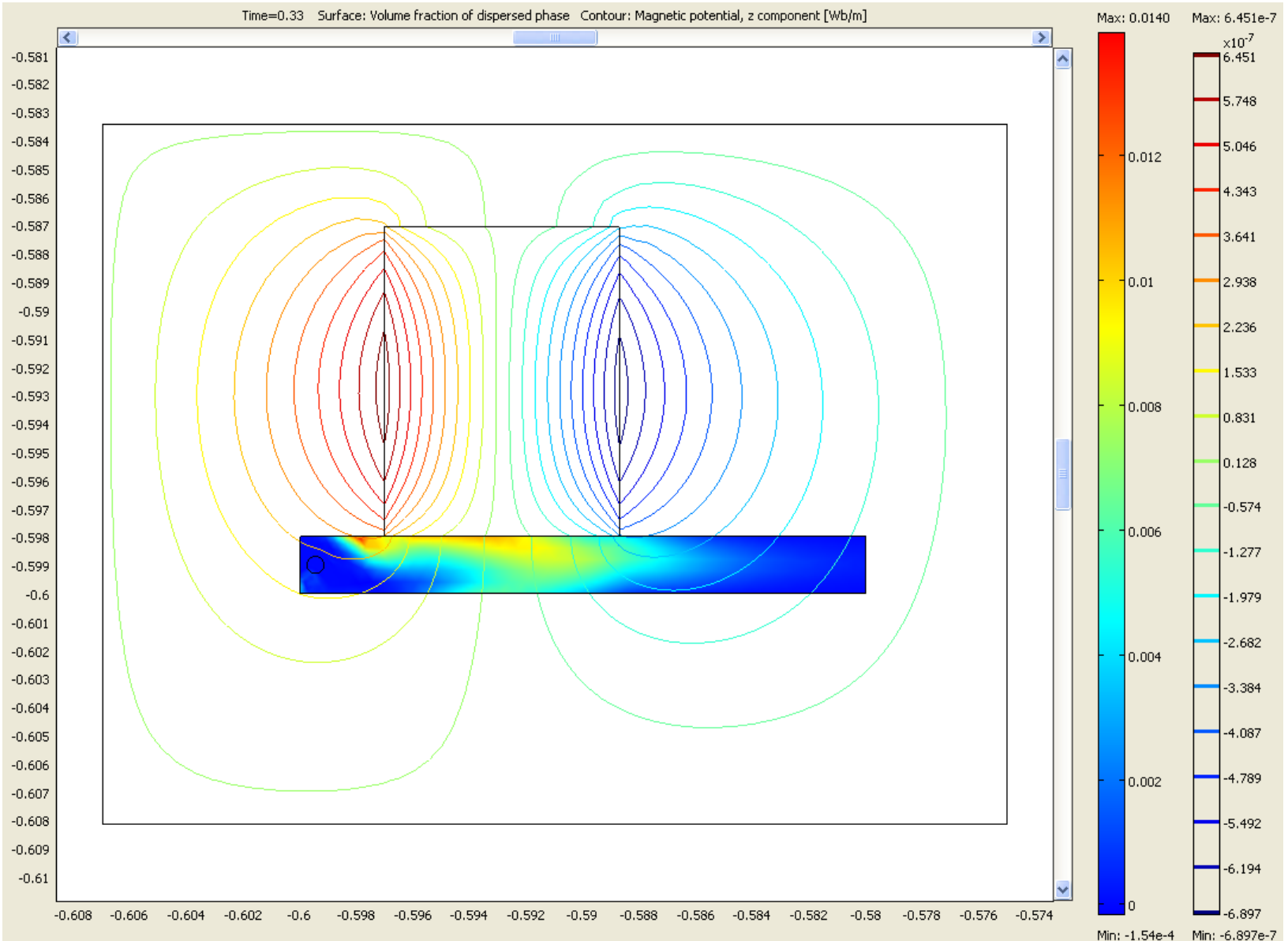

Figure 59: The Volume Fraction Surface Plot and Magnetic Potential Contour Plot for a Mixture Model that Includes Inertial Effects

The inertial effects can be further visualized by plotting the volume fraction of the superparamagnetic particles. These plots for beneath the left side, middle, and right side of the magnet can be seen in Figure 57 through 62. 


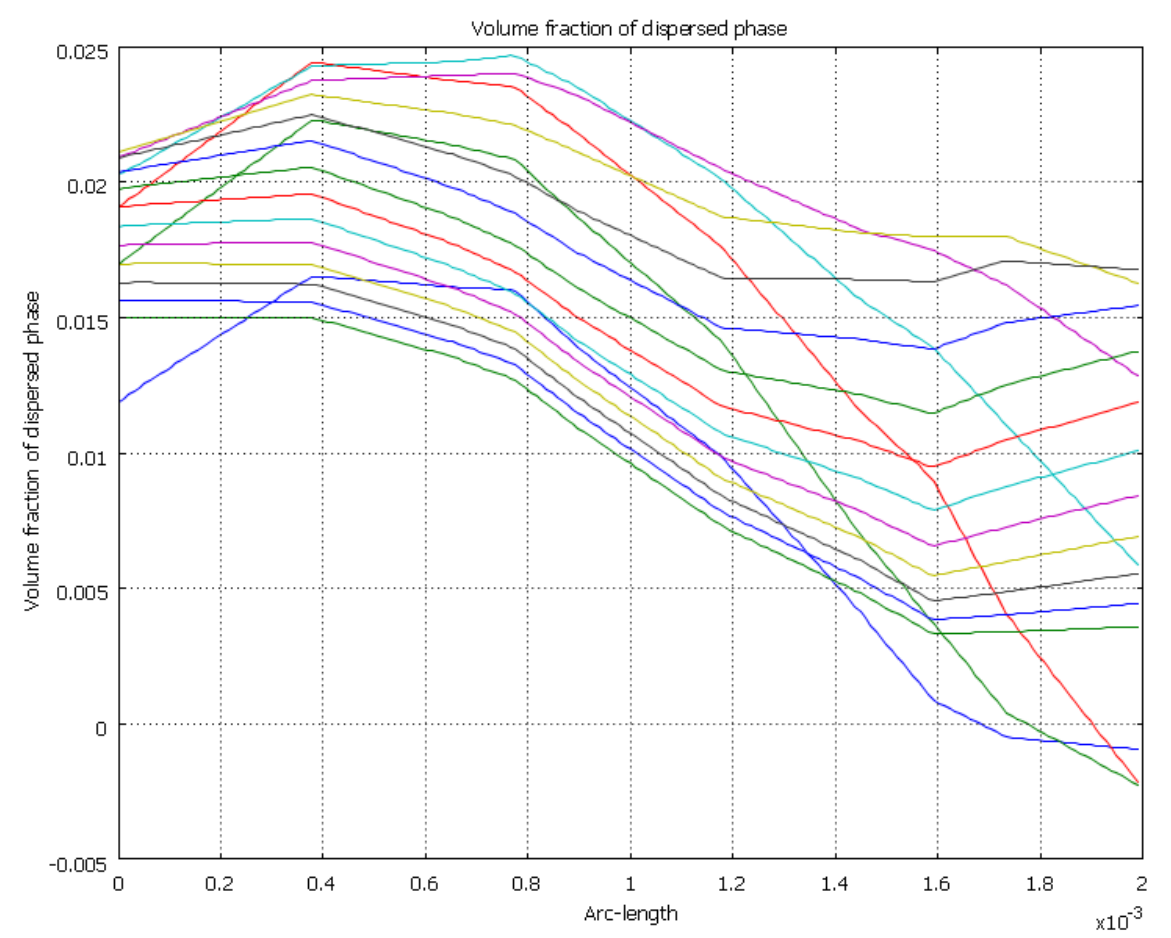

Figure 60: The Volume Fraction Plot for the Inertial Mixture Model beneath the Left Side of the Magnet

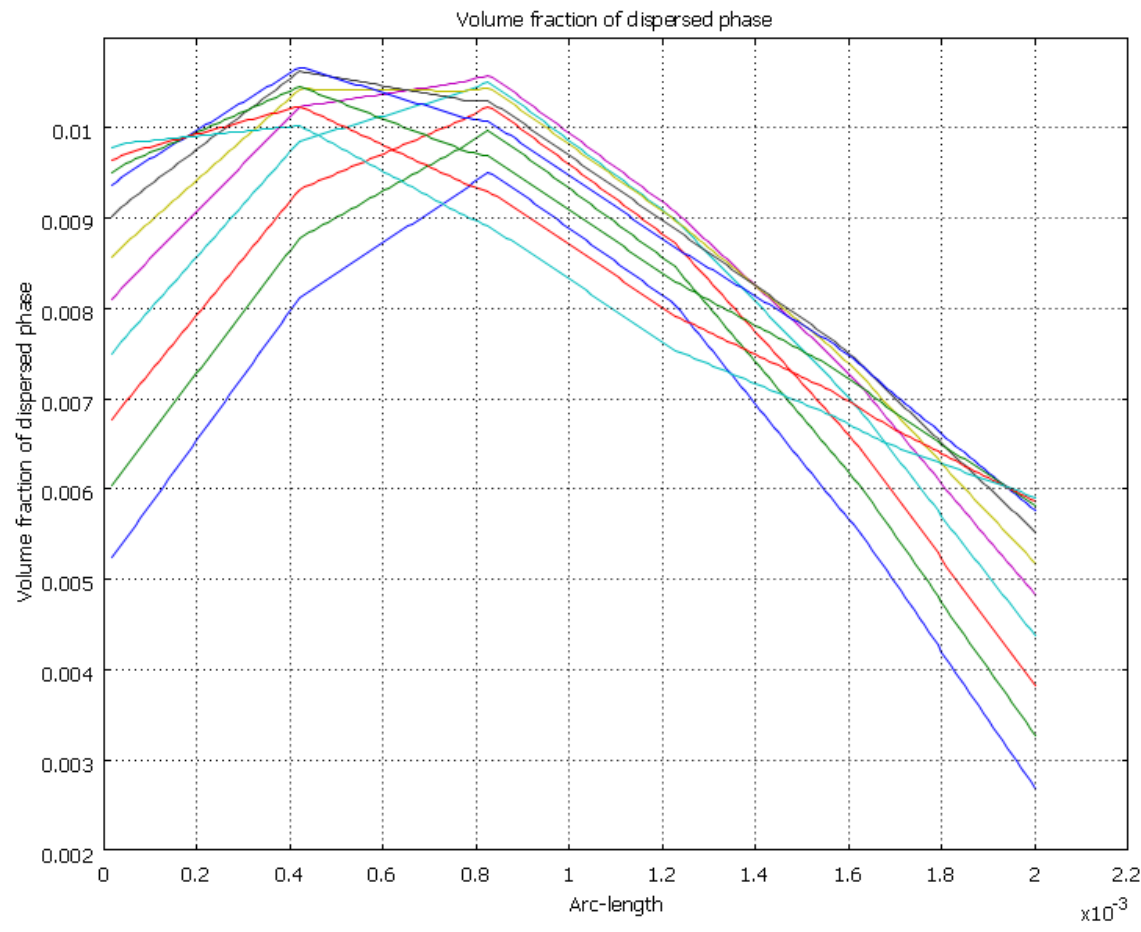

Figure 61: The Volume Fraction Plot for the Inertial Mixture Model beneath the Middle of the Magnet 


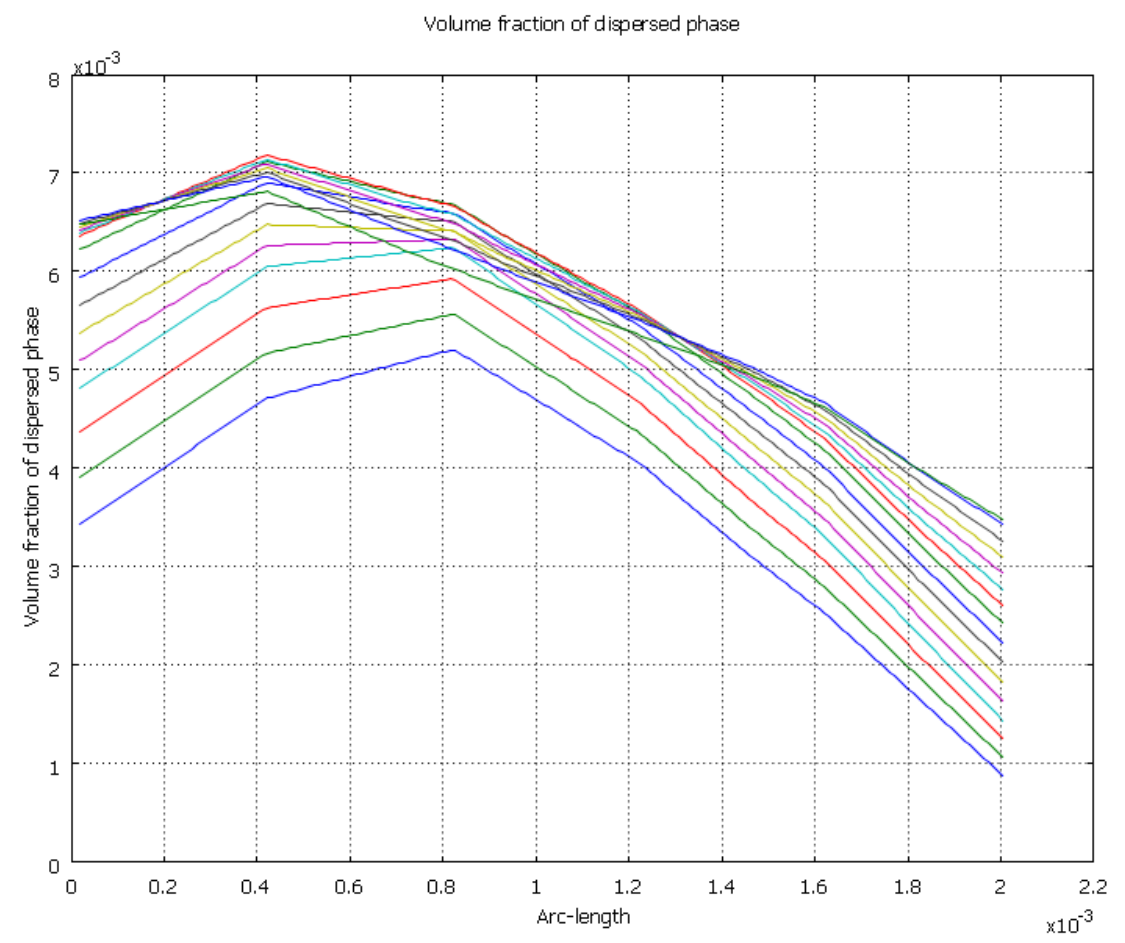

Figure 62: The Volume Fraction Plot for the Inertial Mixture Model beneath the Right Side of the Magnet

Compared to Figure 56 through Figure 58, gravity reduces the volume fraction closest to the magnet in all locations slightly as seen in Figure 60 through 62 . Since gravity has a downward effect on the superparamagnetic particle, optimization of the model would entail switching the location of the channel and magnet so that the channel rests upon the magnet. With this orientation, both the magnetic and inertial effects will act in the same direction. Figure 63 illustrates the $\mathrm{COMSOL}^{\circledR}$ solution for this orientation at $0.33 \mathrm{~s}$. 


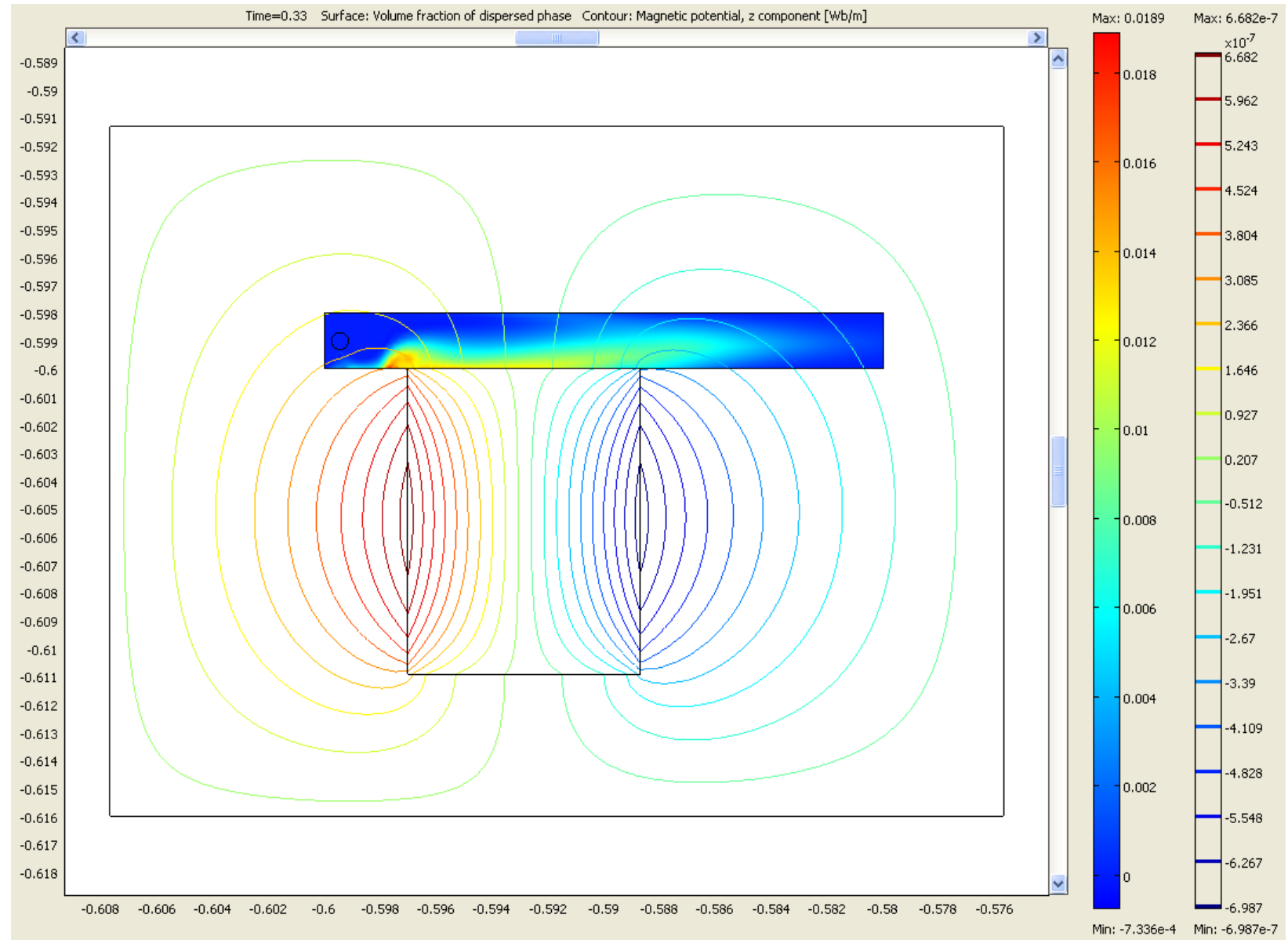

Figure 63: The Volume Fraction Surface Plot and Magnetic Potential Contour Plot of a Mixture Model with Reverse Orientation of the Channel and Magnet

To determine if the model alteration is an improvement from the initial orientation, the volume fraction of the superparamagnetic particles is again plotted beneath the left side, middle, and right side of the permanent magnet in Figures 64 through 66. 


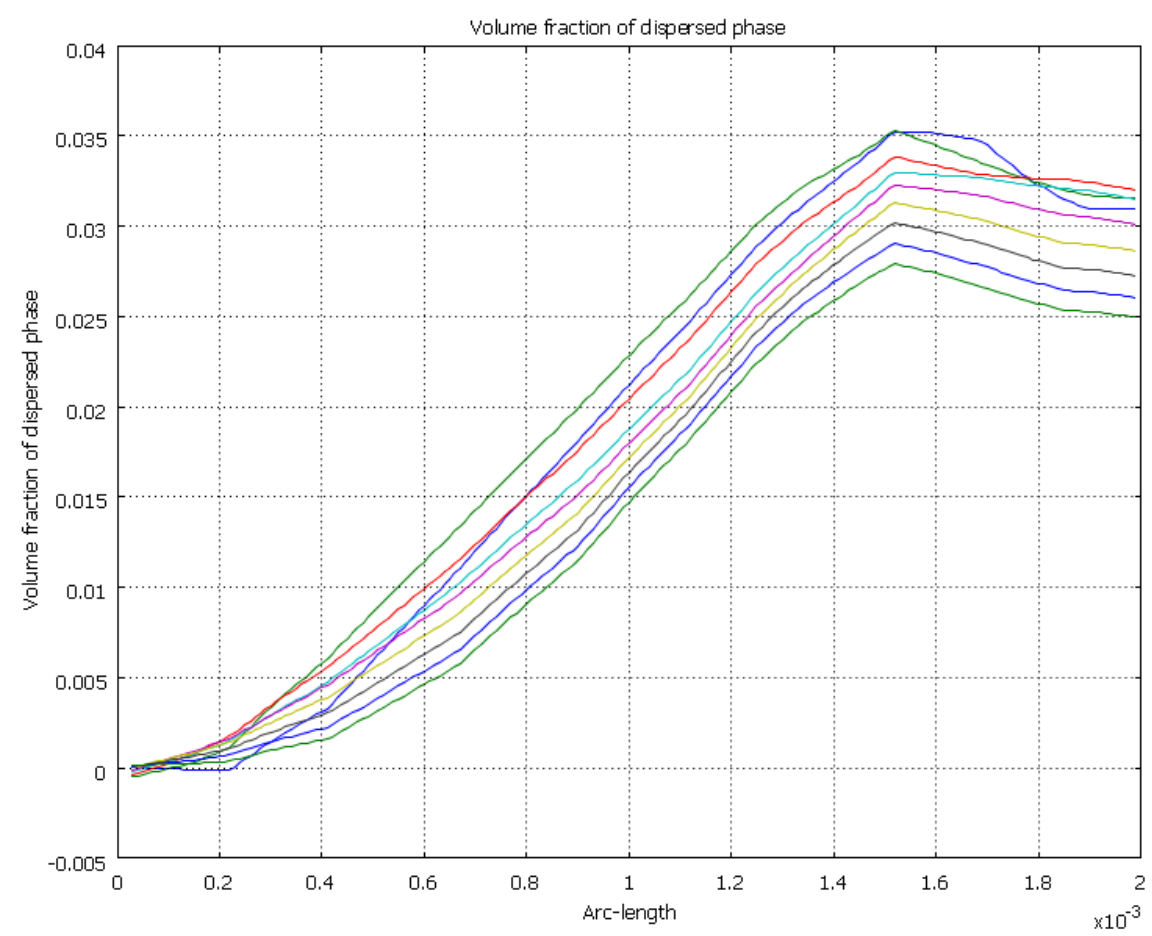

Figure 64: The Volume Fraction Plot for the Reversed Orientation Mixture Model above the Left Side of the Magnet

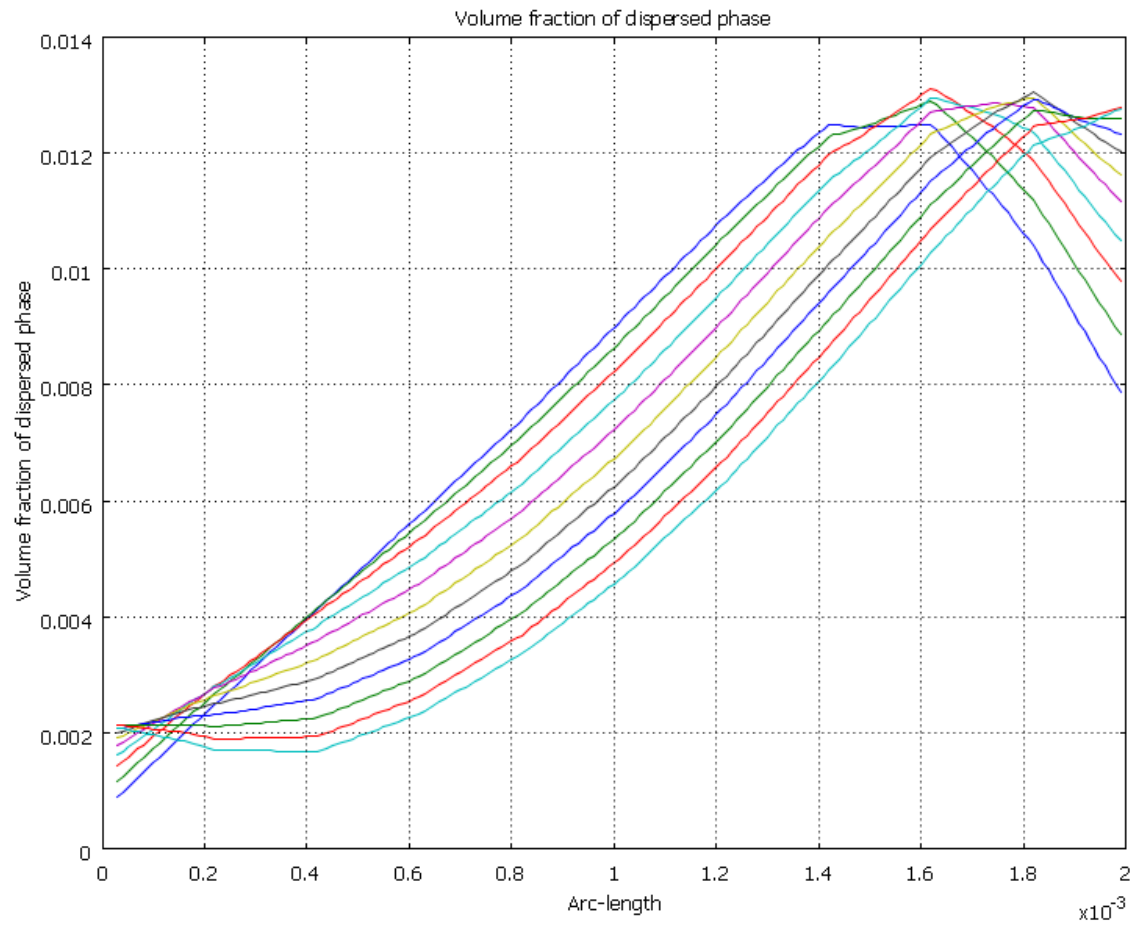

Figure 65: The Volume Fraction Plot for the Reversed Orientation Mixture Model above the Middle of the Magnet 


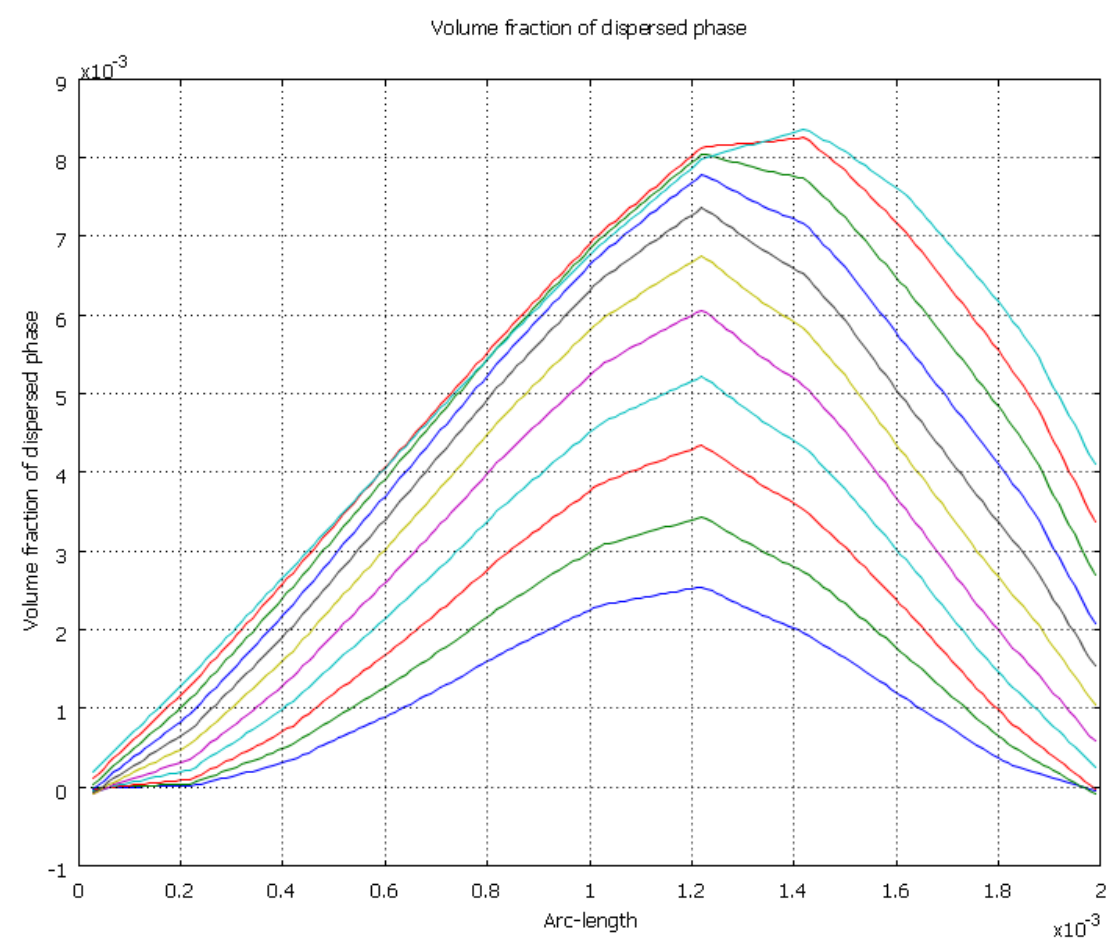

Figure 66: The Volume Fraction Plot for the Reversed Orientation Mixture Model above the Right Side of the Magnet

Since the orientation of the channel and magnet has been reversed, the left side of the volume fraction plots represents the channel height furthest from the permanent magnet. As a result, a sway towards the left indicates repulsion, and a sway towards the right indicates attraction to the magnet. Compared to Figures 56 through 58 and Figures 60 through 61, the maximum volume fraction has increased by approximately $0.01,0.001$, and 0.004 beneath the left side, middle, and right side of the magnet, respectively. In addition, the difference in volume fraction present between the bottom and top of the magnet is greatly increased, indicating greater system attraction.

For determining an optimum magnetic field strength for the trapping system, the remnant flux density of the magnet can be increased and decreased under the Magnetostatics Subdomains Settings. In the first change, the magnetic field strength is increased to $1 \mathrm{~T}$, producing the superparamagnetic fluid movement seen in Figure 67. 


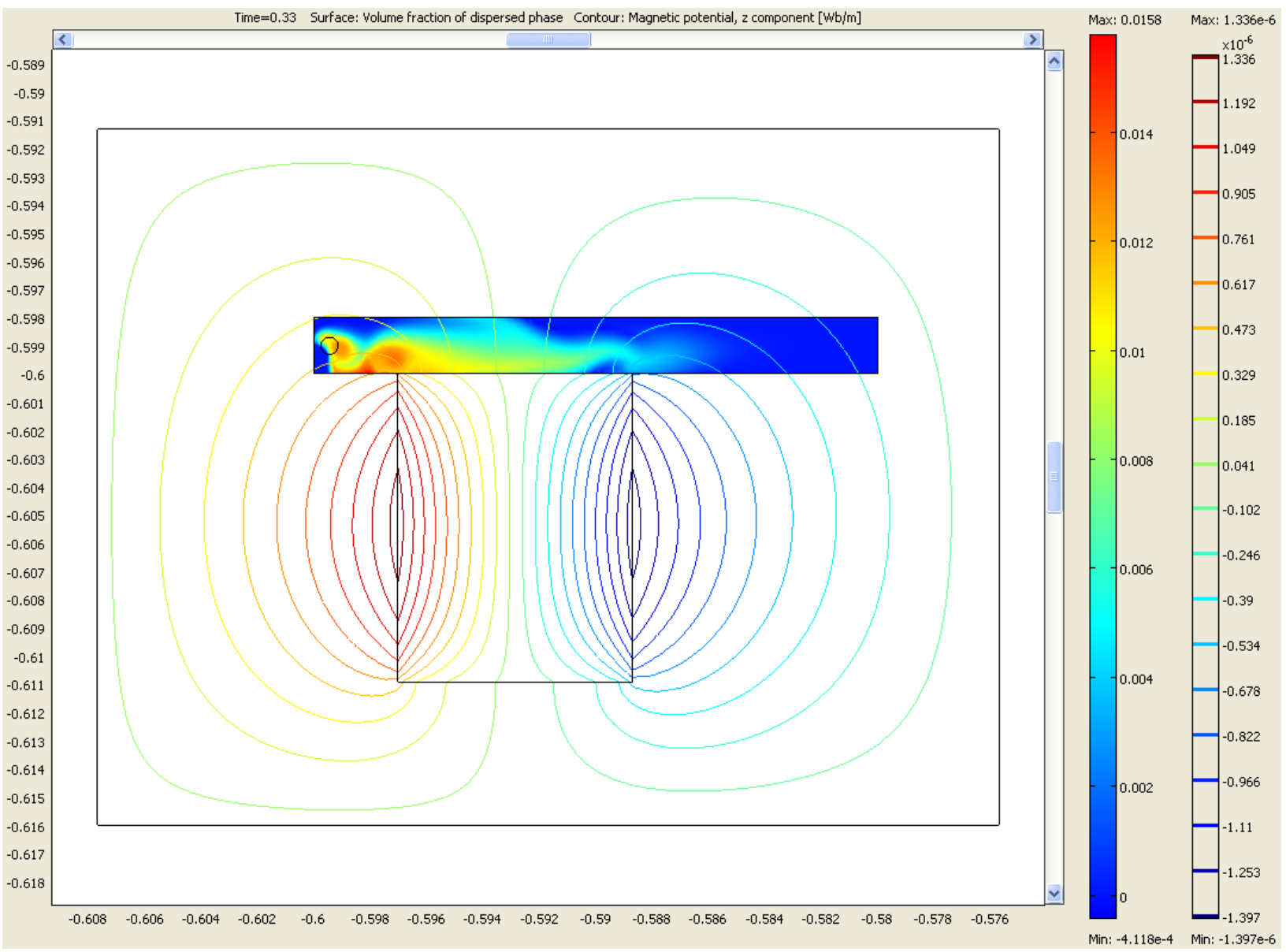

Figure 67: The Volume Fraction Surface Plot and Magnetic Potential Contour Plot for a Reversed Orientation Mixture Model with an Imposed Magnetic Field of 1 T

The volume fraction plots for the model with an increased magnetic field can be seen in Figure 68 through 70 . 


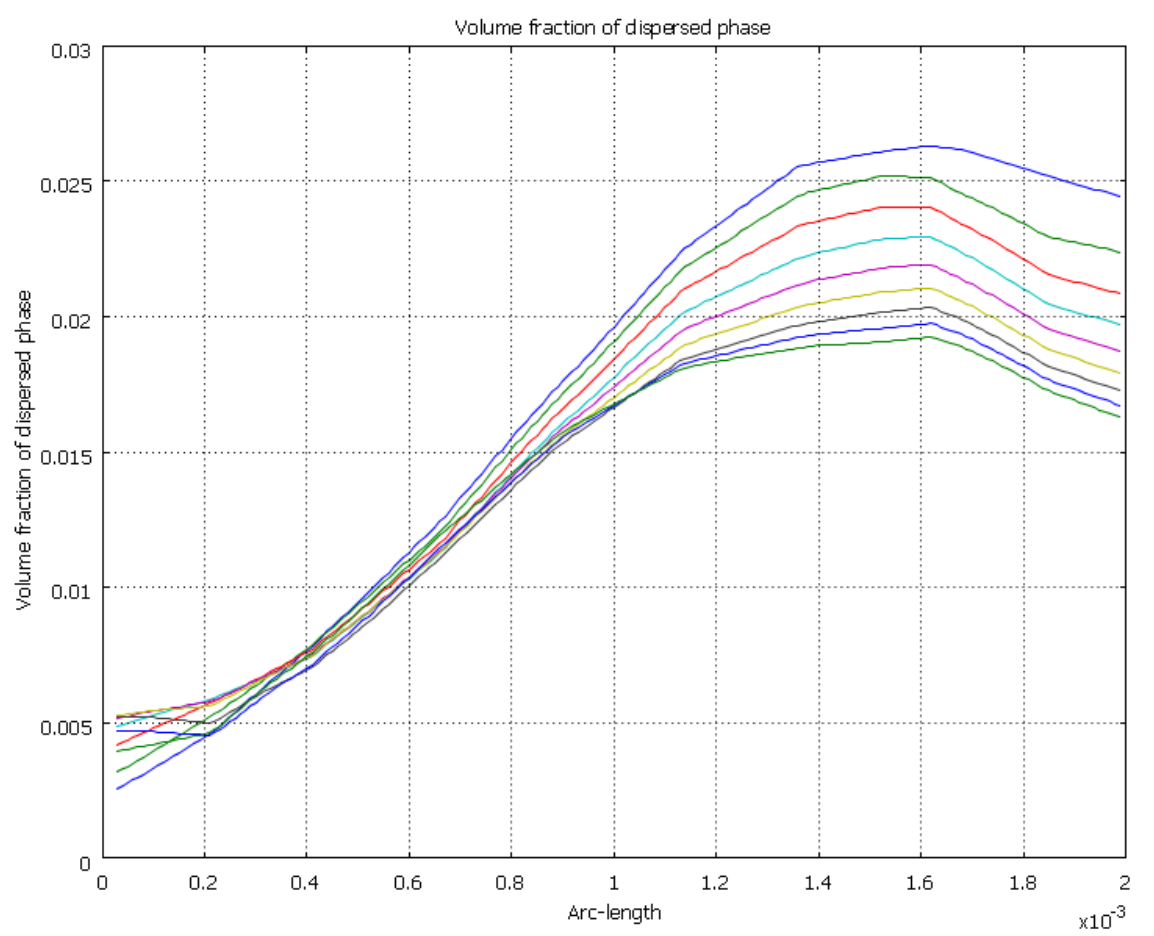

Figure 68: The Volume Fraction of the Mixture Model with the Increased Magnetic Field above the Left Side of the Magnet

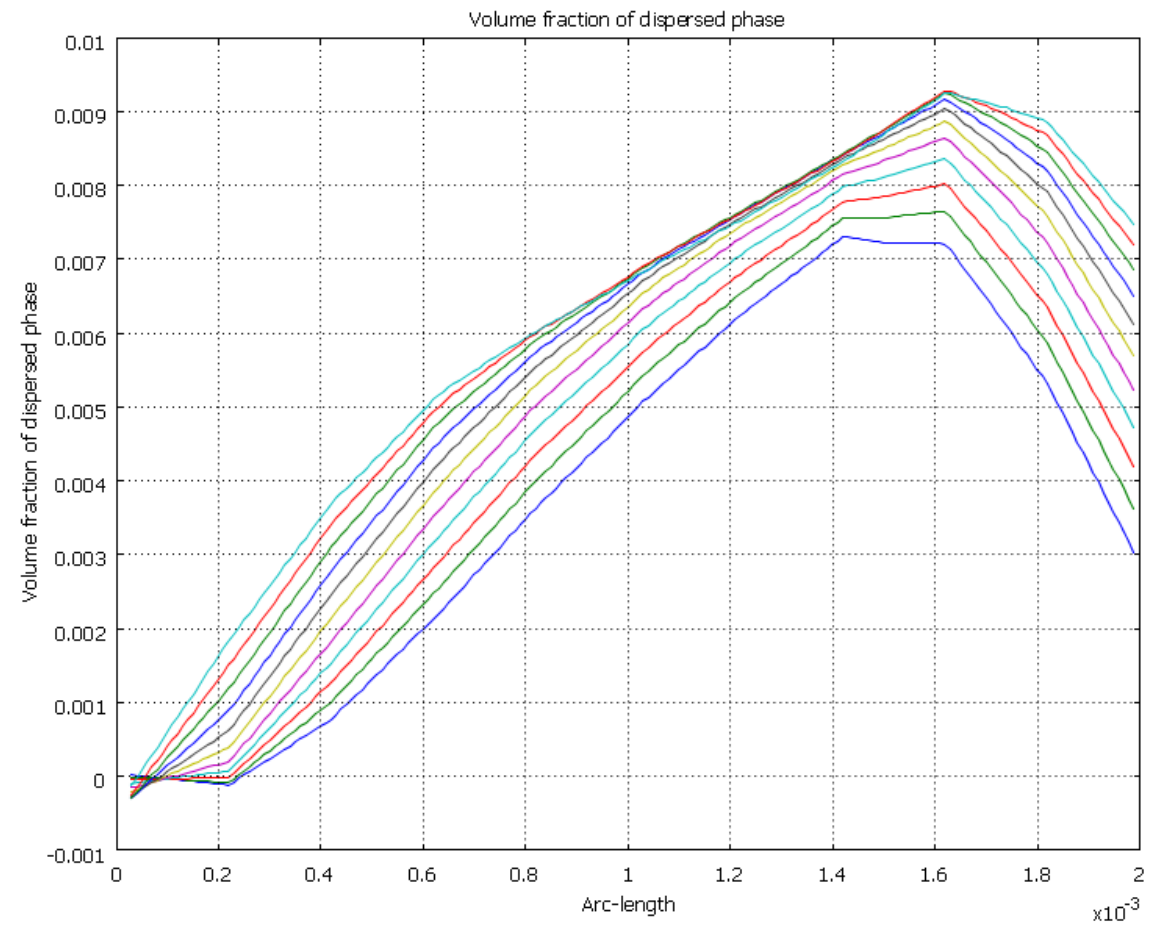

Figure 69: The Volume Fraction of the Mixture Model with the Increased Magnetic Field above the Middle of the Magnet 


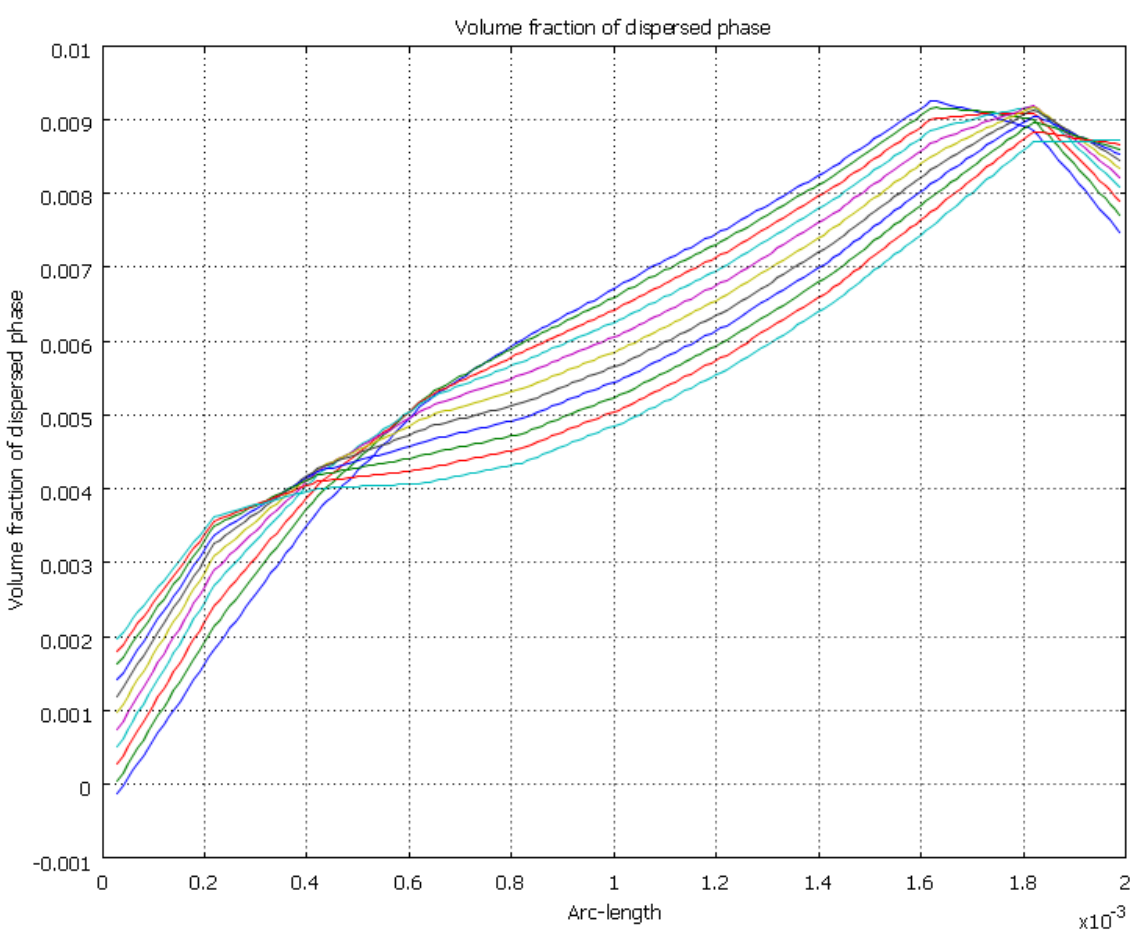

Figure 70: The Volume Fraction of the Mixture Model with the Increased Magnetic Field above the Right Side of the Magnet

Compared to the volume fraction plots for the same model orientation with a magnetic field of $0.5 \mathrm{~T}$, the maximum volume fraction attracted to the magnet has decreased to values comparable to the model orientation where the magnet rests upon the channel. In addition, the slope of the volume fraction lines beneath the right side of the magnet, seen in Figure 70, suggests that the $1 \mathrm{~T}$ magnetic field is insufficient to attract the magnetic particles lost while traveling beneath the middle of the magnet. As a result, a smaller magnetic field is more efficient for the system.

Next, a decrease in the magnetic field of the model to $0.25 \mathrm{~T}$ is implemented, and the results are depicted in Figure 71 through Figure 74. 


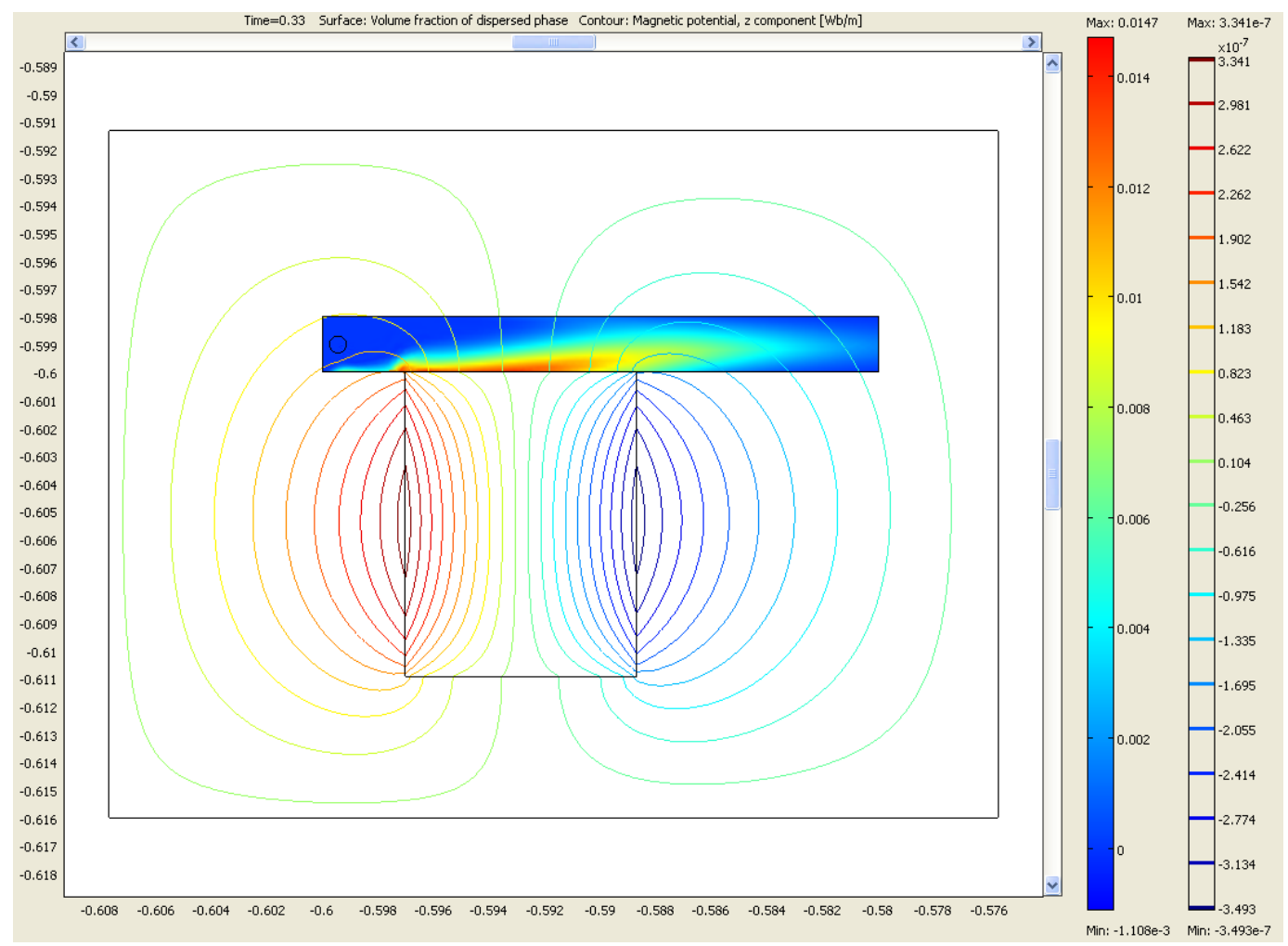

Figure 71: The Volume Fraction Surface Plot and Magnetic Potential Contour for a Model with a Decreased Magnetic Field

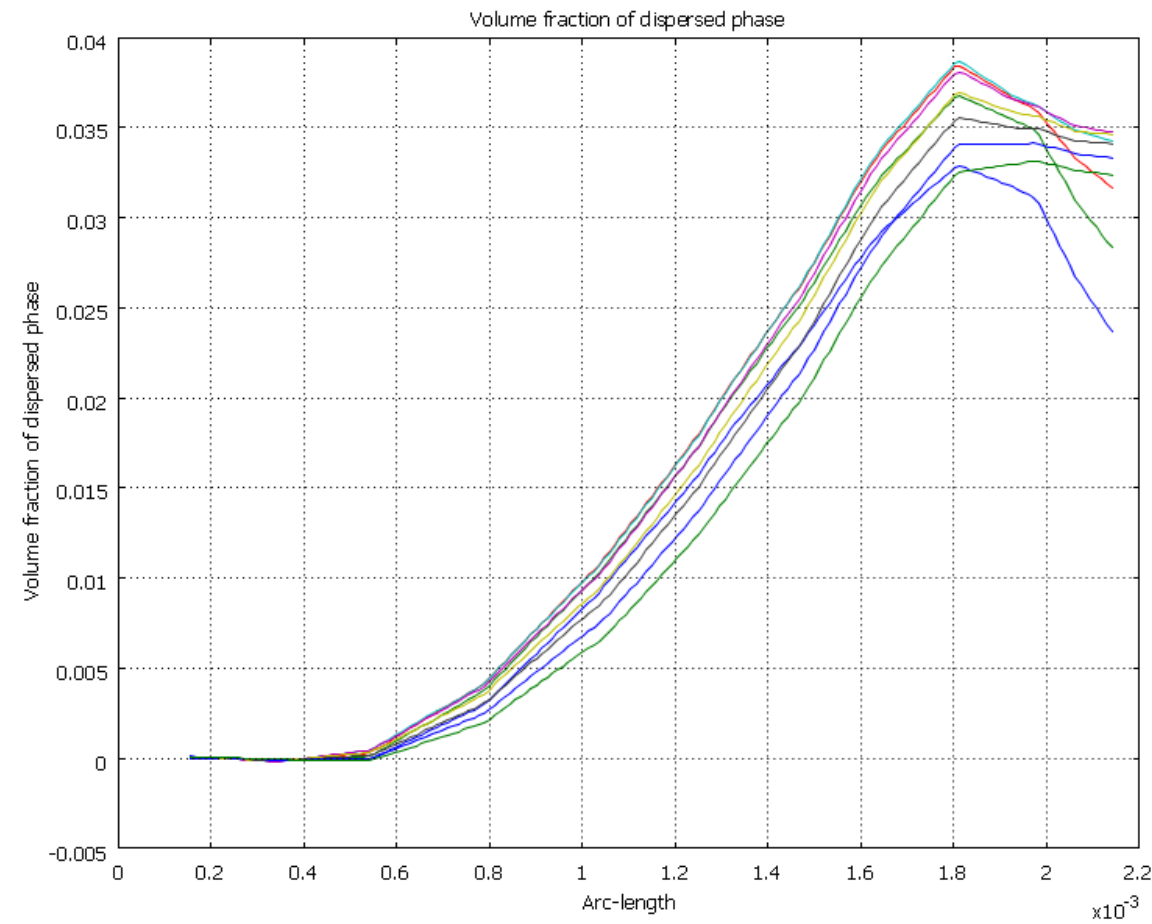

Figure 72: The Volume Fraction of the Mixture Model with the Decreased Magnetic Field above the Left Side of the Magnet 


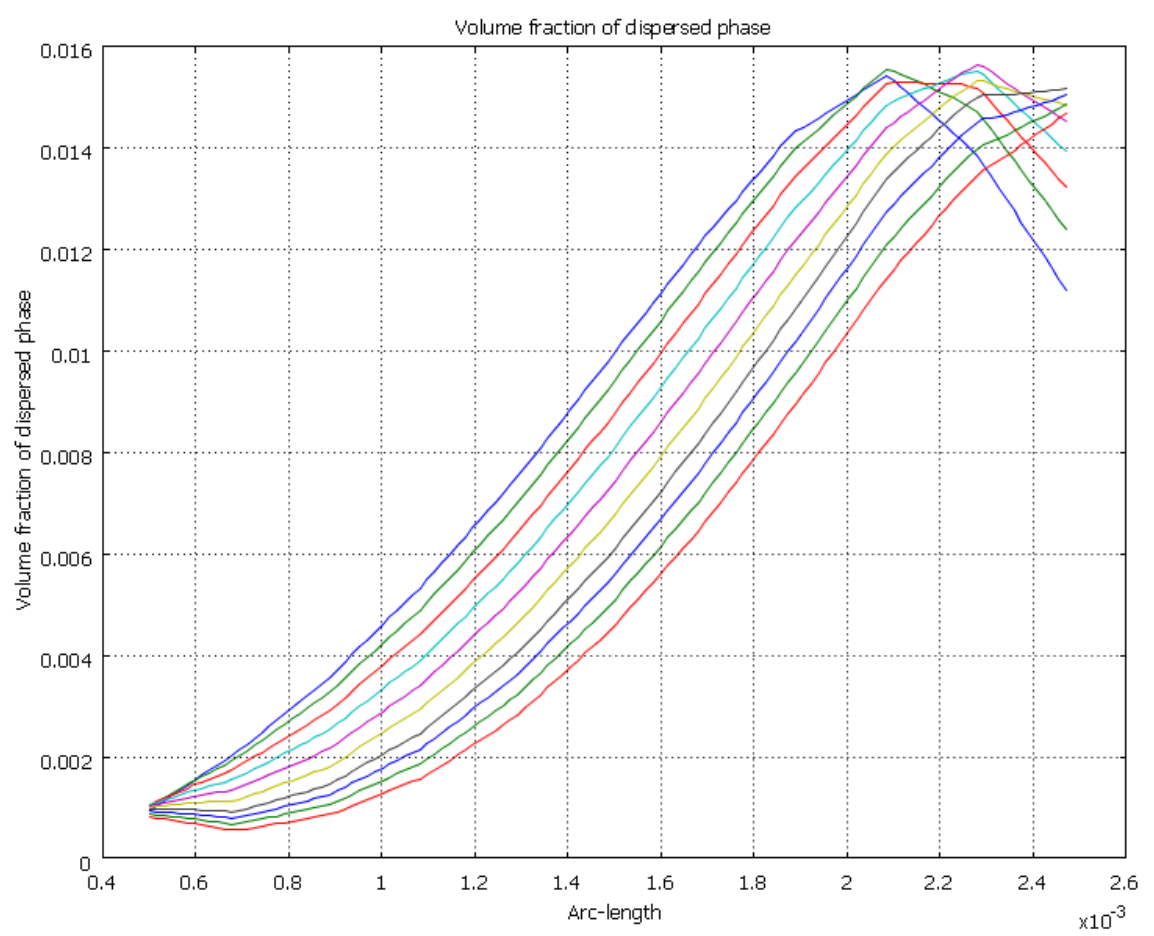

Figure 73: The Volume Fraction of the Mixture Model with the Decreased Magnetic Field above the Middle of the Magnet

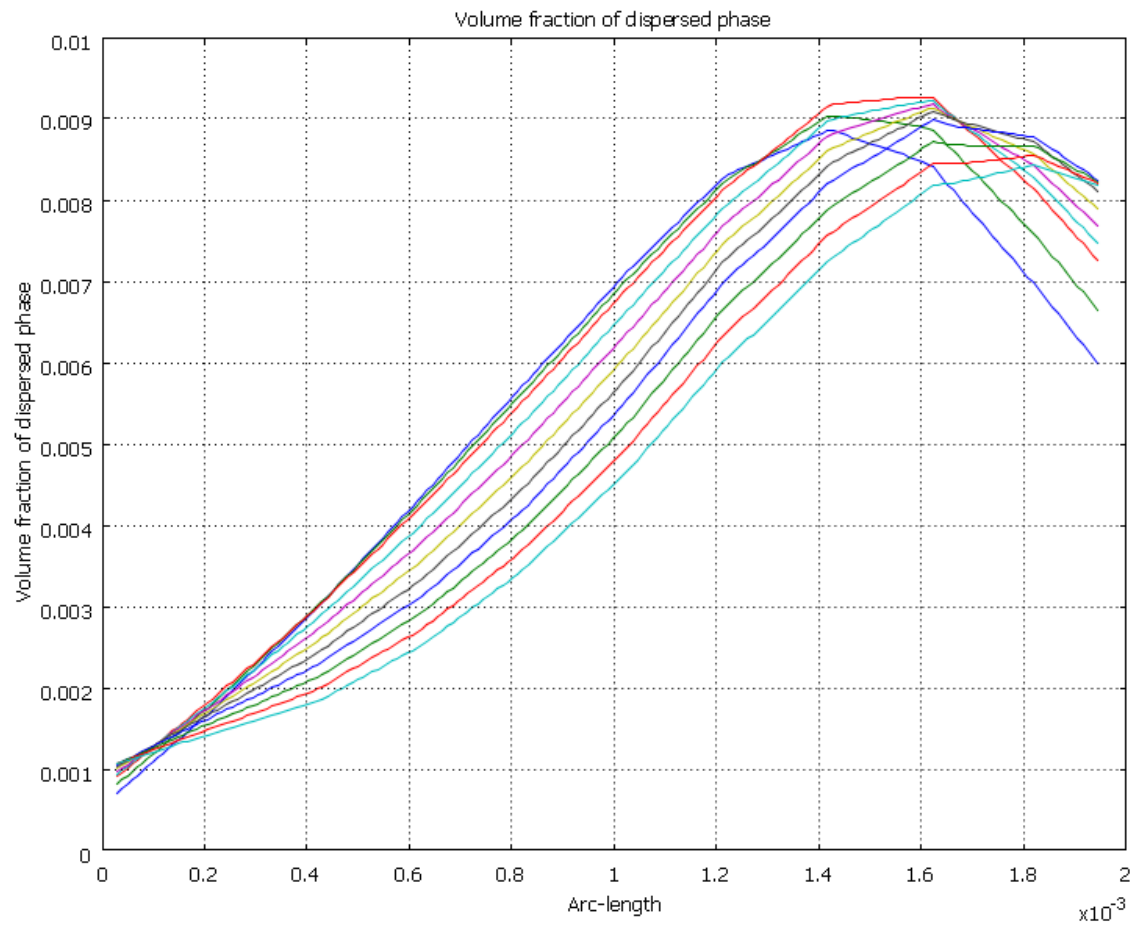

Figure 74: The Volume Fraction of the Mixture Model with the Decreased Magnetic Field above the Right Side of the Magnet 
Comparing these results to a magnetic field of 0.5 T depicted in Figures 64 through 66 , the maximum volume fraction has increased beneath the left side and middle of the magnet but remained about the same above the right side of the magnet. Consequently, the decrease has also increased the volume fraction difference between the bottom and top of the channel, suggesting that attraction of the magnetic particles to the magnet has increased. For the location beneath the left side of the permanent magnet, the increased attraction has resulted in $0.4 \mathrm{e}-3 \mathrm{~m}$ below the top of the channel having 0 volume fraction of the superparamagnetic particles. Below a magnetic field of $0.25 \mathrm{~T}$, the volume fraction for each location will decrease. As a result, a model with a magnetic field of $0.25 \mathrm{~T}$ with an orientation consisting of the channel above the permanent magnet would be optimum for the magnetic trapping system.

To better understand the movement of the superparamagnetic particles in the microfluidic channel, the velocity fields of the Mixture Model when the fluid is stagnant and the inlet pressure is 10 Pa are plotted for the height of the channel beneath the left side of the magnet at three different magnetic field strengths $0.25 \mathrm{~T}, 0.5 \mathrm{~T}$, and $1 \mathrm{~T}$. For these models, the system has an orientation with the permanent magnet located above the microfluidic channel and negligible gravitation effects. These results can be seen in Figures 75 through 77. 

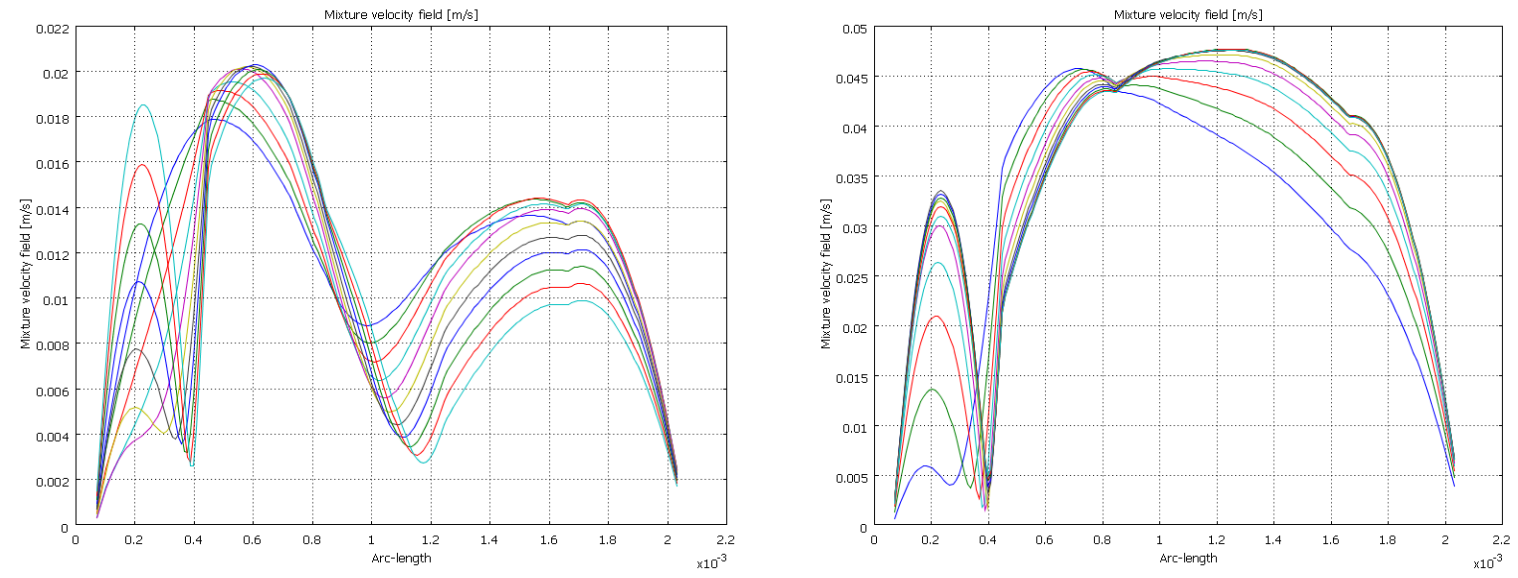

Figure 75: The Velocity Fields for the Channel Height of the System for Stagnant (Left) and Regular (Right) Flow Conditions for a Magnetic Field Strength of $0.25 \mathrm{~T}$
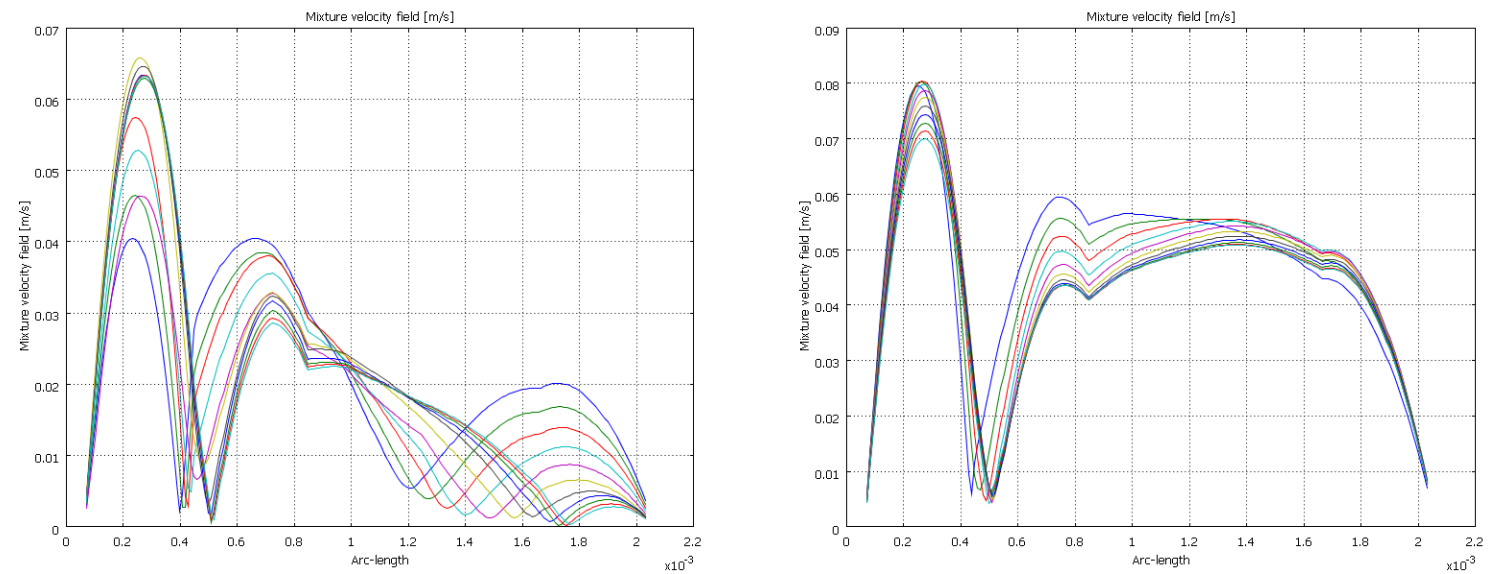

Figure 76: The Velocity Fields for the Channel Height of the System for Stagnant (Left) and Regular (Right) Flow Conditions for a Magnetic Field Strength of $0.5 \mathrm{~T}$
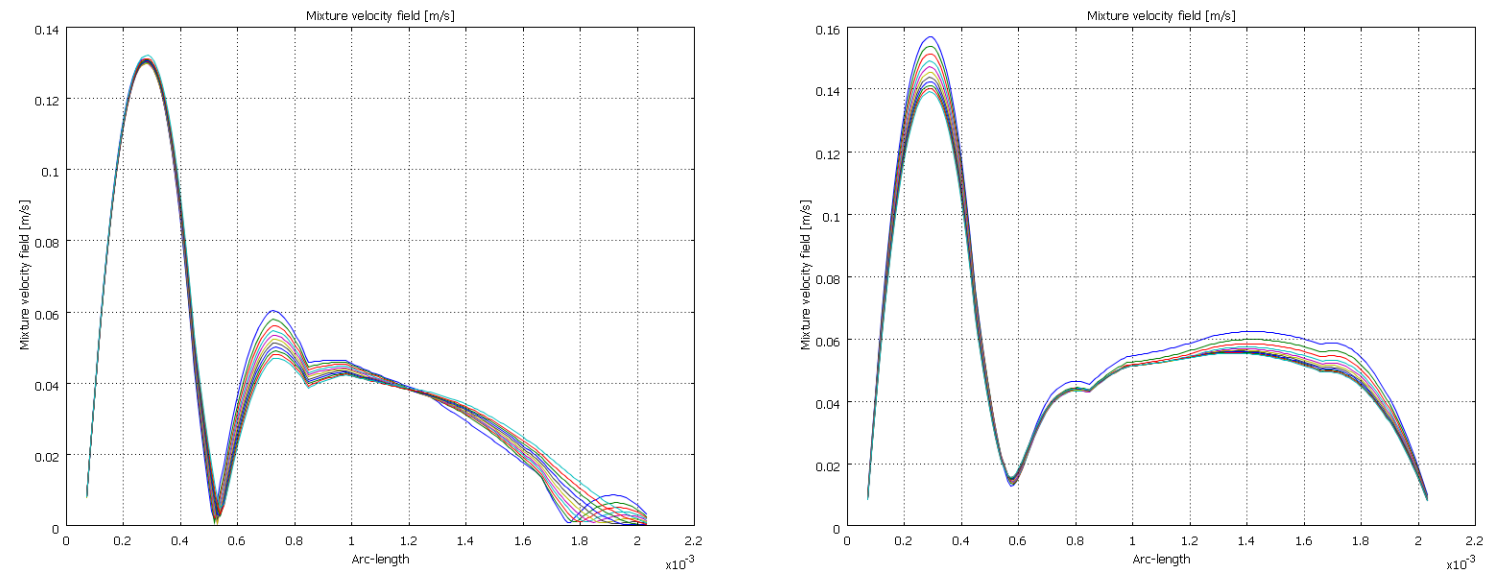

Figure 77: The Velocity Fields for the Channel Height of the System for Stagnant (Left) and Regular (Right) Flow Conditions for a Magnetic Field Strength of $1 \mathrm{~T}$ 
Comparing these results, the velocity fields for the $0.5 \mathrm{~T}$ and $1 \mathrm{~T}$ plots correspond in magnitude and trend. For these plots, the velocity field is greatest at approximately $0.3 \mathrm{~mm}$ beneath the left side of the magnet. In the plots with stagnant fluid, the channels have a velocity of $0 \mathrm{~m} / \mathrm{s}$ at approximately 0.5 $\mathrm{mm}$ beneath the magnet, increase slightly for about $0.1 \mathrm{~mm}$, and then decrease until $0 \mathrm{~m} / \mathrm{s}$ at the bottom wall of the channel. These results indicate that the superparamagnetic particles throughout the height of the channel are affected by the magnetic field; however the greatest effect occurs within 0.8 $\mathrm{mm}$ of the top of the microfluidic channel. In the $0.5 \mathrm{~T}$ and $1 \mathrm{~T}$ plots for an inlet condition of $10 \mathrm{~Pa}$, the magnitude of the velocity has increased and the location of the maximum velocity remains the same. However, the inlet condition results in a partially-parabolic trend starting at $0.6 \mathrm{~mm}$ beneath the top of the microfluidic channel until the bottom of the channel. In the $0.25 \mathrm{~T}$ plots, the particles in the stagnant microfluidic channel are continuously moving through the channel as a result of the magnetic field. The maximum velocity is located $0.6 \mathrm{~mm}$ beneath the magnet. With the $10 \mathrm{~Pa}$ inlet condition, the velocity field follows a similar trend to that of the $0.5 \mathrm{~T}$ and $1 \mathrm{~T}$ model except the velocity for the lower $.16 \mathrm{~mm}$ of the channel is larger than the $0.4 \mathrm{~mm}$ closest to the magnet.

The observed trends in the stagnant velocity plots and volume fractions for the different magnetic fields are most likely the result of particle-particle interactions in the microfluidic channel. In the case of the higher magnetic field strengths, the acquired magnetization in the particles causes repulsion and the smaller volume fractions closest to the magnet. For the $0.25 \mathrm{~T}$ magnetic field, the relative magnetization of the particles appears to be smaller than their attraction toward the magnet, resulting in a more continuous velocity field and larger volume fraction closest to the magnet.

\subsection{Conclusion}

$\mathrm{COMSOL}^{\circledR}$ has been successfully used to provide an environment to help understand and unleash the application of superparamagnetic particles for bio-separation in microfluidic devices 
through the Mixture Model presented in the preceding sections. The Mixture Model successfully accomplished all three of the objectives identified in Section 1.7. It depicts the movement of the superparamagnetic particle fluid by illustrating the volume fraction of this fluid over time as seen in Figures 47 to 55 . Through this model, the attractive effects on the particles are monitored, providing important information for experimental research. The model is quantified through the volume fraction of the superparamagnetic particle fluid and is plotted over the length or width of the channel as seen in several figures including Figure 56 through Post-Processing tools. With the Mixture Model, parameters can be changed and the resulting volume fraction of the superparamagnetic particle fluid for any location can be displayed and compared to optimize the system design of a magnetic trapping system, achieving the third objective. By changing the orientation and magnetic field of the system and including inertial effects, a trapping system with a fluidic channel above a permanent magnet and a magnetic field of $0.25 \mathrm{~T}$ has been identified as the optimum design for a permanent magnet with a height of $1.1 \mathrm{~cm}$ and a width of $8 \mathrm{~mm}$, channel with dimensions of $2 \mathrm{~mm}$ by $2 \mathrm{~cm}$, an inlet within the fluidic channel with a radius of $3.0 \mathrm{e}-4 \mathrm{~m}$, and an inlet pressure of $10 \mathrm{~Pa}$.

The first model or the Particle Motion Model accomplishes only the first objective presented in Section 1.7 by illustrating the movement of a single superparamagnetic bead in a magnetic field as seen in Figures 42 through 45 . Unfortunately, this particle does not travel far before inverted mesh elements prevent complete solution; in fact, the model only solved long enough for magnetic effects to be taken into account. Although this model did not solve for very long, it provides detailed magnetic force analysis on individual superparamagnetic particles.

\subsection{Future Directions}

Validation of the models should be performed to determine their accuracy. Such validation can be done experimentally in vitro through the use of flow cytometry. Flow cytometry identifies and counts 
the number of particles passing a certain location or removed from the outlet of microfluidic channel using the particle's fluorescence, a laser that can excite the particle, and an optical detection system. For detecting the number of trapped particles, the magnetic separator system can be set up as a flow cytometry system with a laser pointing towards a specified location within the microfluidic channel and an incorporated detection system for monitoring the number of particles that pass that location. In addition, a commercial flow cytometer can be used to count the number of superparamagnetic particles collected from the outlet of the microfluidic channel when the magnetic field is both present and absent, revealing the number of particles captured and released. These numbers can then be converted to a volume fraction for comparison with the Mixture Model.

In future modeling of superparamagnetic particles, each of the models presented in the preceding sections can be improved. To avoid the inverted mesh elements, the mesh of the Particle Motion Model geometry can be refined or remeshing can be done [33]. Since mesh refinement and its associated solution consume a great deal of memory, the available memory limitations and the Particle Motion Model mesh prevented both mesh refinement and remeshing. Once the inverted mesh elements can be avoided to allow a sufficient solution, the modeling of multiple particles can be conducted for an understanding of particle interaction.

For more realistic simulations, three-dimensional modeling would be ideal to emulate the actual system. Three-dimensional models are limited by the $\mathrm{COMSOL}^{\circledR}$ physics modes available to research groups. $\mathrm{COMSOL}^{\circledR} 3.5 \mathrm{a}$ was used for the creation of the models presented in Chapters 4 and 5 , and this version does not have a three-dimensional Magnetostatics mode available, preventing a threedimensional model. Currently, a three-dimensional Magnetostatics mode is not available in any versions of $\mathrm{COMSOL}^{\circledR}$; however, three-dimensional modeling can be pursued using the PDE physics mode and implementing all of the necessary magnetostatics equations for the model. 
As discussed in Section 1.5, most of the previously published $\mathrm{COMSOL}^{\circledR}$ models for superparamagnetic particles are similar with the frequent coupling of the Incompressible Navier-Stokes and Magnetostatics modes. Future models should investigate the use of other physics modes such as the Phase Field Method for Two-Phase Flow [38]. This model simulates the flow of two immiscible fluids while taking into consideration surface tension and gravity. For this model, the superparamagnetic particles would be simulated as individual incredibly viscous fluids and, as a result, would enable modeling of individual particles. While calculating a solution, $\mathrm{COMSOL}^{\circledR}$ consumes a great deal of computer memory and can take several hours to solve. By using a nonconservative property setting, the model can solve quicker but will result in the loss of mass of the particle as seen in Figure 78 through Figure 83.
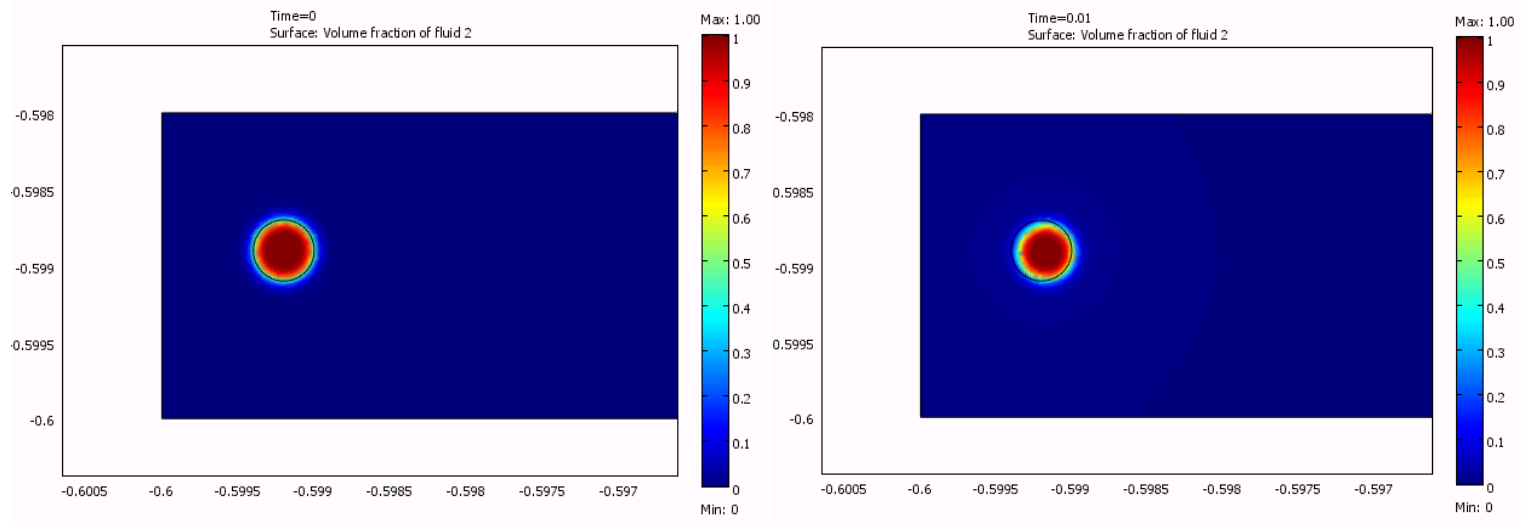

Figure 78: Particle Motion in the Particle Phase Field Mode at $0 \mathrm{~s}$ (Left) and $0.01 \mathrm{~s}$ (Right) 

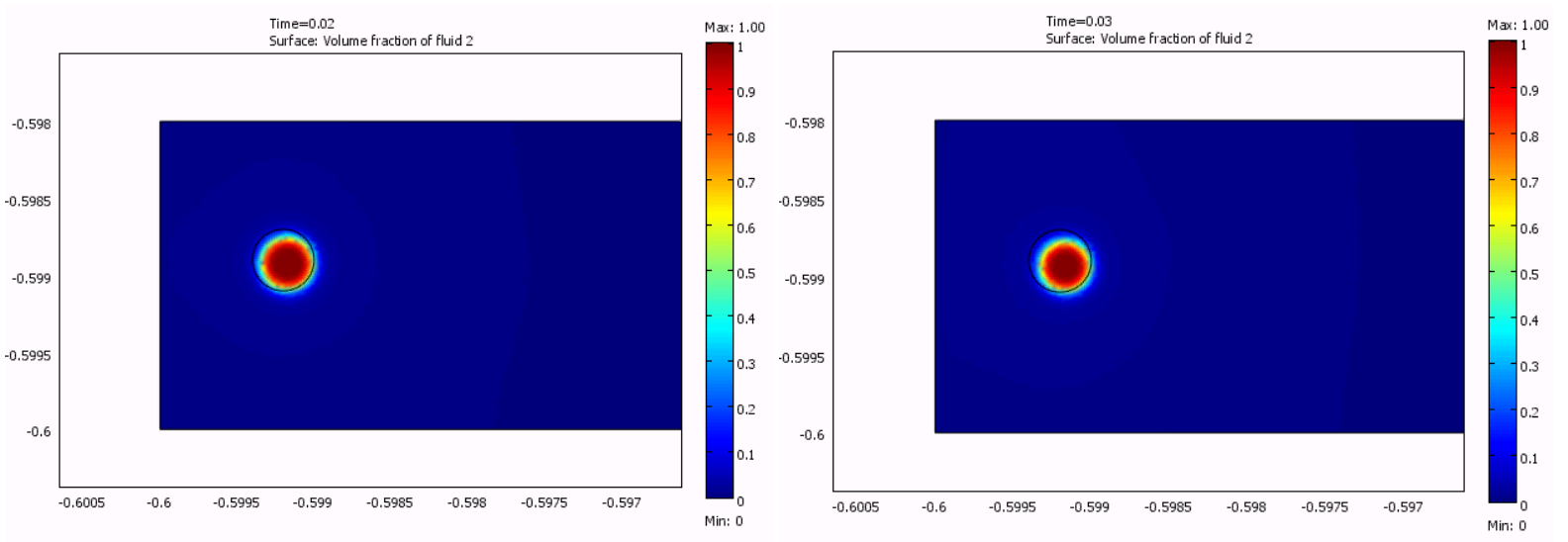

Figure 79: Particle Motion in the Particle Phase Field Mode at $0.02 \mathrm{~s}$ (Left) and $0.03 \mathrm{~s}$ (Right)
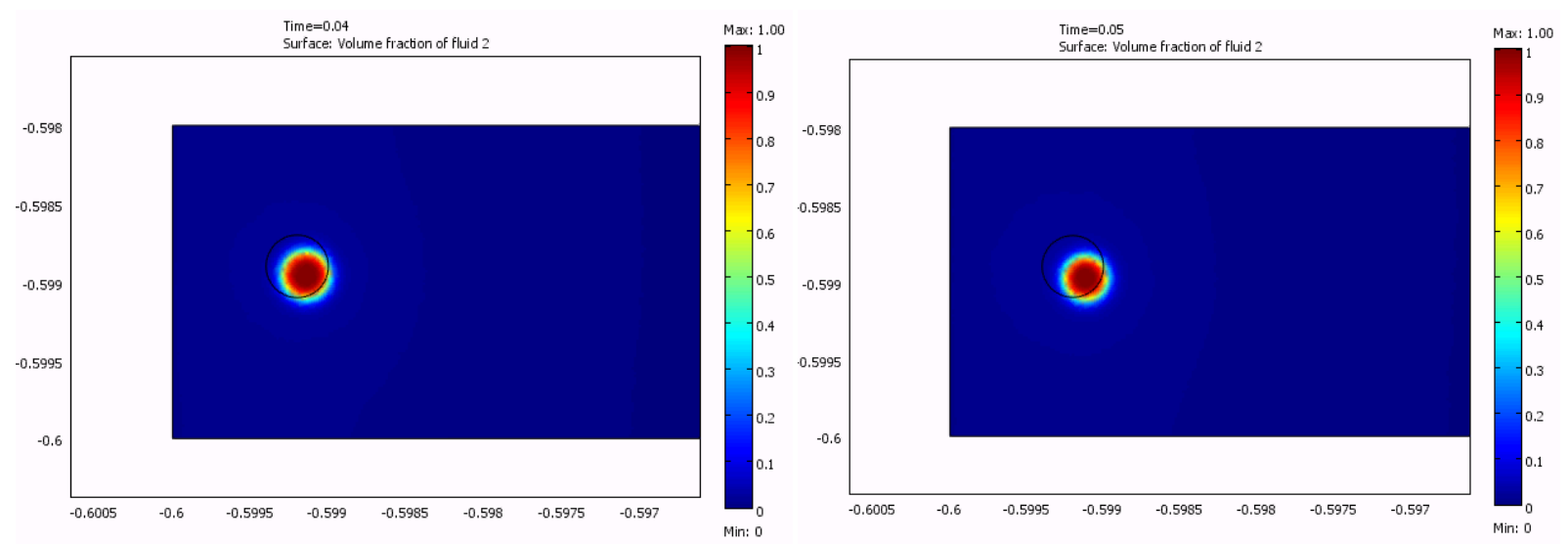

Figure 80: Particle Motion in the Particle Phase Field Mode at $0.04 \mathrm{~s}$ (Left) and $0.05 \mathrm{~s}$ (Right)
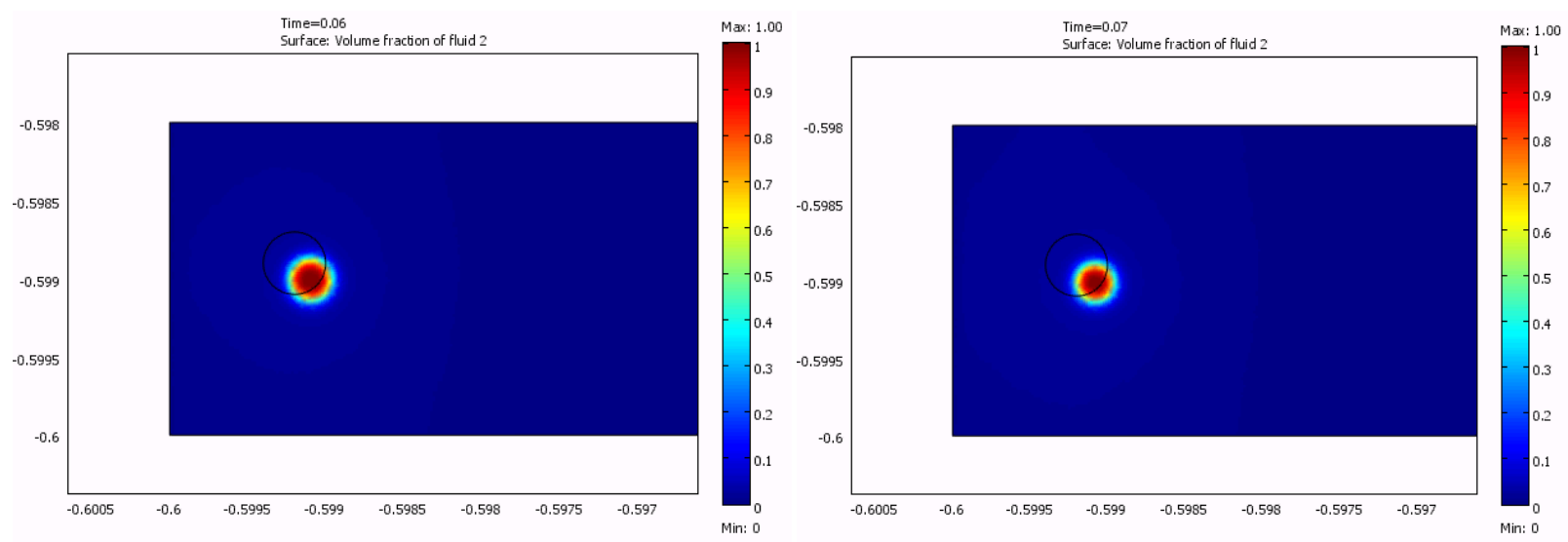

Figure 81: Particle Motion in the Particle Phase Field Mode at $0.06 \mathrm{~s}$ (Left) and $0.07 \mathrm{~s}$ (Right) 

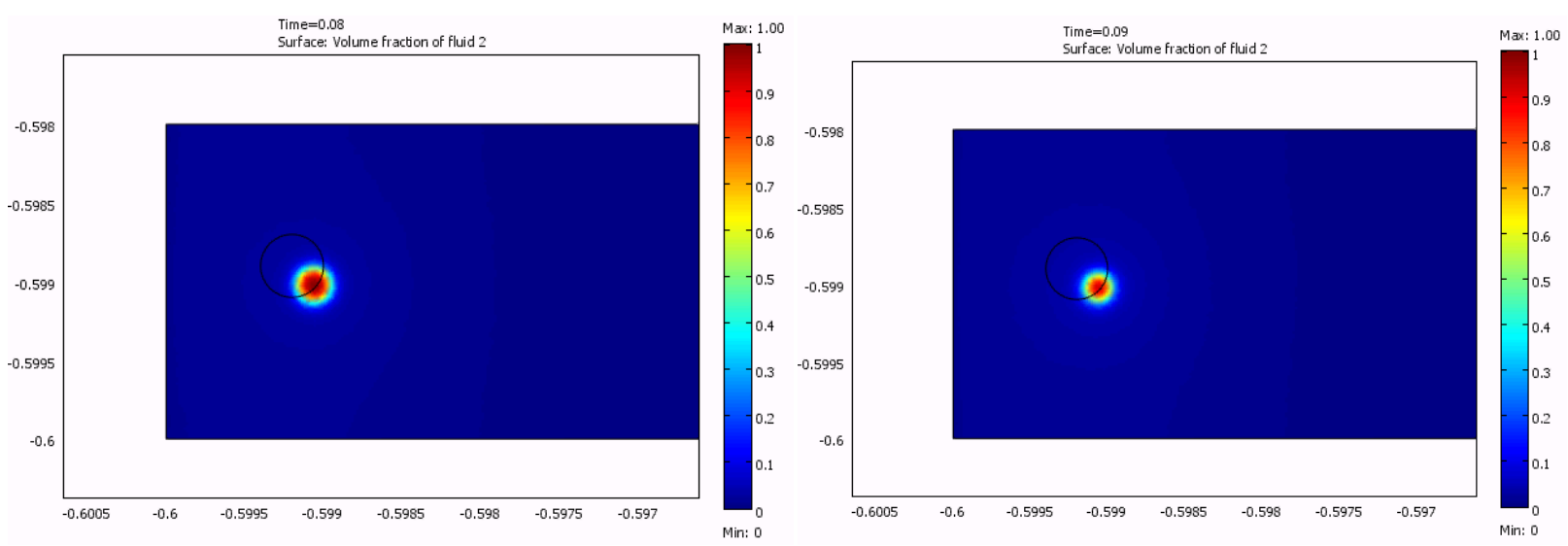

Figure 82: Particle Motion in the Particle Phase Field Mode at $0.08 \mathrm{~s}$ (Left) and $0.09 \mathrm{~s}$ (Right)
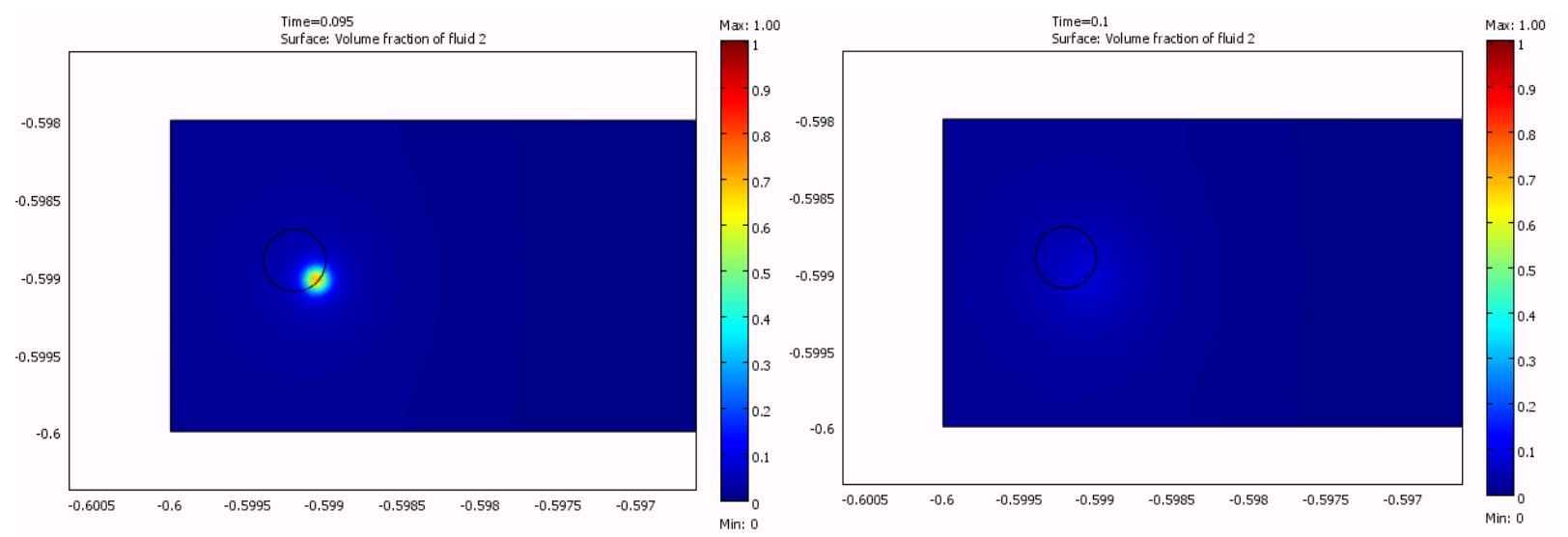

Figure 83: Particle Motion in the Particle Phase Field Mode at $0.095 \mathrm{~s}$ (Left) and $0.1 \mathrm{~s}$ (Right) $\mathrm{COMSOL}^{\circledR}$ offers the opportunity to model superparamagnetic particles prior to experimentation. As this section has discussed, future work in such modeling will be limited by the computer memory and $\mathrm{COMSOL}^{\circledR}$ version available. 


\section{References}

1. Bahadur, D, \& Giri, J. (2003). Biomaterials and magnetism. Sadhana, 28(3), 629-656.

2. Prasad, N.K., Bahadur, D., Vasseur, S., \& Duguet, E. (2008). Biomedical applications of magnetic nanoparticles. In C. Lukehart (Ed.), Nanomaterials: inorganic and bioinorganic perspectives (pp. 15-30). West Sussex, United Kingdom: John Wiley \& Sons, Ltd.

3. Pankhurst, Q.A., Connolly, J., Jones, S.K., \& Dobson, J. (2003). Applications of magnetic nanoparticles in biomedicine. Journal of Physics D: Applied Physics, 36, R167-R181.

4. Thiesen, B., \& Jordan, A,. (2008). Clinical applications of magnetic nanoparticles for hyperthermia. International Journal of Hyperthermia, 24(6), 467-474.

5. Brigger, I., Dubernet, C., \& Couvreur, P. (2002). Nanoparticles in cancer therapy and diagnosis. Advanced Drug Delivery Reviews, 54, 631-651.

6. Yu, Y., \& Sun, D. (2010). Superparamagnetic iron oxide nanoparticle 'theranostics' for multimodality tumor imaging, gene delivery, targeted drug, and prodrug delivery. Expert Reviews: Clinical Pharmacology, 3(1), 117-130.

7. Lee, J.E., Lee, N., Kim, H., Kim, J., \& Choi, S.H. (2009). Uniform mesoporous dye-doped silica nanoparticles decorated with multiple magnetite nanocrystals for simultaneous enhanced magnetic resonance imaging, fluorescence imaging, and drug delivery. Journal of American Chemistry Society, 132, 552-557.

8. Parham, N.J., Picard, F.J., Peytavi, R., Gagnon, M., \& Seyrig, G. (2007). Specific magnetic beadbased capture of genomic DNA from clinical samples: application to the detection of Group B streptococci vaginal/anal swabs. Clinical Chemistry, 53(9), 1570-1576. 
9. Chen, G.D., Alberts, C.J., Rodriguez, W., \& Toner, M. (2010). Concentration and purification of human immunodeficiency virus type 1 virions by microfluidic separation of superparamagnetic nanoparticles. Analytical Chemistry, 82(2), 723-728.

10. Magnetic drug targeting in cancer therapy. (2008). COMSOL Multiphysics ${ }^{\circledR}, 1-15$. COMSOL. $^{2}$

11. Veeramachaneni, U.K., \& Carroll, R.L. (2007). Magnetic particle motion in a gradient field. Proceedings of the COMSOL conference (pp. 1-5). Boston: COMSOL.

12. Mayer, M., Fabian, C., \& Brandl, M. (2006). Simulation of accumulation and movement of ferromagnetic microparticles in streaming liquid within a magnetic trap. Proceedings of the COMSOL conference (pp. 24-25). Frankfurt: COMSOL.

13. Atencia, J., \& Beebe, D.J. (2005). Controlled microfluidic interfaces. Nature, 437(29), 648-655.

14. Daw, R., \& Finkelstein, J. (2006). Lab on a chip. Nature, 447(7101), 367.

15. Whitesides, G.M. (2006). The origins and the future of microfluidics. Nature, 442(27), 368-373.

16. Medoro, G., Manaresi, N., Leonardi, A., Atlomare, L., \& Tartagni, M. (2003). A lab-on-a-chip for cell detection and manipulation. IEEE Sensors Journal, 3(3), 317-325.

17. Squires, T.M., \& Quake, S.R. (2005). Microfluidics: fluid physics at the nanoliter scale. Reviews of Modern Physics, 77, 977-1026.

18. Munson, B.R., Young, D.F., Okisshi, T.H., \& Huebsch, W.W. (2009). Fundamentals of fluid mechanics. Hoboken, NJ: John Wiley \& Sons, Inc.

19. Waite, L., \& Fine, J. (2007). Applied biofluid mechanics. New York, NY: McGraw-Hill.

20. Jones, T.B. (1995). Electromechanics of particles. New York, NY: Press Syndicate of the University of Cambridge.

21. Helmenstine, A.M. (2011, March 23). How magnets work. About.com, Retrieved from http://chemistry.about.com/b/2011/03/23/how-magnets-work.htm.

22. Demarest, K.R. (1998). Engineering electromagnetics. Upper Saddle River, NJ: Prentice Hall, Inc. 
23. Knoepfel, H.E. (2000). Magnetic fields. New York, NY: John Wiley \& Sons, Inc.

24. Hayt, H., \& Buck, J.A. (2011). Engineering electromagnetics. New York, NY: McGraw-Hill.

25. Rizzoni, G. (2004). Principles and applications of electrical engineering. New York, NY: McGrawHill.

26. Van Bladel, J. (2007). Electromagnetic fields. Hoboken, NJ: John Wiley \& Sons, Inc.

27. Kip, A.F. (1962). Fundamentals of electricity and magnetism. New York, NY: McGraw-Hill.

28. Bleaney, B.I., \& Bleaney, B. (1957). Electricity and magnetism. London: Oxford University Press.

29. Pamme, N. (2006). Magnetism and microfluidics. Lab Chip, 6, 24-38.

30. Magnasense . (2011). Superparamagnetic nanospheres. Retrieved from http://magnasense.com/index.php?page=superparamagnetic-nanospheres.

31. Shevkoplyas, S.S., Siegel, A.C., Westervelt, R.M., Prentiss, M.G., \& Whitesides, G.M. (2007). The force acting on a superparamagnetic bead due to an applied magnetic field. Lab Chip, 7, 12941302.

32. COMSOL Multiphysics ${ }^{\circledR}$ user's guide. (2008). COMSOL.

33. The moving mesh application mode. (2008). COMSOL Multiphysics ${ }^{\circledR}$ Modeling Guide (pp. 455467). COMSOL.

34. Microfluidics application modes. MEMS module user's guide (pp. 309-384). COMSOL.

35. Structural mechanics. (2008). COMSOL Multiphysics ${ }^{\circledR}$ Modeling Guide (pp. 203-244). COMSOL.

36. Magnetostatics application mode. (2008). COMSOL Multiphysics ${ }^{\circledR}$ Modeling Guide (pp. 97-106). COMSOL.

37. The mixture model. Chemical engineering module user's guide (pp. 206-218). COMSOL.

38. The phase-field method for two-phase flow. Chemical engineering module user's guide (pp. 191194). COMSOL.

39. COMSOL Multiphysics ${ }^{\circledR}$ reference guide. (2008). COMSOL. 


\section{Appendix A}

In this section, the general process for modeling in $\mathrm{COMSOL}^{\circledR}$, and important $\mathrm{COMSOL}^{\circledR}$ information used for the models discussed in Chapter 5 will be presented. The basic steps involved in creating a model in $\mathrm{COMSOL}^{\circledR}$ include:

1. Selecting the applicable physics mode(s) for the desired model

2. Creating or importing the geometry

3. Implementing the Subdomain, Boundary, and Property Settings for the physics mode(s)

4. Applying a mesh to the geometry

5. Solving the model

6. Viewing different characteristics of the solution using post-processing tools

Examples of these processes have been discussed in Chapters 4 and 5, and more information is provided in reference [32]. 


\section{A.1 Classification of COMSOL ${ }^{\circledR}$ Error Messages}

While calculating a solution for a model, a $\mathrm{COMSOL}^{\circledR}$ error message may appear if the model cannot be solved. This error message contains a four number code and a description of the error. Table 7 summaries the error message ranges and associated $\mathrm{COMSOL}^{\circledR}$ category where the error occurred [39].

Table 7: $\mathrm{COMSOL}^{\circledR}$ Error Messages by Category [39]

\begin{tabular}{|c|l|}
\hline Numbers & \multicolumn{1}{|c|}{ Category } \\
\hline $1000-1999$ & Importing Models \\
\hline $2000-2999$ & Geometry Modeling \\
\hline $3000-3999$ & CAD Import \\
\hline $4000-4999$ & Mesh Generation \\
\hline $5000-5999$ & Point, Edge, Boundary, and Subdomain Specification \\
\hline $6000-6999$ & Assembly and Extended Mesh \\
\hline $7000-7999$ & Solvers \\
\hline $8000-8999$ & Postprocessing \\
\hline $9000-9999$ & General \\
\hline
\end{tabular}




\section{A.2 COMSOL ${ }^{\circledR}$ Solvers}

A number of different solvers are available in $\mathrm{COMSOL}^{\circledR}$ to solve PDE-based problems. These solvers are summarized in Table 8 [32].

Table 8: Available Solvers in $\mathrm{COMSOL}^{\circledR}[32]$

\begin{tabular}{|l|l|}
\hline \multicolumn{1}{|c|}{ Solver Type } & \multicolumn{1}{c|}{ Usage } \\
\hline Stationary & For stationary PDE problems \\
\hline Time Dependent & For time-dependent PDE problems \\
\hline Eigenvalue & For eigenvalue PDE problems \\
\hline Parametric & For parameterized sets of stationary PDE problems \\
\hline Stationary Segregated & For stationary multiphysics PDE problems \\
\hline Parametric Segregated & For parameterized sets of stationary multiphysics PDE problems \\
\hline Time Dependent Segregated & For time-dependent multiphysics PDE problems \\
\hline Adaptive & $\begin{array}{l}\text { For stationary PDE problems or linear eigenvalue PDE problems using } \\
\text { adaptive mesh refinement }\end{array}$ \\
\hline Sensitivity & For sensitivity analysis of stationary PDE problems \\
\hline Optimization & For optimization of stationary PDE problems \\
\hline
\end{tabular}

The models in Chapters 4 and 5 used a linear Time-Dependent solver even though

Magnetostatics is a static multiphysics mode. 


\section{A.3 Linear System Solvers in COMSOL ${ }^{\circledR}$}

Table 9 illustrates the various linear system solvers available in $\mathrm{COMSOL}^{\circledR}$ and their usage in models. As discussed in Chapter 4, UMFPACK was used for the Particle Motion and Mixture Models [32].

Table 9: The Linear System Solvers Available in $\mathrm{COMSOL}^{\circledR}[32]$

\begin{tabular}{|l|l|}
\hline \multicolumn{1}{|c|}{ Linear System Solver } & \multicolumn{1}{c|}{ Usage } \\
\hline Direct Solvers & A highly efficient direct solver for nonsymeetric systems \\
\hline Direct (UMFPACK) & $\begin{array}{l}\text { An efficient direct solver for symmetric and nonsymmetric systems. It } \\
\text { uses less memory than UMFPACK. }\end{array}$ \\
\hline Direct(SPOOLES) & $\begin{array}{l}\text { A highly efficient direct solver for symmetric and nonsymmetric systems. } \\
\text { It often uses less memory than UMFPACK. }\end{array}$ \\
\hline Direct(PARDISO) & An out-of-core version of PARDISO that stores the LU-factors on disk. \\
\hline Direct(PARDISO out of core) & An efficient direct solver for symmetric, positive-definite systems \\
\hline Direct Cholesky (TAUCS) & \multicolumn{2}{|l|}{} \\
\hline Iterative Solvers & An iterative solver for nonsymmetric problems \\
\hline GMRES & $\begin{array}{l}\text { An iterative solver for nonsymmetric problems. It can handle more } \\
\text { general preconditioners but also uses more memory than GMRES. }\end{array}$ \\
\hline FGMRES & An iterative solver for symmetric, positive definite problems \\
\hline Conjugate gradients & $\begin{array}{l}\text { An iterative solver for nonsymmetric problems. It uses a fixed amount of } \\
\text { memory independent of the number of iterations. Therefore, it typically } \\
\text { uses less memory than GMRES. }\end{array}$ \\
\hline BiCGStab & An iterative solver for elliptic or parabolic problems \\
\hline
\end{tabular}

A direct solver is useful for models with few degrees of freedom and uses more memory than iterative solvers. 